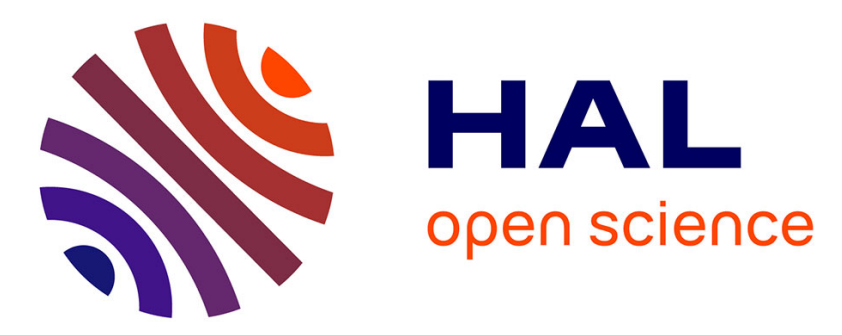

\title{
Gibbs Free Energy of Formation of Chlordecone and Potential Degradation Products: Implications for Remediation Strategies and Environmental Fate
}

\author{
Jan Dolfing, Igor Novak, Alain Archelas, Hervé Macarie
}

\section{- To cite this version:}

Jan Dolfing, Igor Novak, Alain Archelas, Hervé Macarie. Gibbs Free Energy of Formation of Chlordecone and Potential Degradation Products: Implications for Remediation Strategies and Environmental Fate. Environmental Science and Technology, 2012, 46 (15), pp.8131 - 8139. 10.1021/es301165p . hal-01786840

\author{
HAL Id: hal-01786840 \\ https://hal.science/hal-01786840
}

Submitted on 6 May 2018

HAL is a multi-disciplinary open access archive for the deposit and dissemination of scientific research documents, whether they are published or not. The documents may come from teaching and research institutions in France or abroad, or from public or private research centers.
L'archive ouverte pluridisciplinaire HAL, est destinée au dépôt et à la diffusion de documents scientifiques de niveau recherche, publiés ou non, émanant des établissements d'enseignement et de recherche français ou étrangers, des laboratoires publics ou privés. 
Authors' version of the article published in Environ. Sci. \& Technol., 2012, 46, 8131-8139.

The final publication is available at ACS via https://pubs.acs.org/doi/10.1021/es301165p

\title{
Gibbs Free Energy of Formation of Chlordecone and Potential Degradation Products: Implications for Remediation Strategies and Environmental Fate
}

\author{
Jan Dolfing ${ }^{\dagger *}$, Igor Novak ${ }^{\ddagger}$, Alain Archelas ${ }^{\S}$ and Hervé Macarie I*** $^{\text {I* }}$ \\ ${ }^{\dagger}$ School of Civil Engineering \& Geosciences, Newcastle University, Newcastle-upon- \\ Tyne, NE1 7RU, United Kingdom \\ ${ }^{\ddagger}$ Charles Sturt University, Orange, NSW 2800, Australia \\ ${ }^{\S}$ Aix-Marseille Université, CNRS, UMR ISM2, case 332, Faculté des Sciences et \\ Techniques de St Jérome, Avenue Escadrille Normandie Niemen, F-13397 Marseille \\ Cedex 20, France \\ "Aix Marseille Univ, Univ Avignon, CNRS, IRD, IMBE, Marseille, France \\ **IRD, IMBE, Pôle de Recherche Agro-environnemental de la Martinique, Quartier Petit \\ Morne, BP 214, F-97285 Lamentin, France
}

This paper is dedicated in memoriam of Yves-Marie Cabidoche pioneer in the study of the chlordecone problem in the FWI

\begin{abstract}
Chlordecone $\left(\mathrm{C}_{10} \mathrm{Cl}_{10} \mathrm{O}\right.$; CAS number 143-50-0) has been used extensively as an organochlorine insecticide, but is nowadays banned under The Stockholm Convention on Persistent Organic Pollutants (POPs). A search for chlordecone respiring organisms and choosing between reductive versus oxidative remediation tools and strategies to clean up chlordecone polluted environments would benefit from the availability of Gibbs free energy data of chlordecone and its potential dechlorination products. Presently such data are not available. Polycyclic "cage" molecules of which chlordecone is an example contain considerable strain energy. It is not a priory clear how this affects thermodynamic properties of the chlorinated members of this unique class of compounds, and to what extent redox potentials for the halogenated congeners are different from those of other aliphatic and aromatic organohalogens. We have performed $a b$ initio quantum chemical calculations to estimate $\Delta_{\mathrm{f}} H_{\mathrm{m}}{ }^{\mathrm{o}}$ and $\Delta_{\mathrm{f}} G_{\mathrm{m}}{ }^{\mathrm{o}}$ values of chlordecone and selected dechlorination products, and used these data to calculate their Gibbs free energy and redox potential. With redox potentials in the range of 336 to 413 $\mathrm{mV}$ chlordecone has an $E_{\mathrm{o}}{ }^{\prime}$ value similar to that of other organochlorines. The results indicate that there are no thermodynamic reasons why chlordecone respiring or fermenting organisms should not exist.
\end{abstract}

\section{INTRODUCTION}

Chlordecone $\left(\mathrm{C}_{10} \mathrm{Cl}_{10} \mathrm{O}\right.$; CAS number 143-50-0) (Figure 1) is an organochlorine pesticide with all the salient characteristics that have given this class of compounds its deservedly bad reputation. It sorbs strongly to soils, is poorly soluble in water, and highly persistent in the environment; it accumulates in food chains, has potent chronic and acute toxicity, and is a proven carcinogen. ${ }^{1,2}$ Patented as an insecticide in 1952 (US Patent 
$2,616,928)$ it was produced for Allied Chemical Corporation in Hopewell, VA, between 1966 and 1975, mainly for the export to tropical countries where it was used against a wide range of pests, and to Germany where it was converted into Kelevan and used against the Colorado beetle. ${ }^{3,4}$ The production in Hopewell was managed poorly: many of

\begin{tabular}{|c|c|}
\hline IUPAC-Numbering & CAS-Numbering \\
\hline
\end{tabular}

Figure 1. Chlordecone; carbons numbered according to the IUPAC and the CAS nomenclatures.

the laborers developed acute toxicity symptoms, the soils around the plant got severely polluted with chlordecone, and the James River in which the wastewater was discharged after passage through the municipal sewage treatment works had to be closed for the harvest of shellfish and finfish for a period of 18 years. ${ }^{5-7}$ The plants in Hopewell were closed in 1975, and manufacturing and use of chlordecone was forbidden in the US from 1977 onwards. ${ }^{3,4}$ Production of chlordecone moved to Brazil. The active agent was exported from Brazil to France where it was formulated into Curlone (dust with 5\% w/w chlordecone) until the WHO proposed a worldwide ban on its use. ${ }^{8}$ Chlordecone is now banned under The Stockholm Convention on Persistent Organic Pollutants (POPs), a global treaty under auspices of the United Nations ${ }^{9}$, and classifies as legacy POP with all the negative connotations that the word invokes. ${ }^{10}$ For example, chlordecone has been intensively used on the French islands of Martinique and Guadeloupe between 1972 and 1993 against the banana weevil Cosmopolites sordidus; this has resulted in long term pollution of soils and contamination of waters, aquatic biota and crops. ${ }^{11-15}$ Its slow dissipation from the volcanic soils on the islands is ascribed to leaching via drainage water, and a significant part of the original dose ends up in sediments and fish and crustaceans on the islands. ${ }^{12,14}$ History repeats itself, and consumption of fish and crustacean caught on or nearby some parts of the islands is forbidden.${ }^{14}$ Recent data show that chlordecone is present in the blood of adult men, pregnant women, and newborns in Guadeloupe. ${ }^{16-18}$ Main contributors to chlordecone exposure include seafood, root vegetables, and Cucurbitaceae. ${ }^{19}$ Thus, there is growing political and social pressure to find remediation solutions to the chlordecone problem on the French West Indies (FWI). Bioremediation, alone or in combination with physicochemical approaches, could be one of the options. The high chlorine content of the chlordecone molecule (Figure 1) makes anaerobic dechlorination the most likely mode of initial $\operatorname{attack}^{20}:$ a wide series of different aromatic and aliphatic compound classes are susceptible to reductive dechlorination, including chlorinated ethylenes, PCBs and dioxins. ${ }^{21,22}$ 
After the Hopewell disaster in the 1970s there has been a short-lived flurry of research into the environmental fate of chlordecone including some work on biodegradation, funded by the fines levied on Allied Chemicals. ${ }^{23}$ Orndorff and Colwell have performed experiments under aerobic conditions and detected potential degradation products of chlordecone, but limited to the removal of 1 or 2 chlorine atoms from the carbon backbone. ${ }^{24}$ Experiments conducted under anaerobic conditions ${ }^{25,26}$ were more promising. Schrauzer and $\mathrm{Katz}^{25}$ used vitamin $\mathrm{B}_{12 \mathrm{~S}}$ and showed that this coenzyme can dechlorinate chlordecone and can induce opening of the bishomocubane "cage". Jablonski et al. subsequently showed that a pure culture of acetate-grown Methanosarcina thermophila can convert chlordecone to polar and nonpolar products, and obtained a similar pattern of soluble chlordecone decomposition products with reduced vitamin $\mathrm{B}_{12}$ and reduced coenzyme $\mathrm{F}_{430}$ isolated from $M$. thermophila. ${ }^{26}$ Both cofactors are widely distributed among methanogens ${ }^{27}$, which suggests that anaerobic sludges can be used to degrade chlordecone cometabolically. ${ }^{28}$ A complementary hypothesis is that anaerobic bacteria can actually obtain energy for growth from the reductive dechlorination of chlordecone. So far there are no indications for the existence of chlordecone respiring organisms ${ }^{12}$, but the fact that halorespiring anaerobes exist for a wide variety of aliphatic and aromatic organohalides, including hydrophobic compounds like chlorinated dioxins and $\mathrm{PCBs}^{29}$, makes such a hypothesis seem reasonable.

A search for chlordecone degrading micro-organisms and other bioremediation tools to clean up chlordecone polluted soils and sediments would benefit from the availability of Gibbs free energy data of chlordecone and its potential dechlorination and degradation products, or more specifically from Gibbs free energy based calculations that show that reductive dechlorination and mineralization of chlordecone are exergonic processes, which would support the hypothesis that organisms exist that perform these reactions and benefit from it. If reductive dechlorination of chlordecone is not exergonic there is no point in searching for organisms that can perform this reaction; similarly there is no point in testing treatment technologies that use reducing conditions; rather one would focus on oxidizing treatments. Presently such data are not available. Polycyclic "cage" molecules of which chlordecone is an example (Figure 1), contain considerable strain energy. ${ }^{30,31}$ It is not a priory clear how this affects thermodynamic properties of the chlorinated members of this unique class of compounds, and to what extent redox potentials for the halogenated congeners are different from those of other aliphatic and aromatic organohalogens, which are known to be biodegradable and can serve as electron acceptors in anaerobic environments ${ }^{22}$. We have performed high-level $a b$ initio quantum chemical calculations to estimate molar values of $\Delta_{\mathrm{f}} H_{\mathrm{m}}{ }^{\circ}$ and $\Delta_{\mathrm{f}} G_{\mathrm{m}}{ }^{\circ}$ at $25^{\circ} \mathrm{C}$ for chlordecone and selected potential dechlorination products, and used these data to estimate the energetics of the degradation and dechlorination of chlordecone under a variety of redox conditions.

\section{MATERIALS AND METHODS}

Nomenclature and physical and chemical properties. Chlordecone (MW 490.68) is a tan-white crystalline solid with a melting point of $350{ }^{\circ} \mathrm{C}$. Chlordecone belongs to the bishomocubane family of chemicals. ${ }^{30}$ Its IUPAC name is decachloropentacyclo[5.3.0.0 $\left.0^{2.6} \cdot 0^{3.9} \cdot 0^{4.8}\right]$ decan-5-one; its CAS name is 1,1a,3,3a,4,5,5,5a,5b,6-decachlorooctahydro-1,3,4-metheno-2H-cyclobuta[cd]pentalen-2- 
one. Both nomenclatures have been used in the literature ${ }^{32,33}$ which may create confusion when assigning trivial names for some of the intermediates. In the present work the numbering of the carbons will be according to the IUPAC nomenclature (Figure 1). Registered trade names are Kepone, Curlone, Merex, GC 1189 and ENT16391. Other synonyms include perchloro-1,4-bishomocubanone, decachloroketone, and decachlorooctahydro-1,3,4-metheno-2H-cyclobuta[cd]pentalen-2-one.

Quantum chemical calculation methods. $A b$ initio quantum chemical calculations to estimate $\Delta_{\mathrm{f}} H_{\mathrm{m}}^{\mathrm{o}}$ and $\Delta_{\mathrm{f}} G_{\mathrm{m}}^{\mathrm{o}}$ values of chlordecone and selected dechlorination products were performed with the Gaussian 03 software, Revision E. $1^{34}$ using the composite G3(MP2)/B3LYP method. ${ }^{35}$ This method has a mean absolute deviation (MAD) for $\Delta_{\mathrm{f}} H_{\mathrm{m}}{ }^{\mathrm{o}}(\mathrm{g} ., 298.15 \mathrm{~K})$ in the range of $4-8 \mathrm{~kJ} / \mathrm{mol}^{36}$ This MAD value corresponds to the "chemical accuracy" (4-8 kJ/mol) which is required for calculated enthalpy values to be both useful in resolving discrepancies between experimental data and for predicting new thermodynamic data ${ }^{36}$. The choice of the method was based on the need to achieve chemical accuracy and also on the computational feasibility of obtaining such accuracy for the specific molecules investigated in this work. The results of our calculations have been checked against the latest proposed experimental $\Delta_{\mathrm{f}} H_{\mathrm{m}}^{\mathrm{o}}(\mathrm{g} ., 298.15 \mathrm{~K})$ values for similar sized (in terms of the numbers of non-H atoms), strained, cage hydrocarbons: cubane $(613.0 \pm 9.5 \mathrm{~kJ} / \mathrm{mol})$ and dimethyl 1,4-cubanedicarboxylate $(-115.4 \pm 7.0$ $\mathrm{kJ} / \mathrm{mol})^{37}$. Our calculated G3MP2B3/B3LYP values of 608.0 and $-122.7 \mathrm{~kJ} / \mathrm{mol}$, respectively are in good agreement with experimental values. This is important as the "cage" structure of cubane molecules results in strain energy in the carbon skeleton, manifested through long $\mathrm{CC}$ bonds and $\mathrm{CCC}$ angles that deviate significantly from the tetrahedral $109.5^{\circ}$ value. ${ }^{30}$ However, the 1,4-bishomocubane structure which provides the backbone of the chlordecone molecule contains two five-member rings and has therefore considerably less strain energy (viz. $306.9 \mathrm{~kJ} / \mathrm{mol}$ ) than cubane itself $(695.0 \mathrm{~kJ} / \mathrm{mol}){ }^{31}$ These values compare favorably with the strain energy for the perchlorinated 1,4bishomocubane, $(783.1 \mathrm{~kJ} / \mathrm{mol}){ }^{31}$

The G3(MP2)/B3LYP method yields $\Delta_{\mathrm{f}} G^{\mathrm{o}}{ }_{\mathrm{m}}$ and $\Delta_{\mathrm{f}} H_{\mathrm{m}}^{\mathrm{o}}$ values for the gas phase. For environmental applications data for the aqueous phase are more relevant. We therefore used the DPCM solvation model ${ }^{38}$ and G3(MP2)/B3LYP method to simulate water solvent as implemented in the Gaussian software to calculate $\Delta_{\mathrm{f}} G_{\mathrm{m}}^{\mathrm{o}}$ and $\Delta_{\mathrm{f}} H_{\mathrm{m}}^{\mathrm{o}}$ values for the aqueous solution. In the DPCM model, the solvent (in this case water) was represented as a continuous medium with the dielectric constant corresponding to that of water.

Our computational tool was used not because Gaussian software is easy to use, but because it gives good results for the specific set of molecules studied in this work. The use of the tool is also necessary in view of the fact that the experimental methods applied to related cage hydrocarbons ${ }^{37}$ (for example the parent cubane) still show discrepancies of $9 \mathrm{~kJ} / \mathrm{mol}$ in standard enthalpies. This suggests that computational approach for chlordecones is cheaper, faster and more accurate than experimental methods could be at present.

Gibbs free energy values. The G3(MP2)/B3LYP total molecular and atomic energies: $H^{m o l}, H^{a t}, G^{m o l}, G^{a t}$ (which included necessary corrections for zero-point-energy, 
correction terms for particle motions and for entropy at $298.15 \mathrm{~K}$ ) obtained in the gas phase were converted to $\Delta_{\mathrm{f}} H^{\mathrm{o}} \mathrm{m}$ and $\Delta_{\mathrm{f}} G_{\mathrm{m}}^{\mathrm{o}}$ by using the two expressions below.

$\Delta_{\mathrm{f}} H^{o}(\mathrm{~g})=H^{m o l}-\Sigma\left(v_{i} H_{i}{ }^{a t}\right)+\Sigma\left(v_{i}\left(\Delta_{\mathrm{f}} H^{\mathrm{at}}\right)_{\mathrm{i}}\right) ; \Delta_{\mathrm{f}} G^{o}(\mathrm{~g})=G^{m o l}-\Sigma\left(v_{i} G_{i}{ }^{a t}\right)+\Sigma\left(v_{i}\left(\Delta_{\mathrm{f}} G^{\mathrm{at}}\right)_{\mathrm{i}}\right)$

where $v_{i}$ is the stoichiometric coefficient of element $i,\left(\Delta_{\mathrm{f}} H^{a t}\right)_{\mathrm{i}}$ standard formation enthalpy, $\left(\Delta_{\mathrm{f}} G^{a t}\right)_{\mathrm{i}}$ standard Gibbs free energy of formation of $\mathrm{i}$-th element in the gaseous atomic state. The standard values of thermodynamic functions for carbon, hydrogen, chlorine and oxygen were taken from CODATA database in ref. 39. We have also calculated the appropriate total energies $H^{m o l}, H^{a t}, G^{m o l}, G^{a t}$ in the water solvent which allowed us to deduce the solvation energy correction for gas phase data and thus convert $\Delta_{\mathrm{f}} H^{o}(\mathrm{~g})$ and $\Delta_{\mathrm{f}} G^{o}(\mathrm{~g})$ values to $\Delta_{\mathrm{f}} H^{o}(\mathrm{aq})$ and $\Delta_{\mathrm{f}} G^{o}(\mathrm{aq})$. The exact expressions for $H^{m o l}$, $H^{a t}, G^{m o l}, G^{a t}$ are given in Gaussian G03 manual. ${ }^{34}$

In aqueous solutions the standard state of all solutes is $1 \mathrm{~mol} / \mathrm{kg}$ activity, that of water is the pure liquid. Under environmentally relevant conditions the concentrations of substrates and products is not $1 \mathrm{~mol} / \mathrm{kg}$. This is considered in $\Delta G^{\prime}$ values. For a hypothetical reaction $a \mathrm{~A}+b \mathrm{~B} \rightarrow c \mathrm{C}+d \mathrm{D}, \Delta G^{\prime}$ values are calculated by using the mass equation

$$
\Delta G^{\prime}=\Delta G^{o \prime}+R T \ln \left([\mathrm{C}]^{c}[\mathrm{D}]^{d} /[\mathrm{A}]^{a}[\mathrm{~B}]^{b}\right)
$$

The $\Delta G^{o \prime}$ value is obtained from the $\Delta G^{o}$ value by making the appropriate corrections for $\mathrm{pH}=7 .^{40}$

Redox Potentials. Redox potentials were calculated with the relationship ${ }^{40} E_{\mathrm{o}}{ }^{\prime}=(-$ $\Delta G^{\left.O^{\prime} / 0.193\right)-414 .}$

Product distribution. The regiospecificity of chlordecone dechlorination was calculated from the $\Delta_{\mathrm{f}} G^{o}$ values of chlordecone and its potential monohydro substituted dechlorination product according to the equilibrium equation: chlordecone $+\mathrm{H}_{2} \rightleftharpoons$ monohydrochlordecone $+\mathrm{H}^{+}+\mathrm{Cl}^{-}$.

The calculation is based on the premise that $\Delta G_{\text {dechlor }}=-\mathrm{RT} \ln K_{\text {dechlor }}$, where $\Delta G_{\text {dechlor }}$ is the change in Gibbs free energy of a reductive dechlorination reaction and $K_{\text {dechlor }}$ is the corresponding equilibrium constant, i.e.

$K_{\text {dechlor }}=[$ monohydrochlordecone $]\left[\mathrm{H}^{+}\right]\left[\mathrm{Cl}^{-}\right] /[$Chlordecone $]\left[\mathrm{H}_{2}\right] .{ }^{22}$ Therefore $\left(K_{\text {dechlor }} /\right.$ $[$ monohydrochlordecone $])=\left[\mathrm{H}^{+}\right]\left[\mathrm{Cl}^{-}\right] /[$Chlordecone $]\left[\mathrm{H}_{2}\right]$. Thus at a given temperature thermodynamic equilibrium exists between chlordecone and each of its four monohydrochlordecone dechlorination products. Therefore, for each individual monohydrochlordecone the ratio of its concentration over the sum of the concentrations of all four monohydrochlordecones equals the ratio of equilibrium constant with chlordecone over the sum of the equilibrium constants of all four monohydrochlordecones with chlordecone, i.e. for example $M_{l} / M_{\text {total }}=$ $\left(K_{1} /\left(K_{1}+K_{2}+K_{3}+K_{4}\right)\right.$ where $M_{1} / M_{\text {total }}$ is the fraction of the products represented by product $M_{l}{ }^{22}$ 
Henry coefficient. The Henry coefficient $(H)$ in units of atm.L/mol was calculated via the formula $\Delta_{\mathrm{f}} G_{\text {aq }}^{o}=\Delta_{\mathrm{f}} G^{o}$ gas $+R T \ln H$ where $R$ is the universal gas constant (8.314 $\mathrm{J} / \mathrm{K}$.mol) and $T$ is temperature in $\mathrm{K}$. The calculations were made for the standard states of $1 \mathrm{M}$ aqueous concentration and $1 \mathrm{~atm}$ gas pressure, both at $298.15 \mathrm{~K}$.

\section{RESULTS AND DISCUSSION}

The route from chlordecone (perchloro-1,4-bishomocubanone) to its fully dechlorinated product (1,4-bishomocubanone) by sequential dechlorination (removal of $1 \mathrm{Cl}$ at each step without breaking of the carbon structure) encompasses 484 potential intermediates: 92 mesomers plus 196 chirally active compounds (Table 1).

Table 1. Chlordecone and Its Dechlorination Products ${ }^{a}$

\begin{tabular}{|c|c|c|c|c|c|c|c|c|c|c|c|c|}
\hline dechlorination & -0 & -1 & -2 & -3 & -4 & -5 & -6 & -7 & -8 & -9 & -10 & $\Sigma$ \\
& $\mathrm{Cl}$ & $\mathrm{Cl}$ & $\mathrm{Cl}$ & $\mathrm{Cl}$ & $\mathrm{Cl}$ & $\mathrm{Cl}$ & $\mathrm{Cl}$ & $\mathrm{Cl}$ & $\mathrm{Cl}$ & $\mathrm{Cl}$ & $\mathrm{Cl}$ & \\
\hline $\begin{array}{c}\text { meso } \\
\text { compounds }\end{array}$ & 1 & 3 & 7 & 12 & 15 & 18 & 15 & 12 & 7 & 3 & 1 & 94 \\
\hline $\begin{array}{c}\text { enantiomers } \\
(\mathrm{x} 2)\end{array}$ & 0 & $\begin{array}{c}1 \\
(2)\end{array}$ & $\begin{array}{c}9 \\
(18)\end{array}$ & $\begin{array}{c}24 \\
(48)\end{array}$ & $\begin{array}{c}38 \\
(76)\end{array}$ & $\begin{array}{c}52 \\
(104)\end{array}$ & $\begin{array}{c}38 \\
(76)\end{array}$ & $\begin{array}{c}24 \\
(48)\end{array}$ & $\begin{array}{c}9 \\
(18)\end{array}$ & $\begin{array}{c}1 \\
(2)\end{array}$ & 0 & $\begin{array}{c}196 \\
(392)\end{array}$ \\
\hline $\begin{array}{c}\text { total without } \\
\text { enantiomers }\end{array}$ & 1 & 4 & 16 & 36 & 53 & 70 & 53 & 36 & 16 & 4 & 1 & 290 \\
\hline $\begin{array}{c}\text { total with } \\
\text { enantiomers } \\
\text { included }\end{array}$ & 1 & 5 & 25 & 60 & 91 & 122 & 91 & 60 & 25 & 5 & 1 & 486 \\
\hline
\end{tabular}

${ }^{a}$ total: 486 congeners, of which 94 are meso compounds and 196 are chiral compounds. Congeners that potentially have a different Gibbs free energy of formation: 290 (94 + 196).

Table SI-1 (Supporting Information) lists all congeners named according to the IUPAC and CAS nomenclatures. In the present manuscript we used a simplified nomenclature based on the chlordecone name using the IUPAC numeration (i.e. the keto group is on carbon 5) in which the position of the hydrogen substituents is indicated (Figure 1). In addition, when one single $\mathrm{H}$ is on carbon 10 it may be necessary to specify the relative stereochemistry (cis or trans) of this $\mathrm{H}$ with respect to another hydrogen positioned on the bishomocubane "cage". For instance the trivial name for compound 258 in Table SI-1 $\left(r-2,6, t-10\right.$ trichloropentacyclo[5.3.0.0 $\left.0^{2,6} \cdot 0^{3,9} \cdot 0^{4,8}\right]$ decan-5-one, IUPAC nomenclature) will be $1, r-3,4,7,8,9, c-10$-heptahydro-chlordecone which means that the hydrogen on carbon 10 is in the cis $(c-10)$ position relative to the $\mathrm{H}$ on carbon 3 used as reference $(r-3)$.

$\Delta_{\mathrm{f}} G^{o}$ and $\Delta_{\mathrm{f}} H^{o}$ values for chlordecone and selected dechlorination products in gas and aqueous phases are presented in Table 2. The selection includes the isomers observed when chlordecone is reduced with vitamin $\mathrm{B}_{12 \mathrm{ss}}{ }^{25}$ The data for the gas phase are in the format preferred by theoretical chemists because quantum calculations produce the most accurate theoretical results for isolated molecules in the gas phase. The data for the aqueous phase are in the format preferred by environmental chemists and microbiologists. A major advantage of the latter way of presenting $\Delta_{\mathrm{f}} G^{o}$ data is also that 
extrapolation to in situ conditions can be easily done by simply substituting the actual concentration for the standard concentration in equation 1 . Hereafter we will focus on the data in the aqueous solution. A more extensive Table, which also lists the appropriate strain energies, dipole moments, $S^{o}$ values and Henry coefficients and includes more compounds, is given in the Supporting Information (Table SI-2).

Table 2. Calculated $\Delta_{\mathrm{f}} G^{0}$ and $\Delta_{\mathrm{f}} H^{0}$ Values $(\mathrm{kJ} / \mathrm{mol})$ of Chlordecone, Chlordecol and Selected Dechlorinated Congeners in the Gas and the Aqueous Phase ${ }^{a}$

\begin{tabular}{|c|c|c|c|c|c|}
\hline compound & $\begin{array}{l}\text { Entry } \\
\text { in Table } \\
\text { SI-1 }\end{array}$ & $\Delta_{\mathbf{f}} G_{\text {gas }}^{0}$ & $\Delta_{\mathbf{f}} H_{\text {gas }}^{\mathrm{o}}$ & $\boldsymbol{\Delta}_{\mathrm{f}} G_{\mathrm{aq}}^{\mathrm{o}}$ & $\boldsymbol{\Delta}_{\mathbf{f}} H_{\mathrm{aq}}^{\mathrm{o}}$ \\
\hline chlordecone & 1 & -73.5 & -225.9 & -92.0 & -244.6 \\
\hline chlordecol & na & -143.5 & -332.8 & -162.3 & -378.0 \\
\hline $\begin{array}{l}\text { 6-hydrochlordecone } \\
\text { 8-hydrochlordecone* } \\
\text { 9-hydrochlordecone } \\
\text { 10-hydrochlordecone }\end{array}$ & $\begin{array}{l}4 \\
5 \\
3 \\
2\end{array}$ & $\begin{array}{l}-57.8 \\
-\mathbf{5 4 . 5} \\
-54.6 \\
\mathbf{- 5 2 . 3}\end{array}$ & $\begin{array}{l}-205.4 \\
-201.6 \\
-201.8 \\
-199.2\end{array}$ & $\begin{array}{l}-76.3 \\
-80.5 \\
-80.1 \\
-79.2\end{array}$ & $\begin{array}{l}-230.0 \\
-227.8 \\
-227.5 \\
-226.0\end{array}$ \\
\hline $\begin{array}{l}\text { 3,7-dihydrochlordecone* } \\
\text { cis-8,10-dihydrochlordecone* }\end{array}$ & $\begin{array}{l}21 \\
\mathbf{1 4}\end{array}$ & $\begin{array}{l}-36.2 \\
-31.7\end{array}$ & $\begin{array}{l}-177.9 \\
-\mathbf{1 7 3 . 3}\end{array}$ & $\begin{array}{l}-68.8 \\
-66.9\end{array}$ & $\begin{array}{l}-210.7 \\
-\mathbf{2 0 9 . 0}\end{array}$ \\
\hline $\begin{array}{l}\text { 3,7,10-trihydrochlordecone* } \\
\text { 8,10,10-trihydrochlordecone* }\end{array}$ & $\begin{array}{l}40 \\
34\end{array}$ & $\begin{array}{l}-13.2 \\
-13.3\end{array}$ & $\begin{array}{l}-149.6 \\
-150.0\end{array}$ & $\begin{array}{l}-53.5 \\
-43.7\end{array}$ & $\begin{array}{l}-190.2 \\
-180.7\end{array}$ \\
\hline $\begin{array}{l}r-3,7,8, t-10 \text {-tetrahydrochlordecone* } \\
\text { 3,7,10,10-tetrahydrochlordecone }\end{array}$ & $\begin{array}{r}100 \\
77\end{array}$ & $\begin{array}{r}20.8 \\
8.8\end{array}$ & $\begin{array}{l}-111.6 \\
-123.5\end{array}$ & $\begin{array}{l}-26.2 \\
-27.4\end{array}$ & $\begin{array}{l}-158.7 \\
-159.9\end{array}$ \\
\hline $3,7,8,10,10$-pentahydrochlordecone* & 134 & 43.5 & -84.8 & 2.5 & -125.9 \\
\hline 2,3,7,8,10,10-hexahydrochlordecone & 188 & 79.1 & -45.1 & 34.9 & -89.2 \\
\hline 2,3,7,8,9,10,10-heptahydrochlordecone & 239 & 106.4 & -14.0 & 64.0 & -56.4 \\
\hline $1,2,3,7,8,9,10,10$-octahydrochlordecone & 276 & 137.1 & 20.4 & 98.9 & -17.7 \\
\hline $\begin{array}{l}\text { 1,2,3,4,6,7,8,9,10-nonahydrochlordecone } \\
1,2,3,6,7,8,9,10,10 \text {-nonahydrochlordecone } \\
1,3,4,6,7,8,9,10,10 \text {-nonahydrochlordecone* } \\
2,3,4,6,7,8,9,10,10 \text {-nonahydrochlordecone }\end{array}$ & $\begin{array}{l}286 \\
288 \\
289 \\
287\end{array}$ & $\begin{array}{l}165.3 \\
168.3 \\
156.2 \\
162.1\end{array}$ & $\begin{array}{l}52.6 \\
55.9 \\
43.3 \\
49.0\end{array}$ & $\begin{array}{l}124.2 \\
131.8 \\
119.4 \\
125.4\end{array}$ & $\begin{array}{r}11.9 \\
19.6 \\
6.8 \\
12.6\end{array}$ \\
\hline decahydrochlordecone & 290 & 203.8 & 94.2 & 171.9 & 62.5 \\
\hline
\end{tabular}

Thermodynamic Data for the Aqueous State. Calculated standard Gibbs free energies of formation $\left(\Delta_{\mathrm{f}} G^{o}\right.$ aq $)$ of chlordecone and its less chlorinated congeners range between -92.0 and $171.9 \mathrm{~kJ} / \mathrm{mol}$ for chlordecone and decahydrochlordecone, respectively. $\Delta_{\mathrm{f}} G^{o}$ decreases with increasing number of chloro substituents (Figure 2). This can be 
rationalized by noting that $\mathrm{C}-\mathrm{H}$ and $\mathrm{C}-\mathrm{Cl}$ bond enthalpies are 414 and $324 \mathrm{~kJ} / \mathrm{mol}$, respectively. ${ }^{41}$ Therefore the replacement of $\mathrm{C}-\mathrm{H}$ by C-Cl bonds leads to the observed effect in $\Delta_{\mathrm{f}} H^{o}$ aq and $\Delta_{\mathrm{f}} G_{\text {aq }}^{o}$ values. This is also observed for other classes of polycyclic halogenated compounds such as $\mathrm{PCBs}^{42}$ and chlorodioxins ${ }^{43}$ but not for chlorinated aliphatics such as ethanes and ethenes. ${ }^{44}$ Considerable variation exists between the thermodynamic values for different congeners with the same degree of chlorination. For the four monochloro congeners for example $\Delta_{\mathrm{f}} G_{\text {aq }}^{o}$ values range between $119.4 \mathrm{~kJ} / \mathrm{mol}$ for the 2-chloro congener (entry 289 in Table SI-1) and $131.8 \mathrm{~kJ} / \mathrm{mol}$ for the 4-chloro congener (entry 288 in Table SI-1) respectively. The extent of the variation may depend on the degree of chlorination. It is for instance higher for the dichloro congeners (78.0 to $107.1 \mathrm{~kJ} / \mathrm{mol}$ ) (Table SI-1, Supporting Information), but lower for the monohydrochlordecones (-76.3 to $-80.5 \mathrm{~kJ} / \mathrm{mol}$ ). The positions of chlorine substituents (topology) in chlordecone isomers clearly affect $\Delta_{\mathrm{f}} G^{o}$ values, but the effect is affected by the degree of chlorination of the congeners. It is thus virtually impossible to devise a simple group contribution method to describe the $\Delta_{\mathrm{f}} G_{\text {aq }}^{o}$ of all 486 chlordecone congeners.

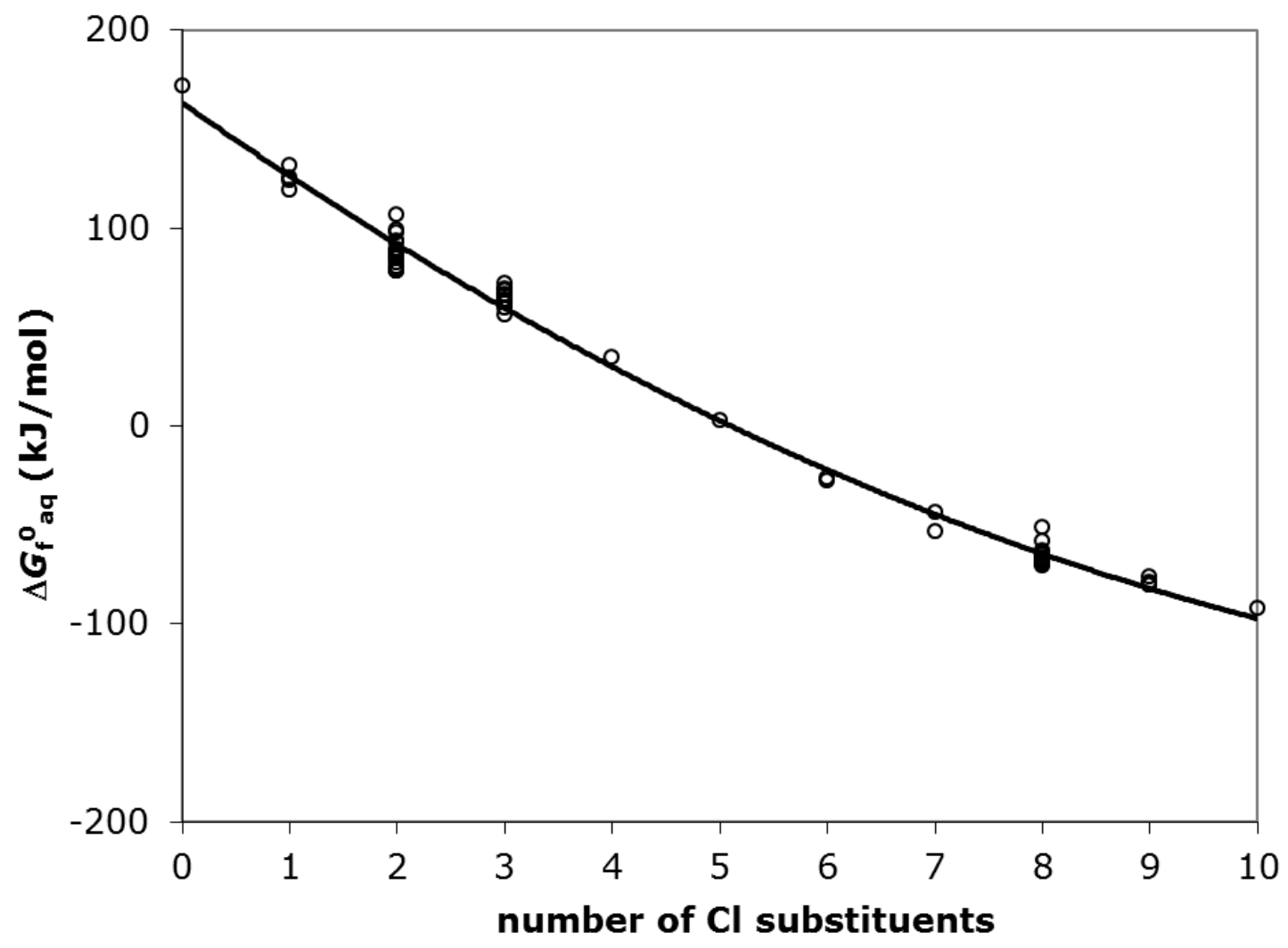

Figure 2. Calculated $\Delta G_{\mathrm{f}}^{\mathrm{o} \text { aq }}$ of chlordecone and less halogenated congeners as affected by the degree of chlorination of the molecule (Data Supporting Information - Table SI-2).

The standard Gibbs free energies and enthalpies of formation correlate linearly with strain energy (Figure SI-1, Supporting Information). This can be expected because strain energy is a major (de)stabilizing influence in an individual molecule. We have also noted 
that isomers which have chlorine substitution at carbon-10 do not follow this correlation, they represent outliers in Figure SI-1 (Supporting Information). This can be expected because these substituents are attached to relatively strain-free five-member rings. The influence of substitution topology i.e. the location of chlorine atoms on individual molecule's thermodynamic functions does not and cannot be expected to follow simple linear correlations. This is due to the presence of nonlinear quantum mechanical effects (e.g. interatomic steric repulsions, bond dipole repulsions between $\mathrm{C}-\mathrm{Cl}$ bonds, distortions of $\mathrm{CCC}$ bond angles and electron densities in different $\mathrm{CC}$ bonds upon vicinal chlorine substitution etc.) which vary with the number and position of chlorine substituents. $\Delta_{\mathrm{f}} G_{\text {aq }}^{o}$ and $\Delta_{\mathrm{f}} H^{o}$ aq are highly correlated $\left(\mathrm{r}^{2}=0.9993\right)$ as are $\Delta_{\mathrm{f}} G_{\text {gas }}^{o}$ and $\Delta_{\mathrm{f}} H^{o}$ gas $\left(\mathrm{r}^{2}=0.9996\right) . \Delta_{\mathrm{f}} G_{\text {aq }}^{o}$ and $\Delta_{\mathrm{f}} G_{\text {gas }}^{o}$ values correlate less well $\left(\mathrm{r}^{2}=0.9914\right)$, and visual inspection of this correlation suggests that there are systematic effects of substituent pattern on the solvation energy of chlordecone congeners (Figure SI-2; Supporting Information).

Table 3. Calculated Gibbs Free Energy Values and Redox Potentials for the Reductive Dehalogenation of Chlordecone with $\mathrm{H}_{2}$ as Electron Donor ${ }^{a}$

\begin{tabular}{|c|c|c|c|c|}
\hline \multirow[t]{2}{*}{ substrate } & \multirow[t]{2}{*}{ product } & \multicolumn{2}{|c|}{$\Delta G^{0_{1}}$} & \multirow{2}{*}{$\begin{array}{l}E_{\mathrm{o}}{ }^{\prime} \\
\mathrm{mV}\end{array}$} \\
\hline & & $\mathrm{kJ} /$ reaction & $\mathrm{kJ} / \mathrm{Cl}$ removed & \\
\hline \multirow[t]{4}{*}{ chlordecone } & 10-hydrochlordecone & -158.4 & -158.4 & 407 \\
\hline & 9-hydrochlordecone & -159.3 & -159.3 & 411 \\
\hline & 8-hydrochlordecone & -159.7 & -159.7 & 413 \\
\hline & 6-hydrochlordecone & -155.5 & -155.5 & 392 \\
\hline chlordecone & decahydrochlordecone & -1448.1 & -144.8 & 336 \\
\hline
\end{tabular}

Reductive Dechlorination of Chlordecone. Table 3 shows that the reductive dechlorination of chlordecone with $\mathrm{H}_{2}$ as electron donor is exergonic with reaction free energies under standard conditions $(\mathrm{pH}=7)$ in the range of -145 to $-160 \mathrm{~kJ} / \mathrm{mol}$ of chlorine removed, depending on the dechlorination product formed. This implies that microorganisms can potentially obtain energy for growth from the reductive dechlorination of chlordecone: the $\Delta G^{o^{\prime}}$ for the dechlorination of chlordecone is well within the range of typical energy yields of -130 to $-180 \mathrm{~kJ} / \mathrm{mol}$ obtained from the dechlorination of a diverse series of chlorinated aliphatic and aromatic compounds that can support growth of the microorganisms involved. ${ }^{2,45}$ Per mol of reducing equivalents $\left(\mathrm{H}_{2}\right)$ consumed $\Delta G^{o^{\prime}}$ for the complete dechlorination of chlordecone to decahydrochlordecone is slightly less favorable than for its dechlorination to 
monohydrochlordecone, reflecting the fact that the chloro substituents in chlordecone consistently destabilize each other. At redox potentials $\left(E_{\circ}{ }^{\prime}\right)$ of 336 to $407 \mathrm{mV}$ (Table 3) the redox potential for chlordecone is similar to that of the redox couple $\mathrm{NO}_{3}{ }^{-} / \mathrm{NO}_{2}{ }^{-}\left(E_{\mathrm{o}}{ }^{\prime}=\right.$ $433 \mathrm{mV})$. This is substantially higher than the values for sulfate $\left(\mathrm{SO}_{4}{ }^{2-} / \mathrm{SH}^{-}\right)$and bicarbonate $\left(\mathrm{HCO}_{3}{ }^{-} / \mathrm{CH}_{4}\right)$. Based in these values reductive dechlorination of chlordecone is unlikely to occur under aerobic and FeIII reducing conditions. ${ }^{22,45}$

Energy of Dechlorination under in situ Conditions. Calculation of $\Delta G$ values for chlordecone dechlorination under in situ conditions requires information on the actual concentrations of substrates (chlordecone, $\mathrm{H}_{2}$ ) and products $\left(\mathrm{H}^{+}, \mathrm{Cl}^{-}\right.$and lesser chlorinated chlordecone congeners). We are not aware of any such data sets available in the literature, but lacking those we can make some assumptions. If we assume $\mathrm{pH}=7$, a chloride concentration of $1 \mathrm{mM}(35 \mathrm{mg} / \mathrm{L})$, a hydrogen concentration of $10 \mathrm{~Pa}$ (a value typical for methanogenic ecosystems) and concentrations of monohydrochlordecones that are 100 to 1000 fold lower than those of chlordecone (as they are in fish and shellfish) ${ }^{14}$ dechlorination of chlordecone under in situ conditions in methanogenic environments is 5.7 to $11.4 \mathrm{~kJ} / \mathrm{mol}$ more favorable than under standard conditions.

Potential Dechlorination Pathway. Based on the $\Delta G^{\mathrm{o}}$ values, dechlorination of chlordecone to a mixture of monohydrochlordecone congeners which are in thermodynamic equilibrium with chlordecone consists of 38\% 8-hydrochlordecone, $32 \%$ 9-hydrochlordecone, 23\% 10-hydrochlordecone and 7\% 6-hydrochlordecone. Thus a chlordecone dechlorination pathway governed by thermodynamic equilibria will consist of a complex mixture of a wide variety of congeners. A chlordecone dechlorination pathway governed by the rule that the dechlorination pathway solely follows the energetically most favorable steps ${ }^{46}$ will result in much simpler mixture of congeners, with only one congener per set of congeners with the same degree of chlorination. For the monohydro and dihydro congeners this would encompass 8-monohydrochlordecone (Table 3) that would later be transformed into 6,7-dihydrochlordecone (Table SI-3, Supporting Information). The differences between the Gibbs free energy of the reactions generating the different possible congeners may in some cases not be higher than the uncertainty in the estimated $\Delta_{\mathrm{f}} G_{\text {aq }}^{o}$ values (e.g. for the monohydrochlordecone the differences range from 0.4 to $4.2 \mathrm{~kJ} / \mathrm{mol}$ ). However, such small differences have been sufficient to explain for instance the formation or absence of formation of dechlorination products of hexachlorobenzene in the environment. ${ }^{47}$ Interestingly, 8-monohydrochlordecone is indeed the only monohydro congener that has been identified as chlordecone alteration product in soil and fish caught in the James River near Hopewell, although it cannot be excluded that this compound occurred as an impurity in the original chlordecone ${ }^{48,49}$ It is also the sole monohydrochlordecone to have been detected after photolysis $^{32,33}$ or aerobic microbial attack of chlordecone ${ }^{24}$. Nevertheless, beside the 8hydro the 10-monohydrochlordecone was also found upon dechlorination with vitamin $\mathrm{B}_{12 \mathrm{~S}}{ }^{25}$. The dihydrochlordecones that have been reported to be formed in some of these experiments are the $3,7^{24,33}$ and $c i s-8,10^{25}$ isomers that are only the third and sixth most favorable that can be formed from 8-monohydrochlordecone based on $\Delta G^{\circ}$ values (Table SI-3). These observations strongly suggest that in these cases dechlorination of chlordecone was subject to kinetic rather than thermodynamic limitations. 
Dechlorination versus Alcohol Formation. Chlordecol is the unique identified end product of chlordecone metabolism in man and some other mammals. ${ }^{50,51}$ Reduction of the keto group of chlordecone to the corresponding alcohol $\left(\Delta_{\mathrm{f}} G_{\text {aq }}^{o}\right.$ chlordecol $=162.3$ $\mathrm{kJ} / \mathrm{mol}$ ) with hydrogen as electron donor according to the equation chlordecone $+\mathrm{H}_{2} \rightarrow$ chlordecol has a $\Delta G^{o^{\prime}}$ of $-70.1 \mathrm{~kJ} /$ reaction. Thus reductive dechlorination of chlordecone is energetically more favorable than its reduction to chlordecol. In environments with a limited availability of reducing equivalents $\left(\mathrm{H}_{2}\right)$, competition between the two reactions for these equivalents should be in favor of the dechlorination. It is possible however that to maximize recovery of energy certain microorganisms may be able to perform both reactions sequentially or simultaneously. Both ketone and alcohol dechlorinated chlordecone were detected upon reaction with vitamin $\mathrm{B}_{12 \mathrm{~S}}{ }^{25}$ although the formation of the alcohols as analytical artifacts cannot be ruled out in these experiments. ${ }^{\mathbf{5 2}}$

Fermentation of Chlordecone. From the thermodynamic point of view the high chlorine content of the molecule makes chlordecone less stable: while mineralization of the completely dechlorinated congener according to $\mathrm{C}_{10} \mathrm{H}_{10} \mathrm{O}+19 \mathrm{H}_{2} \mathrm{O} \rightarrow 10 \mathrm{CO}_{2}+24$ $\mathrm{H}_{2}$ is endergonic under standard conditions $\left(\Delta G^{o^{\prime}}=390.9 \mathrm{~kJ} / \mathrm{mol}\right)$, mineralization of chlordecone according to the equation $\mathrm{C}_{10} \mathrm{Cl}_{10} \mathrm{O}+19 \mathrm{H}_{2} \mathrm{O} \rightarrow 10 \mathrm{CO}_{2}+10 \mathrm{H}^{+}+10 \mathrm{Cl}^{-}+$ $14 \mathrm{H}_{2}\left(\Delta G^{o^{\prime}}=-1056.9 \mathrm{~kJ} / \mathrm{mol}\right)$ is highly exergonic. This is because carbon-halogen bonds serve as electron acceptors for reducing equivalents generated in the mineralization of the carbon skeleton of the chlordecone molecule. It is tempting to speculate that this opens up the possibilities for organisms to ferment chlordecone into for example acetate and $\mathrm{HCl}$, according to $2 \mathrm{C}_{10} \mathrm{Cl}_{10} \mathrm{O}+24 \mathrm{H}_{2} \mathrm{O} \rightarrow 6 \mathrm{CO}_{2}+7 \mathrm{CH}_{3} \mathrm{COO}^{-}+27 \mathrm{H}^{+}+20 \mathrm{Cl}^{-}\left(\Delta G^{o^{\prime}}=\right.$ $-1389.1 \mathrm{~kJ} / \mathrm{mol}$ chlordecone). This could be organisms that ferment $\mathrm{C}_{10} \mathrm{H}_{10} \mathrm{O}$ according to $\mathrm{C}_{10} \mathrm{H}_{10} \mathrm{O}+7 \mathrm{H}_{2} \mathrm{O}+2 \mathrm{CO}_{2} \rightarrow 6 \mathrm{CH}_{3} \mathrm{COO}^{-}+6 \mathrm{H}^{+}\left(\Delta G^{o^{\prime}}=-178.6 \mathrm{~kJ}\right)$ with relaxed substrate specificity. Such organisms would obtain enough energy for growth from fermentation of the carbon backbone of the molecule.

Dichloroelimination. Dihaloelimination is a known dehalogenation pathway for removal of vicinal halo substituents in halogenated alkanes. ${ }^{53}$ Per mole of reducing equivalents used this pathway releases more energy than hydrogenolysis. ${ }^{22}$ However, dihaloelimination as a degradation mechanism for chlordecone is unlikely as it would lead to the formation of bridgehead double bonds, which is very improbable due to ring strain and angle strain in particular (violation of Bredt's rule). ${ }^{54,55}$

Chlordecone Mineralization. The Gibbs free energy of a mineralization reaction depends on the type of electron acceptor used, with $\mathrm{O}_{2}$ being the most favorable and $\mathrm{CO}_{2}$ the least favorable electron acceptor. Mineralization of chlordecone is exergonic with all the typical electron acceptors found in the environment (Table SI-4, Supporting Information).

Potential degradation pathway under aerobic conditions. With $\mathrm{O}_{2}$ as reactant the formation of the chlordecone lactone $\left(\Delta_{\mathrm{f}} G_{\text {aq }}^{o}=-239.6 \mathrm{~kJ} / \mathrm{mol}\right.$; Figure SI-3) according to the stoichiometry chlordecone $+1 / 2 \mathrm{O}_{2} \rightarrow$ chlordecone-lactone is thermodynamically 
favorable $\left(\Delta G^{o^{\prime}}=-147.6 \mathrm{~kJ}\right)$, suggesting that aerobic chlordecone degradation could proceed through a chlordecone lactone pathway. Lactone formation catalyzed by Flavindependent-Bayer-Villiger monooxygenases has been observed in other strained cage hydrocarbons such as adamantanone. ${ }^{56}$ However, the migrating ability of the alkyl group to form the lactone is decreased if an electron-withdrawing substituent is placed onto the alkyl group. ${ }^{57}$ In particular, a significant decrease in reaction rate was observed for $\alpha$ chloro ketone ${ }^{57}$ and as far as we know, Bayer-Villiger monooxygenases have failed to oxidize $\alpha$-chloro ketone (e.g. ref. 58). Given its structure chlordecone should therefore probably first be partially dehalogenated onto carbon atom 4 or/and 6 before chlordecone lactone formation becomes possible.

Fate of Chlordecone Degradation Products in the Environment. The volatility of chlordecone congeners, as characterized by the Henry coefficient, varies somewhat with the structure (Table SI-2, Supporting Information); there is no correlation between the number of chloro substituents and the volatility of the congener. This can be expected since volatility depends on a molecule's polarity as well as its mass. The solubility of chlordecone congeners as estimated with the EPA SPARC model ${ }^{59,60}$ increases with decreasing degree of chlorination (Figure SI-4, Supporting Information). Dechlorination products of chlordecone are thus likely to be more mobile in the environment than their parent compound. This could be a threat since less chlorinated congeners could be more toxic than the parent compound. For instance, vinylchloride, a degradation product of tetra- and trichloroethylene is more harmful than the parent compounds. ${ }^{61}$ However comparative toxicity test performed with the few chlordecone congeners available as pure compounds have shown that chlordecone alcohol toxicity $\geq$ chlordecone $>8$ monohydrochlordecone $>>>3,7$-dihydrochlordecone which suggests that the toxicity of chlordecone congeners decreases with decreasing degree of chlorination. ${ }^{50,62}$

Nevertheless, given the toxicity of the chlordecone itself there is an urgent need for methods that stimulate degradation of this compound in the environment. The present data indicate that there are no thermodynamic constraints which might preclude degradation of chlordecone in the environment and the existence of organisms that obtain energy for growth from the degradation and dechlorination of these compounds.

Steric Constraints and Kinetics. Reductive dechlorination involves the transfer of two electrons to a chlorinated hydrocarbon, and is often kinetically limited by the initial dissociative electron transfer to a $\mathrm{C}-\mathrm{Cl}$ bond. ${ }^{63}$ Whether reduction of a chlorinated hydrocarbon will take place is therefore best evaluated on the basis of one-electron reduction potentials. ${ }^{53}$ The present data set the framework for such an analysis: reductive dechlorination of chlordecone is thermodynamically favorable, but whether the reaction will actually proceed at environmentally significant rates and/or at rates which can sustain growth of organisms catalyzing such reactions remains to be determined. Steric factors will also play a role in this, especially in the issue of whether organisms can grow with chlordecone as electron acceptor. In this context it is important to note that (i) similar sized molecules such as PCBs and dioxins can serve as electron acceptors for organohalide respiring bacteria ${ }^{29}$ and (ii) compounds of the same substance class are generally dechlorinated faster if they contain a higher number of $\mathrm{Cl}$ atoms at the reactive C. ${ }^{53,64}$ The size and the highly oxidized carbon skeleton of the chlordecone molecule 
should thus be no impediment to the existence of chlordecone respiring bacteria. The xenobiotic nature of the molecule may well be a greater obstacle, but the observation that methanogens ${ }^{26}$ and reduced vitamin $\mathrm{B}_{12}$ can dechlorinate chlordecone ${ }^{25}$ implies that nature already has some of the essential building blocks to evolve such organisms.

Environmental Processes on the French West Indies. Chlordecone fate in the French West Indies can be explained by leaching solely, with no indication of endogenous microorganisms capable of chlordecone degradation. ${ }^{12}$ This suggests that bioremediation of chlordecone hotspots will require at least biostimulation, at best bioaugmentation. Reductive dechlorination functions best under reducing conditions, thus biostimulation should rationally encompass stimulation of anaerobic conditions for example by adding sugar cane waste to known chlordecone hotspots. Bioaugmentation would encompass adding chlordecone degraders. Such organisms may well be present in anaerobic niches on the islands, such as sediments and local anaerobic wastewater treatment systems. ${ }^{65}$ A viable alternative may also be the use of zero-valent iron or similar in situ chemical reduction technologies. ${ }^{66}$ All these remediation options will have to face the fact that most of the FWI polluted soils are andosols where chlordecone is strongly bound to the clay-organic matrix which makes it poorly available to chemical or biological agents. ${ }^{67}$ This, combined with organic matter stabilization in the fractal pore structure of andosols giving the soils an oxic character and resulting in a lack of reducing equivalents needed for reductive dechlorination possibly explains why natural attenuation of chlordecone has not been observed so far on the FWI. ${ }^{12,68}$

\section{AUTHOR INFORMATION}

\section{Corresponding Author}

*Phone +44 191222 8352; e-mail: jan.dolfing@ncl.ac.uk

\section{ACKNOWLEDGEMENTS}

This work benefited from the computing facilities at the "Centre Régional de Compétences en Modélisation Moléculaire de Marseille (CRCMM)" made available by Professor Didier Siri. Part of this work was supported by INRA (DEMICHLORD call for projects in the frame of the French Chlordecone National Action Plan) and by the European Union (FEDER Martinique 2007-2013). JD gratefully acknowledges travel support from Thierry Woignier (CNRS-IMBE). HM thanks Elisée Ferré (Aix-Marseille University - IMBE) for the loan of a molecular model that was very helpful to visualize the potential dechlorination products. All authors thank the fruitful inputs of the anonymous referees.

\section{ASSOCIATED CONTENT}

Supporting Information Available. Structures of all chlordecone potential dechlorinated congeners and their IUPAC and CAS names (Table SI-1); Calculated $\Delta_{\mathrm{f}} G^{\circ}$, $\Delta_{\mathrm{f}} H^{0}$, and $S^{\mathrm{o}}$ data for 62 chlordecone congeners plus corresponding Henry constants, strain energy (SE) and dipole ( $\mu$ ) values (Table SI-2); Calculated $\Delta G^{{ }^{\prime} \text { values for }}$ reductive dechlorination of monohydrochlordecone to dihydrochlordecone (Table SI-3); 
Change in Gibbs free energy $\left(\Delta G^{0}\right.$ in $\left.\mathrm{kJ} / \mathrm{mol}\right)$ for mineralization of chlordecone with various electron acceptors (Table SI-4); correlation between calculated $\Delta_{\mathrm{f}} G^{\mathrm{O}}$ gas and strain energy for various classes of chlordecone congeners (Figure SI-1); the correlation between calculated $\Delta G_{\mathrm{f}}^{\mathrm{o} \text { aq }}$ and $\Delta G_{\mathrm{f}}^{\mathrm{o} \text { gas }}$ for the 62 chlordecone congeners listed in Table SI-2 (Figure SI-2); the structures of 'chlordecone lactone' plus its calculated $\Delta_{\mathrm{f}} G^{0}$ and $\Delta_{\mathrm{f}} H^{0}$ values (Figure SI-3); Estimated solubility of chlordecone congeners versus their degree of chlorination (Figure SI-4). This information is available free of charge via the Internet at http://pubs.acs.org/

\section{REFERENCES}

(1) UNEP/POPS/POPRC.1/INF/6 United Nations Environment Programme, Stockholm Convention on Persistent Organic Pollutants. Persistent Organic Pollutants Review Committee, Consideration of chemicals proposed for inclusion in Annexes A, B and C of the Convention: Chlordecone. Geneva, Switzerland, 2005. Link access:

http://212.203.125.111/Convention/POPsReviewCommittee/POPRCMeetings/PO PRC1documents/tabid/108/language/en-US/Default.aspx

(2) UNEP/POPS/POPRC.1/10. United Nations Environment Programme, Stockholm Convention on Persistent Organic Pollutants. Persistent Organic Pollutants Review Committee, Decision POPRC-1/4: Chlordecone. Geneva, Switzerland, 2005

http://www.pops.int/documents/meetings/poprc/meeting docs/reports/report E.pd $\underline{\mathrm{f}}$

(3) UNEP/POPS/POPRC.3/20/Add.10. United Nations Environment Programme, Stockholm Convention on Persistent Organic Pollutants. Persistent Organic Pollutants Review Committee, Third meeting. Revised risk profile on chlordecone. Geneva, Switzerland, 2007. http://www.pops.int/documents/meetings/poprc/POPRC3/POPRC3_Report_e/PO PRC3 Report add10 e.pdf

(4) UNEP/POPS/POPRC.3/20/Add.2. United Nations Environment Programme, Stockholm Convention on Persistent Organic Pollutants. Persistent Organic Pollutants Review Committee, Third meeting. Risk management evaluation on chlordecone. Geneva, Switzerland, 2007. http://www.pops.int/documents/meetings/poprc/POPRC3/POPRC3 Report e/PO PRC3_Report_add2_e.pdf

(5) Dawson, G. W.; Weimer, W. C.; Shupe, S. J. Kepone - a case study of persistent material. The American Institute of Chemical Engineers (AIChE) Symposium Series 1979, 75 (190), 366-375.

(6) Huggett R. J. Kepone and the James River. In Contaminated Marine Sediments: Assessments and Remediation. National Academic Press: Washington, DC, 1989; pp 417-424.

(7) Huggett, R. J.; Bender, M. E. Kepone in the James River. Environ. Sci. Technol. 1980, 14 (8), 918-923.

(8) Le Déaut, J. Y.; Procaccia, C. Pesticide use in the Antilles: current situation and perspectives for change. OPECST (Office Parlementaire d'Evaluation des Choix 
Scientifiques et Technologique), 2009.

http://www.senat.fr/opecst/resume/4pagesbanane anglais.pdf. A full version is available (in French only) at http://www.senat.fr/rap/r08-487/r08-4871.pdf

(9) United Nations; Stockholm Convention on Persistent Organic Pollutants, Depository Notification C.N.524.2009.TREATIES-4, New York, 2009. http://chm.pops.int/Portals/0/download.aspx?d=UNEP-POPS-TREATY-NOTIFCN524-2009.En.pdf

(10) Lohmann, R.; Breivik, K.; Dach, J.; Muir, D. Global fate of POPs: Current and future research directions. Environ. Poll. 2007, 150 (1), 150-165

(11) Brunet, D.; Woignier, T.; Lesueur-Jannoyer, M.; Achard, R.; Rangon, L.; Barthès, B. G. Determination of soil content in chlordecone (organochlorine pesticide) using near infrared reflectance spectroscopy (NIRS). Environ. Poll. 2009, 157 (11), 3120-3125.

(12) Cabidoche, Y. -M.; Achard, R.; Cattan, P.; Clermont-Dauphin, C.; Massat, F.; Sansoulet, J. Long-term pollution by chlordecone of tropical volcanic soils in the French West Indies: A simple leaching model accounts for current residue. Environ. Poll. 2009, 157 (5), 1697-1705.

(13) Coat, S.; Bocquené, G.; Godard, R. Contamination of some aquatic species with the organochlorine pesticide chlordecone in Martinique. Aquat. Living Resources 2006, 19 (2), 181-187.

(14) Coat, S.; Monti, D.; Legendre, P.; Bouchon, C.; Massat, F.; Lepoint, G. Organochlorine pollution in tropical rivers (Guadeloupe): Role of ecological factors in food web bioaccumulation. Environ. Poll. 2011, 159 (6), 1692-1701.

(15) Gourcy, L.; Baran, N.; Vittecoq, B. Improving the knowledge of pesticide and nitrate transfer processes using age-dating tools $\left(\mathrm{CFC}, \mathrm{SF}_{6},{ }^{3} \mathrm{H}\right)$ in a volcanic island (Martinique, French West Indies). J. Contam. Hydrol. 2009, 108 (3-4), 107-117.

(16) Multigner, L.; Kadhel, P.; Huc-Terki, F.; Thome, J. P.; Janky, E.; Auger, J. Exposure to chlordecone and male fertility in Guadeloupe (French West Indies). Epidemiology 2006, 17 (6), S372.

(17) Multigner, L.; Cordier, S.; Kadhel, P.; Huc-Terki, F.; Blanchet, P.; Bataille, H.; Janky, E. 2007. Pollution par le chlordécone aux Antilles. Quel impact sur la santé de la population? Environnement, Risques et Santé 2007, 6, 405-407.

(18) Multigner, L.; Ndong, J.R.; Giusti, A.; Romana, M.; Delacroix-Maillard, H.; Cordier, S.; Jégou, B.; Thome, J. P.; Blanchet, P. Chlordecone exposure and risk of prostate cancer. J. Clin. Oncol. 2010, 28 (21), 3457-3462.

(19) Guldner, L.; Multigner, L.; Heraud, F.; Monfort, C.; Thome, J. P.; Giusti, A.; Kadhel, P.; Cordier, S. Pesticide exposure of pregnant women in Guadeloupe: Ability of a food frequency questionnaire to estimate blood concentration of chlordecone. Environ. Res. 2010, 110 (2), 146-151.

(20) Bossert, I. D.; Häggblom, M. M.; Young, L. Y. Microbial Ecology of Dehalogenation. In Dehalogenation: Microbial Processes and Environmental Applications, Häggblom M. M., Bossert, I. D., Eds.; Kluwer: Boston, MA, 2003; pp. 33-52.

(21) Smidt, H.; de Vos, W. M. Anaerobic dehalogenation. Annu. Rev. Microbiol. 2004, $58,43-73$. 
(22) Dolfing, J. Thermodynamic Considerations for Dehalogenation In Dehalogenation: Microbial Processes and Environmental Applications, Häggblom M. M., Bossert, I. D., Eds.; Kluwer: Boston, MA, 2003; pp. 89-114.

(23) Motl, M. L. EPA develops process to destroy kepone. Environ. Manag. 1977, 1 (6), 491-493.

(24) Orndorff, S. A.; Colwell, R. R. Microbial transformation of Kepone. Appl. Environ. Microbiol. 1980, 39 (2), 398-406.

(25) Schrauzer, G. N.; Katz, R. N. Reductive dechlorination and degradation of Mirex and Kepone with vitamin $\mathrm{B}_{12 \mathrm{~s}}$. Bioinorg. Chem. 1978, 9 (2), 123-143.

(26) Jablonski, P. E.; Pheasant, D. J.; Ferry, J. G. Conversion of Kepone by Methanosarcina thermophila. FEMS Microbiol. Lett. 1996, 139 (2-3), 169-173.

(27) Hedderich, R.; Whitman, W. B. Physiology and biochemistry of methaneproducing Archaea. Prokaryotes 2006, 2, 1050-1079.

(28) van Eekert, M. H. A.; Stams, A. J. M.; Field, J. A.; Schraa, G. Gratuitous dechlorination of chloroethanes by methanogenic granular sludge. Appl. Microbiol. Biotechnol. 1999, 51 (1), 46-52.

(29) Maphosa, F.; de Vos, W. M.; Smidt, H. Exploiting the ecogenomics toolbox for environmental diagnostics of organohalide-respiring bacteria. Trends Biotechnol. 2010, 28 (6), 308-316.

(30) Marchand, A. P. Synthesis and chemistry of homocubanes, bishomocubanes and trishomocubanes. Chem. Rev. 1989, 89 (5), 1011-1033.

(31) Dilling, W. L.; Bishomocubane chemistry. 14. Molecular mechanics calculations on bishomocubyl systems. J. Org. Chem. 1993, 58 (20), 5338-5344.

(32) Alley, E. A.; Layton, B. R.; Minyard, P. Identification of the photoproducts of the insecticides mirex and kepone. J. Agric. Food Chem. 1974, 22 (3), 442-445.

(33) Wilson, N. K.; Zehr, R. D. Structures of some kepone photoproducts and related chlorinated pentacyclodecanes by carbon-13 and proton nuclear magnetic resonance. J. Org. Chem. 1979, 44 (8), 1278-1282.

(34) Frisch, M. J.; Trucks, G. W.; Schlegel, H. B.; Scuseria, G. E.; Robb, M. A.; Cheeseman, J. R.; Zakrzewski, V. G.; Montgomery, J. A., Jr.; Stratmann, R. E.; Burant, J. C.; Dapprich, S.; Millam, J. M.; Daniels, A. D.; Kudin, K. N.; Strain, M. C.; Farkas, O.; Tomasi, J.; Barone, V.; Cossi, M.; Cammi, R.; Mennucci, B.; Pomelli, C.; Adamo, C.; Clifford, S.; Ochterski, J.; Petersson, G. A.; Ayala, P. Y.; Cui, Q.; Morokuma, K.; Malick, D. K.; Rabuck, A. D.; Raghavachari, K.;

Foresman, J. B.; Cioslowski, J.; Ortiz, J. V.; Stefanov, B. B.; Liu, G.; Liashenko, A.; Piskorz, P.; Komaromi, I.; Gomperts, R.; Martin, R. L.; Fox, D. J.; Keith, T.; Al-Laham, M. A.; Peng, C. Y.; Nanayakkara, A.; Gonzalez, C.; Challacombe, M.; Gill, P. M. W.; Johnson, B.; Chen, W.; Wong, M. W.; Andres, J. L.; Gonzalez, C.; Head-Gordon, M.; Replogle, E. S.; Pople, J. A., GAUSSIAN 03, Revision E1, Gaussian: Pittsburgh PA, 2003.

(35) Baboul, A. G.; Curtiss, L. A.; Redfern, P. C.; Raghavachari, K. Gaussian-3 theory using density functional geometries and zero-point energies. J. Chem. Phys. 1999, 110 (16), 7650-7657.

(36) Fabian, W. M. F. Accurate thermochemistry from quantum chemical calculations? Monatsh. Chem. 2008, 139 (4), 309-318. 
(37) Roux, M. V.; Davalos, J. Z.; Jimenez, P.; Notario, R.; Castano, O.; Chickos, J. S.; Hanshaw, W.; Zhao, H.; Rath, N.; Liebman, J. F.; Farivar, B. S.; BashirHashemi, A. Cubane, cuneane, and their carboxylates: A calorimetric, crystallographic, calculational, and conceptual coinvestigation. J. Org. Chem. 2005, 70 (14), 5461-5470.

(38) Tomasi, J.; Mennucci, B.; Cammi, R. Quantum mechanical solvation models. Chem. Rev. 2005, 105 (8), 2999-3093.

(39) Cox, J. D.; Wagman, D. D.; Medvedev, V. A. CODATA Key Values for Thermodynamics. Hemisphere Publishing, New York, 1989. http://www.codata.org/resources/databases/key1.html

(40) Thauer, R. K.; Jungermann, K.; Decker, K. Energy conservation in chemotrophic anaerobic bacteria. Bacteriol. Rev. 1977, 41 (1), 100-180.

(41) Aylward, G.; Findlay, T. SI Chemical Data, $6^{\text {th }}$ ed., Wiley 2008.

(42) Holmes, D. A.; Harrison, B. K.; Dolfing, J. Estimation of Gibbs free energies of formation for polychlorinated biphenyls. Environ. Sci. Technol. 1993, 27 (4), $725-$ 731.

(43) Huang, C.,-L.; Harrison, B. K.; Madura, J.; Dolfing, J. Gibbs free energies of formation of PCDDs: Evaluation of estimation methods and application for predicting dehalogenation pathways. Environ. Toxicol. Chem. 1996, 15 (6), 824836.

(44) Dolfing, J.; Janssen, D. B. Estimates of Gibbs free energies of formation of chlorinated aliphatic compounds. Biodegradation 1994, 5 (1), 21-28.

(45) Dolfing, J.; Harrison, B. K. Gibbs free energy of formation of halogenated aromatic compounds and their potential role as electron acceptors in anaerobic environments. Environ. Sci. Technol. 1992, 26 (11), 2213-2218.

(46) Dolfing, J.; Harrison, B. K. Redox and reduction potentials as parameters to predict the degradation pathway of chlorinated benzenes in anaerobic environments. FEMS Microbiol. Ecol. 1993, 13 (1), 23-30.

(47) Beurskens, J. E. M.; Dekker, C. G. C.; van den Heuvel, H.; Swart, M.; de Wolf, J.; Dolfing, J. Dechlorination of chlorinated benzenes by an anaerobic microbial consortium that selectively mediates the thermodynamic most favorable reactions. Environ. Sci. Technol. 1994, 28 (4), 701-706.

(48) Borsetti, A. P.; Roach, J. A. G. Identification of Kepone alteration products in soil and mullet. Bull. Environ. Contam. Toxicol. 1978, 20 (2), 241-247.

(49) Carver, R. A.; Griffith, F. D. Determination of Kepone dechlorination products in finfish, oysters and crustaceans. J. Agric. Food Chem. 1979, 27 (5), 1035-1037.

(50) Houston, T. E.; Mutter, L. C.; Blanke, V.; Guzelian, P. S. Chlordecone alcohol formation in the Mongolian gerbil (Meriones unguiculatus): A model for human metabolism of chlordecone (Kepone). Fund. Appl. Toxicol. 1981, 1 (3), 293-298.

(51) Molowa, D. T.; Shayne, A. G.; Guzelian, P. S. Purification and characterization of chlordecone reductase from human liver. J. Biol. Chem. 1986, 261 (27), 1262412627.

(52) Soine, W. H.; Forrest, T. T.; Smith, J. D.; Thermal reduction of chlordecone in the presence of alcohol. J. Chromatogr. 1983, 281, 95-99.

(53) Elsner, M.; Hofstetter, T. B. Current perspectives on the mechanisms of chlorohydrocarbon degradation in subsurface environments: Insights from 
kinetics, product formation, probe molecules, and isotope fractionation. In Aquatic Redox Chemistry; Tratnyek, P., et al., Eds.; ACS Symposium Series; American Chemical Society: Washington, DC, 2011; pp. 407-439.

(54) IUPAC. Compendium of Chemical Terminology, 2nd ed.; (the "Gold Book"). Compiled by A. D. McNaught and A. Wilkinson. Blackwell Scientific Publications: Oxford, 1997. doi:10.1351/goldbook.B00732

(55) Warner, P. M. Strained bridgehead double bonds. Chem. Rev. 1989, 89, 10671093.

(56) Selifonov, S. A. Microbial oxidation of adamantanone by Pseudomonas putida carrying the camphor catabolic plasmid. Biochem. Biophys. Res. Commun., 1992, $186(3), 1429-1436$.

(57) Grein, F.; Chen, C. A.; Edwards, D.; Crudden, C. M. Theoretical and experimental studies on the Baeyer-Villiger oxidation of ketones and the effect of $\alpha$-halo substituents. J. Org. Chem. 2006, 71 (3), 861-872.

(58) Fairlamb, I. J. S.; Grant, S.; Grogan, G.; Maddrell, D. A.; Nichols, J. C. A one-pot remote allylic hydroxylation and Baeyer-Villiger oxidation of a bicycle[3.2.0]hept-2-en-6-one by Cunninghamella echinulata NRRL 3655. Org. Biomol. Chem. 2004, 2 (13), 1831-1833.

(59) SPARC on line calculator, release V4.5, 2011. http://sparc.chem.uga.edu/sparc/

(60) Arp, H. P. H.; Droge, S. T. J.; Endo, S.; Giger, W.; Goss, K.-U.; Hawthorne, S. B.; Mabury, S. A.; Mayer, P.; McLachlan, M. S.; Pankow, J. F.; Schwarzenbach, R. P.; Wania, F.; Xing, B. More of EPA's SPARC online calculator-the need for high-quality predictions of chemical properties. Environ. Sci. Technol. 2010, 44 (12), 4400-4401

(61) McCarty, P. L. Breathing with chlorinated solvents. Science 1997, 276 (5318), 1521-1522.

(62) Soileau, S. D.; Moreland, D. F. Effects of chlordecone and its alteration products on isolated rat liver mitochondria. Toxicol. Appl. Pharmacol. 1983, 67, 89-99.

(63) Costentin, C.; Robert, M.; Saveant, J. M. Successive removal of chloride ions from organic polychloride pollutants. Mechanisms of reductive electrochemical elimination in aliphatic gem-polychlorides, $\alpha, \beta$-polychloroalkenes, and $\alpha, \beta$ polychloroalkanes in mildly protic medium. J. Am. Chem. Soc. 2003, 125 (35), 10729-10739.

(64) Vogel, T. M.; Criddle, C. S.; McCarty, P. L.; Transformations of halogenated aliphatic compounds. Environ. Sci. Technol. 1987, 21 (8), 722-736.

(65) Macarie, H.; Dolfing, J. La chlordécone (CLD) est-elle véritablement réfractaire à une dégradation microbienne? in Lesueur-Jannoyer, M. ed. Les Cahiers du PRAM. Le Lamentin, France: Pôle de Recherche Agro-Environmental de la Martinique, 2011, pp. 25-30.

(66) Dolfing, J.; van Eekert, M.; Seech, A.; Vogan, J.; Mueller, J. In situ chemical reduction (ISCR) technologies: Significance of low Eh reactions. Soil Sed. Contam. 2008, 17 (1), 63-74.

(67) Woignier, T.; Morell, M.; Morell, O.; Duffours, L.; Soler, A. Low water transport in fractal microstructure of tropical soils: application to chlordecone pesticide trapping. Ecohydrol. Hydrobiol. 2011, 11 (1-2), 121-128. 
(68) Chevallier, T.; Woignier, T.; Toucet, J.; Blanchart, E. Organic carbon stabilization in the fractal pore structure of andosols. Geoderma 2010, 159 (1-2), 182-188.

(69) Stumm, W.; Morgan, J.J. Aquatic Chemistry, $3^{\text {rd }}$ edition. John Wiley: New York, NY, 1996. 


\title{
SUPPORTING INFORM ATION
}

\section{GibbsFree Energy of Formation of Chlordecone and Potential Degradation Products: Implications for Remediation Strategiesand Environmental Fate}

\author{
Jan Dolfing ${ }^{\dagger *}$, Igor Novak ${ }^{\ddagger}$, Alain Archelas ${ }^{\S}$ and Hervé Macarie
}

\begin{abstract}
${ }^{\dagger}$ School of Civil Engineering \& Geosciences, Newcastle University, Newcastle-upon-Tyne, NE1 7RU, United Kingdom

${ }^{\sharp}$ Charles Sturt University, Orange, NSW 2800, Australia

$\S$ Aix-Marseille Université, CNRS, UMR ISM2, case 332, Faculté des Sciences et Techniques de St Jérome, Avenue Escadrille Normandie Niemen, F-13397 Marseille Cedex 20, France "IRD, UMR IMBE, Pôle de Recherche Agro-environnemental de la Martinique, Quartier Petit Morne, BP 214, F-97285 Lamentin, France
\end{abstract}

*Corresponding author: Jan.Dolfing@ncl.ac.uk

\section{CONTENTS:}

Table SI-1. Structures of all chlordecone potential dechlorinated congeners and their IUPAC and CAS names.

Table SI-2. Calculated $\Delta_{\mathrm{f}} G^{\circ}, \Delta_{\mathrm{f}} H^{\circ}$, and $S$ data for 62 chlordecone congeners plus corresponding Henry constants, strain energy (SE) and dipole $(\mu)$ values.

Table SI-3. Calculated $\Delta G^{\prime \prime}$ values for reductive dechlorination of monohydrochlordecone to dihydrochlordecone.

Table SI-4. Change in Gibbs free energy $\left(\Delta G^{\prime \prime}\right.$ in $\mathrm{kJ} / \mathrm{mol}$ ) for mineralization of chlordecone with various electron acceptors.

Figure SI-1. Correlation between calculated $\Delta_{\mathrm{f}} G_{\text {gas }}^{0}$ and strain energy for various classes of chlordecone congeners.

Figure SI-2. The correlation between calculated $\Delta_{\mathrm{f}} G_{\text {aq }}^{0}$ and $\Delta_{\mathrm{f}} G_{\text {gas }}^{0}$ for the 62 chlordecone congeners listed in Table SI-2.

Figure SI-3. The structure of 'chlordecone lactone' plus its calculated $\Delta_{\mathrm{f}} G^{\mathrm{o}}$ and $\Delta_{\mathrm{f}} H^{\mathrm{p}}$ values. Figure SI-4. Estimated solubility of chlordecone congeners versus their degree of chlorination. 
Gibbs Free Energy of Formation of Chlordecone and Potential Degradation Products: Implications for Remediation Strategies and Environmental Fate Table SI-1 Structures of all chlordecone potential dechlorinated congeners and their IUPAC and CAS names

\begin{tabular}{|c|c|c|c|c|c|}
\hline Entry & $\overline{\mathrm{nCl}}$ & Structure $^{\mathrm{a}}$ & IUPAC - Name & CAS - Name & Chirality \\
\hline 1 & 0 & & decachloropentacyclo[5.3.0.0 $\left.0^{2,6} \cdot 0^{3,9} \cdot 0^{4,8}\right]$ decan-5-one & $\begin{array}{l}\text { 1,1a,3,3a,4,5,5,5a,5b,6- } \\
\text { decachlorooctahydro-1,3,4- } \\
\text { metheno-2H-cyclobuta[cd]pentalen- } \\
\text { 2-one }\end{array}$ & meso \\
\hline 2 & 1 & & $\begin{array}{l}1,2,3,4,6,7,8,9,10- \\
\left.\text { nonachloropentacyclo[5.3.0.0 } 0^{2,6} \cdot 0^{3,9} \cdot 0^{4,8}\right] \text { decan-5-one }\end{array}$ & $\begin{array}{l}\text { 1,1a,3,3a,4,5,5a,5b,6- } \\
\text { nonachlorooctahydro-1,3,4- } \\
\text { metheno-2H-cyclobuta[cd]pentalen- } \\
\text { 2-one }\end{array}$ & meso \\
\hline 3 & 1 & $\mathbf{H}$ & $\begin{array}{l}1,2,3,4,6,7,8,10,10- \\
\left.\text { nonachloropentacyclo[5.3.0.0 } 0^{2,6} \cdot 0^{3,9} \cdot 0^{4,8}\right] \text { decan-5-one }\end{array}$ & $\begin{array}{l}\text { 1,1a,3,3a,4,5,5,5b,6- } \\
\text { nonachlorooctahydro-1,3,4- } \\
\text { metheno-2H-cyclobuta[cd]pentalen- } \\
\text { 2-one }\end{array}$ & meso \\
\hline 4 & 1 & & $\begin{array}{l}1,2,3,4,7,8,9,10,10- \\
\left.\text { nonachloropentacyclo[5.3.0.0 } 0^{2,6} \cdot 0^{3,9} \cdot 0^{4,8}\right] \text { decan-5-one }\end{array}$ & $\begin{array}{l}\text { 1,1a,3a,4,5,5,5a,5b,6- } \\
\text { nonachlorooctahydro-1,3,4- } \\
\text { metheno-2H-cyclobuta[cd]pentalen- } \\
\text { 2-one }\end{array}$ & meso \\
\hline 5 & 1 & & $\begin{array}{l}1,2,3,4,6,7,9,10,10- \\
\left.\text { nonachloropentacyclo[5.3.0.0 } 0^{2,6} \cdot 0^{3,9} \cdot 0^{4,8}\right] \text { decan-5-one }\end{array}$ & $\begin{array}{l}\text { 1,1a,3,3a,4,5,5,5a,5b- } \\
\text { nonachlorooctahydro-1,3,4- } \\
\text { metheno-2H-cyclobuta[cd]pentalen- } \\
\text { 2-one }{ }^{c}\end{array}$ & chiral \\
\hline 6 & 2 & & $\begin{array}{l}1,2,3,4,6,7,8,9- \\
\left.\text { octachloropentacyclo[5.3.0.0 } 0^{2,6} \cdot 0^{3,9} \cdot 0^{4,8}\right] \text { decan-5-one }\end{array}$ & $\begin{array}{l}\text { 1,1a,3,3a,4,5a,5b,6- } \\
\text { octachlorooctahydro-1,3,4-metheno- } \\
\text { 2H-cyclobuta[cd]pentalen-2-one }\end{array}$ & meso \\
\hline 7 & 2 & & $\begin{array}{l}1,2,3,6,7,8,10,10- \\
\left.\text { octachloropentacyclo[5.3.0.0 } 0^{2,6} \cdot 0^{3,9} \cdot 0^{4,8}\right] \text { decan-5-one }\end{array}$ & $\begin{array}{l}\text { 1,1a,3a,5,5,5a,5b,6- } \\
\text { octachlorooctahydro-1,3,4-metheno- } \\
\text { 2H-cyclobuta[cd]pentalen-2-one }\end{array}$ & meso \\
\hline
\end{tabular}


Gibbs Free Energy of Formation of Chlordecone and Potential Degradation Products: Implications for Remediation Strategies and Environmental Fate Table SI-1 Structures of all chlordecone potential dechlorinated congeners and their IUPAC and CAS names

\begin{tabular}{|c|c|c|c|c|c|}
\hline Entry & $\overline{\mathrm{nCl}}$ & Structure $^{a}$ & IUPAC - Name & CAS - Name & Chirality \\
\hline 8 & 2 & & $\begin{array}{l}1,3,4,6,8,9,10,10- \\
\left.\text { octachloropentacyclo[5.3.0.0 } 0^{2,6} \cdot 0^{3,9} \cdot 0^{4,8}\right] \text { decan-5-one }\end{array}$ & $\begin{array}{l}\text { 1,1a,3,4,5,5,5a,5b,- } \\
\text { octachlorooctahydro-1,3,4-metheno- } \\
\text { 2H-cyclobuta[cd]pentalen-2-one }\end{array}$ & meso \\
\hline 9 & 2 & & $\begin{array}{l}2,3,4,6,7,8,10,10- \\
\left.\text { octachloropentacyclo[5.3.0.0.6 } 0^{2,6} \cdot 0^{3,9} \cdot 0^{4,8}\right] \text { decan-5-one }\end{array}$ & $\begin{array}{l}\text { 1,1a,3,3a,5,5,5b,6- } \\
\text { octachlorooctahydro-1,3,4-metheno- } \\
\text { 2H-cyclobuta[cd]pentalen-2-one }\end{array}$ & meso \\
\hline 10 & 2 & & $\begin{array}{l}1,2,3,4,7,8,10,10- \\
\left.\text { octachloropentacyclo[5.3.0.0 } 0^{2,6} \cdot 0^{3,9} \cdot 0^{4,8}\right] \text { decan-5-one }\end{array}$ & $\begin{array}{l}\text { 1,1a,3a,4,5,5,5b,6- } \\
\text { octachlorooctahydro-1,3,4-metheno- } \\
\text { 2H-cyclobuta[cd]pentalen-2-one }\end{array}$ & meso \\
\hline 11 & 2 & & $\begin{array}{l}1,2,3,4,6,9,10,10- \\
\left.\text { octachloropentacyclo[5.3.0.0 } 0^{2,6} \cdot 0^{3,9} \cdot 0^{4,8}\right] \text { decan-5-one }\end{array}$ & $\begin{array}{l}\text { 1,1a,3,4,5,5,5a,6- } \\
\text { octachlorooctahydro-1,3,4-metheno- } \\
\text { 2H-cyclobuta[cd]pentalen-2-one }\end{array}$ & meso \\
\hline 12 & 2 & & $\begin{array}{l}1,2,3,7,8,9,10,10- \\
\left.\text { octachloropentacyclo[5.3.0.0 } 0^{2,6} \cdot 0^{3,9} \cdot 0^{4,8}\right] \text { decan-5-one }\end{array}$ & $\begin{array}{l}\text { 1,3a,4,5,5,5a,5b,6- } \\
\text { octachlorooctahydro-1,3,4-metheno- } \\
2 \mathrm{H} \text {-cyclobuta[cd]pentalen-2-one }\end{array}$ & meso \\
\hline 13 & 2 & & $\begin{array}{l}1,2,3,4,6,7,8,10- \\
\left.\text { octachloropentacyclo[5.3.0.0 } 0^{2,6} \cdot 0^{3,9} \cdot 0^{4,8}\right] \text { decan-5-one }\end{array}$ & $\begin{array}{l}\text { 1,1a,3,3a,4,5,5b,6- } \\
\text { octachlorooctahydro-1,3,4-metheno- } \\
2 \mathrm{H} \text {-cyclobuta[cd]pentalen-2-one }\end{array}$ & chiral \\
\hline 14 & 2 & & $\begin{array}{l}1, r-2,3,4,6,7,9, c-10- \\
\left.\text { octachloropentacyclo[5.3.0.0 } 0^{2,6} \cdot 0^{3,9} \cdot 0^{4,8}\right] \text { decan-5-one }\end{array}$ & $\begin{array}{l}r-1,1 \mathrm{a}, 3,3 \mathrm{a}, 4, c-5,5 \mathrm{a}, 5 \mathrm{~b}- \\
\text { octachlorooctahydro-1,3,4-metheno- } \text { 2H-cyclobuta[cd]pentalen-2-one }{ }^{\mathrm{H}}\end{array}$ & chiral \\
\hline
\end{tabular}


Gibbs Free Energy of Formation of Chlordecone and Potential Degradation Products: Implications for Remediation Strategies and Environmental Fate Table SI-1 Structures of all chlordecone potential dechlorinated congeners and their IUPAC and CAS names

\begin{tabular}{|c|c|c|c|c|c|}
\hline Entry & $\overline{\mathrm{nCl}}$ & Structure $^{\mathrm{a}}$ & IUPAC - Name & CAS - Name & Chirality \\
\hline 15 & 2 & $\mathbf{H}_{1}$ & $\begin{array}{l}1, r-2,3,4,6,7,9, t-10- \\
\left.\text { octachloropentacyclo[5.3.0.0 } 0^{2,6} \cdot 0^{3,9} \cdot 0^{4,8}\right] \text { decan-5-one }\end{array}$ & $\begin{array}{l}r-1,1 \mathrm{a}, 3,3 \mathrm{a}, 4, c-5,5 \mathrm{a}, 5 \mathrm{~b}- \\
\text { octachlorooctahydro-1,3,4-metheno- } \\
2 \mathrm{H}-\text {-yclobuta[cd]pentalen-2-one }^{c}\end{array}$ & chiral \\
\hline 16 & 2 & & $\begin{array}{l}1,2,3,4,7,8,9,10- \\
\left.\text { octachloropentacyclo[5.3.0.0 } 0^{2,6} \cdot 0^{3,9} \cdot 0^{4,8}\right] \text { decan-5-one }\end{array}$ & $\begin{array}{l}\text { 1,1a,3a,4,5,5a,5b,6- } \\
\text { octachlorooctahydro-1,3,4-metheno- } \\
\text { 2H-cyclobuta[cd]pentalen-2-one }\end{array}$ & chiral \\
\hline 17 & 2 & H & $\begin{array}{l}1,2,3,4,6,7,10,10- \\
\left.\text { octachloropentacyclo[5.3.0.0 } 0^{2,6} \cdot 0^{3,9} \cdot 0^{4,8}\right] \text { decan-5-one }\end{array}$ & $\begin{array}{l}\text { 1,1a,3,3a,5,5,5a,5b- } \\
\text { octachlorooctahydro-1,3,4-metheno- } \\
\text { 2H-cyclobuta[cd]pentalen-2-one }\end{array}$ & chiral \\
\hline 18 & 2 & & $\begin{array}{l}1,2,3,4,8,9,10,10- \\
\text { octachloropentacyclo }\left[5 \cdot 3 \cdot 0 \cdot 0^{2,6} \cdot 0^{3,9} \cdot 0^{4,8}\right] \text { decan-5-one }\end{array}$ & $\begin{array}{l}\text { 1,1a,3a,4,5,5,5a,5b- } \\
\text { octachlorooctahydro-1,3,4-metheno- } \\
\text { 2H-cyclobuta[cd]pentalen-2-one }\end{array}$ & chiral \\
\hline 19 & 2 & & $\begin{array}{l}1,2,3,4,6,8,10,10- \\
\left.\text { octachloropentacyclo[5.3.0.0 } 0^{2,6} \cdot 0^{3,9} \cdot 0^{4,8}\right] \text { decan-5-one }\end{array}$ & $\begin{array}{l}\text { 1,1a,3,3a,4,5,5,5b- } \\
\text { octachlorooctahydro-1,3,4-metheno- } \\
\text { 2H-cyclobuta[cd]pentalen-2-one }\end{array}$ & chiral \\
\hline 20 & 2 & & $\begin{array}{l}1,2,3,4,7,9,10,10- \\
\text { octachloropentacyclo }\left[5 \cdot 3 \cdot 0 \cdot 0^{2,6} \cdot 0^{3,9} \cdot 0^{4,8}\right] \text { decan-5-one }\end{array}$ & $\begin{array}{l}\text { 1,1a,3a,4,5,5,5a,6- } \\
\text { octachlorooctahydro-1,3,4-metheno- } \\
\text { 2H-cyclobuta[cd]pentalen-2-one }\end{array}$ & chiral \\
\hline 21 & 2 & & $\begin{array}{l}1,2,4,6,8,9,10,10- \\
\left.\text { octachloropentacyclo[5.3.0.0 } 0^{2,6} \cdot 0^{3,9} \cdot 0^{4,8}\right] \text { decan-5-one }\end{array}$ & $\begin{array}{l}\text { 1,1a,3,3a,4,5,5,5a- } \\
\text { octachlorooctahydro-1,3,4-metheno- } \\
\text { 2H-cyclobuta[cd]pentalen-2-one }\end{array}$ & chiral \\
\hline
\end{tabular}


Gibbs Free Energy of Formation of Chlordecone and Potential Degradation Products: Implications for Remediation Strategies and Environmental Fate Table SI-1 Structures of all chlordecone potential dechlorinated congeners and their IUPAC and CAS names

\begin{tabular}{|c|c|c|c|c|c|}
\hline Entry & $\overline{\mathrm{nCl}}$ & Structure $^{a}$ & IUPAC - Name & CAS - Name & Chirality \\
\hline 22 & 3 & & $\begin{array}{l}1,2,3,4,6,7,8- \\
\left.\text { heptachloropentacyclo[5.3.0.0 } 0^{2,6} \cdot 0^{3,9} \cdot 0^{4,8}\right] \text { decan-5-one }\end{array}$ & $\begin{array}{l}\text { 1,1a,3,3a,4,5b,6- } \\
\text { heptachlorooctahydro-1,3,4- } \\
\text { metheno-2H-cyclobuta[cd]pentalen- } \\
\text { 2-one }\end{array}$ & meso \\
\hline 23 & 3 & H & $\begin{array}{l}1,2,3,4,7,8,9- \\
\left.\text { heptachloropentacyclo[5.3.0.0 } 0^{2,6} \cdot 0^{3,9} \cdot 0^{4,8}\right] \text { decan-5-one }\end{array}$ & $\begin{array}{l}\text { 1,1a,3a,4,5a,5b,6- } \\
\text { heptachlorooctahydro-1,3,4- } \\
\text { metheno-2H-cyclobuta[cd]pentalen- } \\
\text { 2-one }\end{array}$ & meso \\
\hline 24 & 3 & & $\begin{array}{l}2,3,4,6,7,8,10- \\
\left.\text { heptachloropentacyclo[5.3.0.0 } 0^{2,6} \cdot 0^{3,9} \cdot 0^{4,8}\right] \text { decan-5-one }\end{array}$ & $\begin{array}{l}\text { 1,1a,3,3a,5,5b,6- } \\
\text { heptachlorooctahydro-1,3,4- } \\
\text { metheno-2H-cyclobuta[cd]pentalen- } \\
\text { 2-one }\end{array}$ & meso \\
\hline 25 & 3 & & $\begin{array}{l}1, r-2,3,4,6,9, c-10- \\
\left.\text { heptachloropentacyclo[5.3.0.0 } 0^{2,6} \cdot 0^{3,9} \cdot 0^{4,8}\right] \text { decan-5-one }\end{array}$ & $\begin{array}{l}r-1,1 \mathrm{a}, 3,4, c-5,5 \mathrm{a}, 6- \\
\text { heptachlorooctahydro-1,3,4- } \\
\text { metheno-2H-cyclobuta[cd]pentalen- } \\
\text { 2-one }\end{array}$ & meso \\
\hline 26 & 3 & & $\begin{array}{l}1,2,3,7,8,9,10- \\
\left.\text { heptachloropentacyclo[5.3.0.0 } 0^{2,6} \cdot 0^{3,9} \cdot 0^{4,8}\right] \text { decan-5-one }\end{array}$ & $\begin{array}{l}r-1,3 a, 4, t-5,5 a, 5 b, 6- \\
\text { heptachlorooctahydro-1,3,4- } \\
\text { metheno-2H-cyclobuta[cd]pentalen- } \\
\text { 2-one }\end{array}$ & meso \\
\hline 27 & 3 & & $\begin{array}{l}1,2,3,7,8,10,10- \\
\left.\text { heptachloropentacyclo[5.3.0.0 } 0^{2,6} \cdot 0^{3,9} \cdot 0^{4,8}\right] \text { decan-5-one }\end{array}$ & $\begin{array}{l}\text { 1,3a,4,5,5,5b,6- } \\
\text { heptachlorooctahydro-1,3,4- } \\
\text { metheno-2H-cyclobuta[cd]pentalen- } \\
\text { 2-one }\end{array}$ & meso \\
\hline 28 & 3 & & $\begin{array}{l}1, r-2,3,4,6,9, t-10- \\
\left.\text { heptachloropentacyclo[5.3.0.0 } 0^{2,6} \cdot 0^{3,9} \cdot 0^{4,8}\right] \text { decan-5-one }\end{array}$ & $\begin{array}{l}r-1,1 \mathrm{a}, 3,4, t-5,5 a, 6- \\
\text { heptachlorooctahydro-1,3,4- } \\
\text { metheno-2H-cyclobuta[cd]pentalen- } \\
\text { 2-one }\end{array}$ & meso \\
\hline
\end{tabular}


Gibbs Free Energy of Formation of Chlordecone and Potential Degradation Products: Implications for Remediation Strategies and Environmental Fate Table SI-1 Structures of all chlordecone potential dechlorinated congeners and their IUPAC and CAS names

\begin{tabular}{|c|c|c|c|c|c|}
\hline Entry & $\overline{\mathrm{nCl}}$ & Structure $^{a}$ & IUPAC - Name & CAS - Name & Chirality \\
\hline 29 & 3 & & $\begin{array}{l}2,3,4,7,8,10,10- \\
\left.\text { heptachloropentacyclo[5.3.0.0 } 0^{2,6} \cdot 0^{3,9} \cdot 0^{4,8}\right] \text { decan-5-one }\end{array}$ & $\begin{array}{l}\text { 1,1a,3a,5,5,5b,6- } \\
\text { heptachlorooctahydro-1,3,4- } \\
\text { metheno-2H-cyclobuta[cd]pentalen- } \\
\text { 2-one }\end{array}$ & meso \\
\hline 30 & 3 & H & $\begin{array}{l}1,3,4,6,8,10,10- \\
\left.\text { heptachloropentacyclo[5.3.0.0 } 0^{2,6} \cdot 0^{3,9} \cdot 0^{4,8}\right] \text { decan-5-one }\end{array}$ & $\begin{array}{l}1,1 \mathrm{a}, 3,4,5,5,5 \mathrm{~b}- \\
\text { heptachlorooctahydro-1,3,4- } \\
\text { metheno-2H-cyclobuta[cd]pentalen- } \\
\text { 2-one }\end{array}$ & meso \\
\hline 31 & 3 & & $\begin{array}{l}1,2,4,7,9,10,10- \\
\left.\text { heptachloropentacyclo[5.3.0.0 } 0^{2,6} \cdot 0^{3,9} \cdot 0^{4,8}\right] \text { decan-5-one }\end{array}$ & $\begin{array}{l}1,3,4,5,5,5 \mathrm{a}, 5 \mathrm{~b}- \\
\text { heptachlorooctahydro-1,3,4- } \\
\text { metheno-2H-cyclobuta[cd]pentalen- } \\
\text { 2-one } \text { b }^{\text {b }}\end{array}$ & meso \\
\hline 32 & 3 & & $\begin{array}{l}1,2,4,6,7,10,10- \\
\left.\text { heptachloropentacyclo[5.3.0.0 } 0^{2,6} \cdot 0^{3,9} \cdot 0^{4,8}\right] \text { decan-5-one }\end{array}$ & $\begin{array}{l}1,1 \mathrm{a}, 3,5,5,5 \mathrm{a}, 5 \mathrm{~b}- \\
\text { heptachlorooctahydro-1,3,4- } \\
\text { metheno-2H-cyclobuta[cd]pentalen- } \\
\text { 2-one } \text { b }^{\text {b }}\end{array}$ & meso \\
\hline 33 & 3 & & $\begin{array}{l}1,2,6,7,9,10,10- \\
\left.\text { heptachloropentacyclo[5.3.0.0 } 0^{2,6} \cdot 0^{3,9} \cdot 0^{4,8}\right] \text { decan-5-one }\end{array}$ & $\begin{array}{l}\text { 1,1a,4,5,5,5a,5b- } \\
\text { heptachlorooctahydro-1,3,4- } \\
\text { metheno-2H-cyclobuta[cd]pentalen- } \\
\text { 2-one } \text { b }^{\text {b }}\end{array}$ & méso \\
\hline 34 & 3 & & $\begin{array}{l}1,2,3,4,6,7,9- \\
\left.\text { heptachloropentacyclo[5.3.0.0 } 0^{2,6} \cdot 0^{3,9} \cdot 0^{4,8}\right] \text { decan-5-one }\end{array}$ & $\begin{array}{l}\text { 1,1a,3,3a,4,5a,6- } \\
\text { heptachlorooctahydro-1,3,4- } \\
\text { metheno-2H-cyclobuta[cd]pentalen- } \\
\text { 2-one }\end{array}$ & chiral \\
\hline 35 & 3 & & $\begin{array}{l}1, r-2,3,4,6,8, c-10- \\
\left.\text { heptachloropentacyclo[5.3.0.0 } 0^{2,6} \cdot 0^{3,9} \cdot 0^{4,8}\right] \text { decan-5-one }\end{array}$ & $\begin{array}{l}r-1,1 \mathrm{a}, 3,4, c-5,5 b, 6- \\
\text { heptachlorooctahydro-1,3,4- } \\
\text { metheno-2H-cyclobuta[cd]pentalen- } \\
\text { 2-one }{ }^{\text {b }}\end{array}$ & chiral \\
\hline
\end{tabular}


Gibbs Free Energy of Formation of Chlordecone and Potential Degradation Products: Implications for Remediation Strategies and Environmental Fate Table SI-1 Structures of all chlordecone potential dechlorinated congeners and their IUPAC and CAS names

\begin{tabular}{|c|c|c|c|c|c|}
\hline Entry & $\overline{\mathrm{nCl}}$ & Structure $^{a}$ & IUPAC - Name & CAS - Name & Chirality \\
\hline 36 & 3 & $\mathbf{H}$ & $\begin{array}{l}1, r-2,3,4,6,8, t-10- \\
\left.\text { heptachloropentacyclo[5.3.0.0 } 0^{2,6} \cdot 0^{3,9} \cdot 0^{4,8}\right] \text { decan-5-one }\end{array}$ & $\begin{array}{l}r-1,1 \mathrm{a}, 3,4, t-5,5 \mathrm{~b}, 6- \\
\text { heptachlorooctahydro-1,3,4- } \\
\text { metheno-2H-cyclobuta[cd]pentalen- } \\
\text { 2-one }{ }^{\mathrm{b}}\end{array}$ & chiral \\
\hline 37 & 3 & & $\begin{array}{l}1,2,3,4,7,8,10- \\
\left.\text { heptachloropentacyclo[5.3.0.0 } 0^{2,6} \cdot 0^{3,9} \cdot 0^{4,8}\right] \text { decan-5-one }\end{array}$ & $\begin{array}{l}\text { 1,1a,3a,4,5,5b,6- } \\
\text { heptachlorooctahydro-1,3,4- } \\
\text { metheno-2H-cyclobuta[cd]pentalen- } \\
\text { 2-one }\end{array}$ & chiral \\
\hline 38 & 3 & & $\begin{array}{l}1, r-2,3,4,7,9, c-10- \\
\left.\text { heptachloropentacyclo[5.3.0.0 } 0^{2,6} \cdot 0^{3,9} \cdot 0^{4,8}\right] \text { decan-5-one }\end{array}$ & $\begin{array}{l}r \text {-1,1a,3a,4,c-5,5a,6- } \\
\text { heptachlorooctahydro-1,3,4- } \\
\text { metheno-2H-cyclobuta[cd]pentalen- } \\
\text { 2-one }\end{array}$ & chiral \\
\hline 39 & 3 & $\mathbf{H}$ & $\begin{array}{l}1, r-2,3,4,7,9, t-10- \\
\left.\text { heptachloropentacyclo[5.3.0.0 } 0^{2,6} \cdot 0^{3,9} \cdot 0^{4,8}\right] \text { decan-5-one }\end{array}$ & $\begin{array}{l}r-1,1 \mathrm{a}, 3 \mathrm{a}, 4, t-5,5 \mathrm{a}, 6- \\
\text { heptachlorooctahydro-1,3,4- } \\
\text { metheno-2H-cyclobuta[cd]pentalen- } \\
\text { 2-one }\end{array}$ & chiral \\
\hline 40 & 3 & & $\begin{array}{l}1,2,4,6,8,9,10- \\
\left.\text { heptachloropentacyclo[5.3.0.0 } 0^{2,6} \cdot 0^{3,9} \cdot 0^{4,8}\right] \text { decan-5-one }\end{array}$ & $\begin{array}{l}\text { 1,1a,3,3a,4,5,5a- } \\
\text { heptachlorooctahydro-1,3,4- } \\
\text { metheno-2H-cyclobuta[cd]pentalen- } \\
\text { 2-one }{ }^{\text {b }}\end{array}$ & chiral \\
\hline 41 & 3 & & $\begin{array}{l}1, r-2,3,4,6,7, c-10- \\
\left.\text { heptachloropentacyclo[5.3.0.0 } 0^{2,6} \cdot 0^{3,9} \cdot 0^{4,8}\right] \text { decan-5-one }\end{array}$ & $\begin{array}{l}r-1,1 \mathrm{a}, 3,3 \mathrm{a}, 4, c-5,6- \\
\text { heptachlorooctahydro-1,3,4- } \\
\text { metheno-2H-cyclobuta[cd]pentalen- } \\
\text { 2-one }\end{array}$ & chiral \\
\hline
\end{tabular}


Gibbs Free Energy of Formation of Chlordecone and Potential Degradation Products: Implications for Remediation Strategies and Environmental Fate Table SI-1 Structures of all chlordecone potential dechlorinated congeners and their IUPAC and CAS names

\begin{tabular}{|c|c|c|c|c|c|}
\hline Entry & $\overline{\mathrm{nCl}}$ & Structure $^{\mathrm{a}}$ & IUPAC - Name & CAS - Name & Chirality \\
\hline 42 & 3 & & $\begin{array}{l}1, r-2,3,4,6,7, t-10- \\
\left.\text { heptachloropentacyclo[5.3.0.0 } 0^{2,6} \cdot 0^{3,9} \cdot 0^{4,8}\right] \text { decan-5-one }\end{array}$ & $\begin{array}{l}r-1,1 \mathrm{a}, 3,3 \mathrm{a}, 4, t-5,6- \\
\text { heptachlorooctahydro-1,3,4- } \\
\text { metheno-2H-cyclobuta[cd]pentalen- } \\
\text { 2-one }\end{array}$ & chiral \\
\hline 43 & 3 & $\mathbf{H}$ & $\begin{array}{l}1,2,3,6,7,8,10- \\
\left.\text { heptachloropentacyclo[5.3.0.0 } 0^{2,6} \cdot 0^{3,9} \cdot 0^{4,8}\right] \text { decan-5-one }\end{array}$ & $\begin{array}{l}\text { 1,1a,3a,5,5a,5b,6- } \\
\text { heptachlorooctahydro-1,3,4- } \\
\text { metheno-2H-cyclobuta[cd]pentalen- } \\
\text { 2-one }^{\text {b }}\end{array}$ & chiral \\
\hline 44 & 3 & & $\begin{array}{l}\text { 1,2,4,6,7,9,10- } \\
\left.\text { heptachloropentacyclo[5.3.0.0.0 } 0^{2,6} \cdot 0^{3,9} \cdot 0^{4,8}\right] \text { decan-5-one }\end{array}$ & $\begin{array}{l}\text { 1,1a,3,4,5,5a,5b- } \\
\text { heptachlorooctahydro-1,3,4- } \\
\text { metheno-2H-cyclobuta[cd]pentalen- } \\
\text { 2-one }^{\text {b }}\end{array}$ & chiral \\
\hline 45 & 3 & & $\begin{array}{l}\text { 1,r-2,3,4,8,9,c-10- } \\
\left.\text { heptachloropentacyclo[5.3.0.0 } 0^{2,6} \cdot 0^{3,9} \cdot 0^{4,8}\right] \text { decan-5-one }\end{array}$ & $\begin{array}{l}r-1,1 \mathrm{a}, 4, c-5,5 \mathrm{a}, 5 \mathrm{~b}, 6- \\
\text { heptachlorooctahydro-1,3,4- } \\
\text { metheno-2H-cyclobuta[cd]pentalen- } \\
\text { 2-one }\end{array}$ & chiral \\
\hline 46 & 3 & & $\begin{array}{l}\text { 1,r-2,3,4,8,9,t-10- } \\
\left.\text { heptachloropentacyclo[5.3.0.0.0 } 0^{2,6} \cdot 0^{3,9} \cdot 0^{4,8}\right] \text { decan-5-one }\end{array}$ & $\begin{array}{l}r-1,1 \mathrm{a}, 4, t-5,5 \mathrm{a}, 5 \mathrm{~b}, 6- \\
\text { heptachlorooctahydro-1,3,4- } \\
\text { metheno-2H-cyclobuta[cd]pentalen- } \\
\text { 2-one }\end{array}$ & chiral \\
\hline 47 & 3 & & $\begin{array}{l}\text { 2,3,4,6,7,10,10- } \\
\left.\text { heptachloropentacyclo[5.3.0.0.0 } 0^{2,6} \cdot 0^{3,9} \cdot 0^{4,8}\right] \text { decan-5-one }\end{array}$ & $\begin{array}{l}\text { 1,1a,3,3a,5,5,6- } \\
\text { heptachlorooctahydro-1,3,4- } \\
\text { metheno-2H-cyclobuta[cd]pentalen- } \\
\text { 2-one }\end{array}$ & chiral \\
\hline
\end{tabular}


Gibbs Free Energy of Formation of Chlordecone and Potential Degradation Products: Implications for Remediation Strategies and Environmental Fate Table SI-1 Structures of all chlordecone potential dechlorinated congeners and their IUPAC and CAS names

\begin{tabular}{|c|c|c|c|c|c|}
\hline Entry & $\overline{\mathrm{nCl}}$ & Structure $^{a}$ & IUPAC - Name & CAS - Name & Chirality \\
\hline 48 & 3 & & $\begin{array}{l}1,3,4,6,7,10,10- \\
\left.\text { heptachloropentacyclo[5.3.0.0 } 0^{2,6} \cdot 0^{3,9} \cdot 0^{4,8}\right] \text { decan-5-one }\end{array}$ & $\begin{array}{l}\text { 1,1a,3,3a,4,5,5- } \\
\text { heptachlorooctahydro-1,3,4- } \\
\text { metheno-2H-cyclobuta[cd]pentalen- } \\
\text { 2-one }\end{array}$ & chiral \\
\hline 49 & 3 & & $\begin{array}{l}1,2,3,4,7,10,10- \\
\left.\text { heptachloropentacyclo[5.3.0.0 } 0^{2,6} \cdot 0^{3,9} \cdot 0^{4,8}\right] \text { decan-5-one }\end{array}$ & $\begin{array}{l}\text { 1,1a,3a,4,5,5,6- } \\
\text { heptachlorooctahydro-1,3,4- } \\
\text { metheno-2H-cyclobuta[cd]pentalen- } \\
\text { 2-one }\end{array}$ & chiral \\
\hline 50 & 3 & & $\begin{array}{l}1,2,3,4,6,10,10- \\
\left.\text { heptachloropentacyclo[5.3.0.0 } 0^{2,6} \cdot 0^{3,9} \cdot 0^{4,8}\right] \text { decan-5-one }\end{array}$ & $\begin{array}{l}\text { 1,1a,3,4,5,5,6- } \\
\text { heptachlorooctahydro-1,3,4- } \\
\text { metheno-2H-cyclobuta[cd]pentalen- } \\
\text { 2-one }\end{array}$ & chiral \\
\hline 51 & 3 & & $\begin{array}{l}\text { 1,3,6,7,8,10,10- } \\
\left.\text { heptachloropentacyclo[5.3.0.0 } 0^{2,6} \cdot 0^{3,9} \cdot 0^{4,8}\right] \text { decan-5-one }\end{array}$ & $\begin{array}{l}\text { 1,3,3a,4,5,5,5b- } \\
\text { heptachlorooctahydro-1,3,4- } \\
\text { metheno-2H-cyclobuta[cd]pentalen- } \\
\text { 2-one }\end{array}$ & chiral \\
\hline 52 & 3 & & $\begin{array}{l}\text { 1,2,4,6,9,10,10- } \\
\left.\text { heptachloropentacyclo[5.3.0.0 } 0^{2,6} \cdot 0^{3,9} \cdot 0^{4,8}\right] \text { decan-5-one }\end{array}$ & $\begin{array}{l}\text { 1,1a,3,4,5,5,5a- } \\
\text { heptachlorooctahydro-1,3,4- } \\
\text { metheno-2H-cyclobuta[cd]pentalen- } \\
\text { 2-one } \text { b }^{\text {b }}\end{array}$ & chiral \\
\hline 53 & 3 & & $\begin{array}{l}\text { 1,2,3,4,8,10,10- } \\
\left.\text { heptachloropentacyclo[5.3.0.0.0 } 0^{2,6} \cdot 0^{3,9} \cdot 0^{4,8}\right] \text { decan-5-one }\end{array}$ & $\begin{array}{l}\text { 1,1a,4,5,5,5b,6- } \\
\text { heptachlorooctahydro-1,3,4- } \\
\text { metheno-2H-cyclobuta[cd]pentalen- } \\
\text { 2-one }\end{array}$ & chiral \\
\hline 54 & 3 & H & $\begin{array}{l}1,2,6,8,9,10,10- \\
\left.\text { heptachloropentacyclo[5.3.0.0 } 0^{2,6} \cdot 0^{3,9} \cdot 0^{4,8}\right] \text { decan-5-one }\end{array}$ & $\begin{array}{l}\text { 1,1a,3a,4,5,5,5a- } \\
\text { heptachlorooctahydro-1,3,4- } \\
\text { metheno-2H-cyclobuta[cd]pentalen- } \\
\text { 2-one } \text { b }^{\text {- }}\end{array}$ & chiral \\
\hline
\end{tabular}


Gibbs Free Energy of Formation of Chlordecone and Potential Degradation Products: Implications for Remediation Strategies and Environmental Fate Table SI-1 Structures of all chlordecone potential dechlorinated congeners and their IUPAC and CAS names

\begin{tabular}{|c|c|c|c|c|c|}
\hline Entry & $\overline{\mathrm{nCl}}$ & Structure $^{a}$ & IUPAC - Name & CAS - Name & Chirality \\
\hline 55 & 3 & & $\begin{array}{l}1,2,3,7,9,10,10- \\
\left.\text { heptachloropentacyclo[5.3.0.0 } 0^{2,6} \cdot 0^{3,9} \cdot 0^{4,8}\right] \text { decan-5-one }\end{array}$ & $\begin{array}{l}\text { 1,3a,4,5,5,5a,5b- } \\
\text { heptachlorooctahydro-1,3,4- } \\
\text { metheno-2H-cyclobuta[cd]pentalen- } \\
\text { 2-onec }\end{array}$ & chiral \\
\hline 56 & 3 & & $\begin{array}{l}1,2,3,4,9,10,10- \\
\left.\text { heptachloropentacyclo[5.3.0.0 } 0^{2,6} \cdot 0^{3,9} \cdot 0^{4,8}\right] \text { decan-5-one }\end{array}$ & $\begin{array}{l}\text { 1,1a,4,5,5,5a,6- } \\
\text { heptachlorooctahydro-1,3,4- } \\
\text { metheno-2H-cyclobuta[cd]pentalen- } \\
\text { 2-one }\end{array}$ & chiral \\
\hline 57 & 3 & & $\begin{array}{l}1,2,3,6,7,10,10- \\
\left.\text { heptachloropentacyclo[5.3.0.0 } 0^{2,6} \cdot 0^{3,9} \cdot 0^{4,8}\right] \text { decan-5-one }\end{array}$ & $\begin{array}{l}\text { 1,1a,3a,5,5,5a,5b- } \\
\text { heptachlorooctahydro-1,3,4- } \\
\text { metheno-2H-cyclobuta[cd]pentalen- } \\
\text { 2-onec }\end{array}$ & chiral \\
\hline 58 & 4 & & $\begin{array}{l}1,2,3,6,7,8- \\
\left.\text { hexachloropentacyclo[5.3.0.0 } 0^{2,6} \cdot 0^{3,9} \cdot 0^{4,8}\right] \text { decan-5-one }\end{array}$ & $\begin{array}{l}1,1 \mathrm{a}, 3 \mathrm{a}, 5 \mathrm{a}, 5 \mathrm{~b}, 6- \\
\text { hexachlorooctahydro-1,3,4- } \\
\text { metheno-2H-cyclobuta[cd]pentalen- } \\
\text { 2-one }^{\text {b }}\end{array}$ & meso \\
\hline 59 & 4 & & $\begin{array}{l}1,2,4,6,8,9- \\
\left.\text { hexachloropentacyclo[5.3.0.0 } 0^{2,6} \cdot 0^{3,9} \cdot 0^{4,8}\right] \text { decan-5-one }\end{array}$ & $\begin{array}{l}\text { 1,1a,3,4,5a,5b- } \\
\text { hexachlorooctahydro-1,3,4- } \\
\text { metheno-2H-cyclobuta[cd]pentalen- } \\
\text { 2-one }{ }^{\text {b }}\end{array}$ & meso \\
\hline 60 & 4 & & $\begin{array}{l}2,3,4,6,7,8- \\
\left.\text { hexachloropentacyclo[5.3.0.0 } 0^{2,6} \cdot 0^{3,9} \cdot 0^{4,8}\right] \text { decan-5-one }\end{array}$ & $\begin{array}{l}\text { 1,1a,3,3a,5b,6- } \\
\text { hexachlorooctahydro-1,3,4- } \\
\text { metheno-2H-cyclobuta[cd]pentalen- } \\
\text { 2-one }\end{array}$ & meso \\
\hline
\end{tabular}


Gibbs Free Energy of Formation of Chlordecone and Potential Degradation Products: Implications for Remediation Strategies and Environmental Fate Table SI-1 Structures of all chlordecone potential dechlorinated congeners and their IUPAC and CAS names

\begin{tabular}{|c|c|c|c|c|c|}
\hline Entry & $\overline{\mathrm{nCl}}$ & Structure $^{a}$ & IUPAC - Name & CAS - Name & Chirality \\
\hline 61 & 4 & H & $\begin{array}{l}1,2,3,4,6,9- \\
\left.\text { hexachloropentacyclo[5.3.0.0 } 0^{2,6} \cdot 0^{3,9} \cdot 0^{4,8}\right] \text { decan-5-one }\end{array}$ & $\begin{array}{l}\text { 1,1a,3,4,5a,6-hexachlorooctahydro- } \\
\text { 1,3,4-metheno-2H- } \\
\text { cyclobuta[cd]pentalen-2-one }\end{array}$ & meso \\
\hline 62 & 4 & & $\begin{array}{l}1,2,3,7,8,9- \\
\left.\text { hexachloropentacyclo[5.3.0.0 } 0^{2,6} \cdot 0^{3,9} \cdot 0^{4,8}\right] \text { decan-5-one }\end{array}$ & $\begin{array}{l}\text { 1,3a,4,5a,5b,6- } \\
\text { hexachlorooctahydro-1,3,4- } \\
\text { metheno-2H-cyclobuta[cd]pentalen- } \\
\text { 2-one }\end{array}$ & meso \\
\hline 63 & 4 & & $\begin{array}{l}1,2,3,4,7,8- \\
\left.\text { hexachloropentacyclo[5.3.0.0 } 0^{2,6} \cdot 0^{3,9} \cdot 0^{4,8}\right] \text { decan-5-one }\end{array}$ & $\begin{array}{l}\text { 1,1a,3a,4,5b,6- } \\
\text { hexachlorooctahydro-1,3,4- } \\
\text { metheno-2H-cyclobuta[cd]pentalen- } \\
\text { 2-one }\end{array}$ & meso \\
\hline 64 & 4 & & $\begin{array}{l}1,2,7,9,10,10- \\
\left.\text { hexachloropentacyclo[5.3.0.0 } 0^{2,6} \cdot 0^{3,9} \cdot 0^{4,8}\right] \text { decan-5-one }\end{array}$ & $\begin{array}{l}\text { 1,4,5,5,5a,5b-hexachlorooctahydro- } \\
\text { 1,3,4-metheno-2H- } \\
\text { cyclobuta[cd]pentalen-2-one }^{\mathrm{b}}\end{array}$ & meso \\
\hline 65 & 4 & & $\begin{array}{l}2,3,7,8,10,10- \\
\left.\text { hexachloropentacyclo[5.3.0.0 } 0^{2,6} \cdot 0^{3,9} \cdot 0^{4,8}\right] \text { decan-5-one }\end{array}$ & $\begin{array}{l}\text { 1,3a,5,5,5b,6-hexachlorooctahydro- } \\
\text { 1,3,4-metheno-2H- } \\
\text { cyclobuta[cd]pentalen-2-one }\end{array}$ & meso \\
\hline 66 & 4 & & $\begin{array}{l}1,3,6,8,10,10- \\
\left.\text { hexachloropentacyclo[5.3.0.0 } 0^{2,6} \cdot 0^{3,9} \cdot 0^{4,8}\right] \text { decan-5-one }\end{array}$ & $\begin{array}{l}\text { 1,3,4,5,5,5b-hexachlorooctahydro- } \\
\text { 1,3,4-metheno-2H- } \\
\text { cyclobuta[cd]pentalen-2-one }\end{array}$ & meso \\
\hline 67 & 4 & & $\begin{array}{l}1,2,3,9,10,10- \\
\left.\text { hexachloropentacyclo[5.3.0.0 } 0^{2,6} \cdot 0^{3,9} \cdot 0^{4,8}\right] \text { decan-5-one }\end{array}$ & $\begin{array}{l}\text { 1,4,5,5,5a,6-hexachlorooctahydro- } \\
\text { 1,3,4-metheno-2H- } \\
\text { cyclobuta[cd]pentalen-2-one }\end{array}$ & meso \\
\hline
\end{tabular}


Gibbs Free Energy of Formation of Chlordecone and Potential Degradation Products: Implications for Remediation Strategies and Environmental Fate Table SI-1 Structures of all chlordecone potential dechlorinated congeners and their IUPAC and CAS names

\begin{tabular}{|c|c|c|c|c|c|}
\hline Entry & $\overline{\mathrm{nCl}}$ & Structure $^{a}$ & IUPAC - Name & CAS - Name & Chirality \\
\hline 68 & 4 & & $\begin{array}{l}1,2,6,7,10,10- \\
\left.\text { hexachloropentacyclo[5.3.0.0 } 0^{2,6} \cdot 0^{3,9} \cdot 0^{4,8}\right] \text { decan-5-one }\end{array}$ & $\begin{array}{l}\text { 1,1a,5,5,5a,5b- } \\
\text { hexachlorooctahydro-1,3,4- } \\
\text { metheno-2H-cyclobuta[cd]pentalen- } \\
\text { 2-one } \text { b }^{\text {- }}\end{array}$ & meso \\
\hline 69 & 4 & & $\begin{array}{l}1,2,4,7,10,10- \\
\left.\text { hexachloropentacyclo[5.3.0.0 } 0^{2,6} \cdot 0^{3,9} \cdot 0^{4,8}\right] \text { decan-5-one }\end{array}$ & $\begin{array}{l}\text { 1,3,5,5,5a,5b-hexachlorooctahydro- } \\
\text { 1,3,4-metheno- } \mathrm{H} \text { - } \\
\text { cyclobuta[cd]pentalen-2-one }^{\mathrm{b}}\end{array}$ & meso \\
\hline 70 & 4 & & $\begin{array}{l}1,3,4,8,10,10- \\
\left.\text { hexachloropentacyclo[5.3.0.0 } 0^{2,6} \cdot 0^{3,9} \cdot 0^{4,8}\right] \text { decan-5-one }\end{array}$ & $\begin{array}{l}\text { 1,1a,4,5,5,5b-hexachlorooctahydro- } \\
\text { 1,3,4-metheno- } 2 \mathrm{H} \text { - } \\
\text { cyclobuta[cd]pentalen-2-one }\end{array}$ & meso \\
\hline 71 & 4 & & $\begin{array}{l}2,4,6,7,10,10- \\
\left.\text { hexachloropentacyclo[5.3.0.0 } 0^{2,6} \cdot 0^{3,9} \cdot 0^{4,8}\right] \text { decan-5-one }\end{array}$ & $\begin{array}{l}\text { 1,1a,3,5,5,5b-hexachlorooctahydro- } \\
\text { 1,3,4-metheno- } 2 \mathrm{H}- \\
\text { cyclobuta[cd]pentalen-2-one }^{\mathrm{b}}\end{array}$ & meso \\
\hline 72 & 4 & & $\begin{array}{l}2,3,4,6,10,10- \\
\left.\text { hexachloropentacyclo[5.3.0.0 } 0^{2,6} \cdot 0^{3,9} \cdot 0^{4,8}\right] \text { decan-5-one }\end{array}$ & $\begin{array}{l}\text { 1,1a,3,5,5,6-hexachlorooctahydro- } \\
\text { 1,3,4-metheno-2H- } \\
\text { cyclobuta[cd]pentalen-2-one }\end{array}$ & meso \\
\hline 73 & 4 & & $\begin{array}{l}1,2,4,6,7,8- \\
\left.\text { hexachloropentacyclo[5.3.0.0 } 0^{2,6} \cdot 0^{3,9} \cdot 0^{4,8}\right] \text { decan-5-one }\end{array}$ & $\begin{array}{l}\text { 1,1a,3,3a,5a,5b- } \\
\text { hexachlorooctahydro-1,3,4- } \\
\text { metheno-2H-cyclobuta[cd]pentalen- } \\
\text { 2-one }^{\text {b }}\end{array}$ & chiral \\
\hline 74 & 4 & & $\begin{array}{l}1,2,6,7,8,9- \\
\left.\text { hexachloropentacyclo[5.3.0.0 } 0^{2,6} \cdot 0^{3,9} \cdot 0^{4,8}\right] \text { decan-5-one }\end{array}$ & $\begin{array}{l}\text { 1,1a,3a,4,5a,5b- } \\
\text { hexachlorooctahydro-1,3,4- } \\
\text { metheno-2H-cyclobuta[cd]pentalen- } \\
\text { 2-one } \text { b }^{\text {- }}\end{array}$ & chiral \\
\hline
\end{tabular}


Gibbs Free Energy of Formation of Chlordecone and Potential Degradation Products: Implications for Remediation Strategies and Environmental Fate Table SI-1 Structures of all chlordecone potential dechlorinated congeners and their IUPAC and CAS names

\begin{tabular}{|c|c|c|c|c|c|}
\hline Entry & $\overline{\mathrm{nCl}}$ & Structure $^{a}$ & IUPAC - Name & CAS - Name & Chirality \\
\hline 75 & 4 & $\overrightarrow{\mathbf{H}}$ & $\begin{array}{l}1,3,4,6,7,8- \\
\left.\text { hexachloropentacyclo[5.3.0.0 } 0^{2,6} \cdot 0^{3,9} \cdot 0^{4,8}\right] \text { decan-5-one }\end{array}$ & $\begin{array}{l}\text { 1,1a,3,3a,4,5b- } \\
\text { hexachlorooctahydro-1,3,4- } \\
\text { metheno-2H-cyclobuta[cd]pentalen- } \\
\text { 2-one }\end{array}$ & chiral \\
\hline 76 & 4 & H & $\begin{array}{l}1,2,4,7,8,9- \\
\left.\text { hexachloropentacyclo[5.3.0.0 } 0^{2,6} \cdot 0^{3,9} \cdot 0^{4,8}\right] \text { decan-5-one }\end{array}$ & $\begin{array}{l}\text { 1,3,3a,4,5a,5b- } \\
\text { hexachlorooctahydro-1,3,4- } \\
\text { metheno-2H-cyclobuta[cd]pentalen- } \\
\text { 2-one }{ }^{\text {b }}\end{array}$ & chiral \\
\hline 77 & 4 & & $\begin{array}{l}1,2,4,6,8,9- \\
\left.\text { hexachloropentacyclo[5.3.0.0 } 0^{2,6} \cdot 0^{3,9} \cdot 0^{4,8}\right] \text { decan-5-one }\end{array}$ & $\begin{array}{l}\text { 1,1a,3,3a,4,5a,- } \\
\text { hexachlorooctahydro-1,3,4- } \\
\text { metheno-2H-cyclobuta[cd]pentalen- } \\
\text { 2-one }{ }^{\text {b }}\end{array}$ & chiral \\
\hline 78 & 4 & & $\begin{array}{l}1, r-2,3,6,7, t-10- \\
\left.\text { hexachloropentacyclo[5.3.0.0 } 0^{2,6} \cdot 0^{3,9} \cdot 0^{4,8}\right] \text { decan-5-one }\end{array}$ & $\begin{array}{l}r-1,1 \mathrm{a}, t-5,5 \mathrm{a}, 5 \mathrm{~b}, 6- \\
\text { hexachlorooctahydro-1,3,4- } \\
\text { metheno-2H-cyclobuta[cd]pentalen- } \\
\text { 2-one }{ }^{\mathrm{b}}\end{array}$ & chiral \\
\hline 79 & 4 & & $\begin{array}{l}1, r-2,3,6,7, c-10- \\
\left.\text { hexachloropentacyclo[5.3.0.0 } 0^{2,6} \cdot 0^{3,9} \cdot 0^{4,8}\right] \text { decan-5-one }\end{array}$ & $\begin{array}{l}r-1,1 \mathrm{a}, c-5,5 \mathrm{a}, 5 \mathrm{~b}, 6- \\
\text { hexachlorooctahydro-1,3,4- } \\
\text { metheno-2H-cyclobuta[cd]pentalen- } \\
\text { 2-one }\end{array}$ & chiral \\
\hline 80 & 4 & & $\begin{array}{l}1,2,6,7,9,10- \\
\left.\text { hexachloropentacyclo[5.3.0.0 } 0^{2,6} \cdot 0^{3,9} \cdot 0^{4,8}\right] \text { decan-5-one }\end{array}$ & $\begin{array}{l}\text { 1,1a,4,5,5a,5b- } \\
\text { hexachlorooctahydro-1,3,4- } \\
\text { metheno-2H-cyclobuta[cd]pentalen- } \\
\text { 2-one }\end{array}$ & chiral \\
\hline
\end{tabular}


Gibbs Free Energy of Formation of Chlordecone and Potential Degradation Products: Implications for Remediation Strategies and Environmental Fate Table SI-1 Structures of all chlordecone potential dechlorinated congeners and their IUPAC and CAS names

\begin{tabular}{|c|c|c|c|c|c|}
\hline Entry & $\overline{\mathrm{nCl}}$ & Structure $^{a}$ & IUPAC - Name & CAS - Name & Chirality \\
\hline 81 & 4 & & $\begin{array}{l}1,2,4,6,7,10- \\
\left.\text { hexachloropentacyclo[5.3.0.0 } 0^{2,6} \cdot 0^{3,9} \cdot 0^{4,8}\right] \text { decan-5-one }\end{array}$ & $\begin{array}{l}\text { 1,1a,3,5,5a,5b- } \\
\text { hexachlorooctahydro-1,3,4- } \\
\text { metheno-2H-cyclobuta[cd]pentalen- } \\
\text { 2-one }{ }^{\text {b }}\end{array}$ & chiral \\
\hline 82 & 4 & & $\begin{array}{l}r-2,3,4,6,8, t-10- \\
\text { hexachloropentacyclo[5.3.0.0 } \\
\left.2,6 \cdot 0^{3,9} \cdot 0^{4,8}\right] \text { decan-5-one }\end{array}$ & $\begin{array}{l}r-1,1 \mathrm{a}, 3, t-5,5 \mathrm{~b}, 6- \\
\text { hexachlorooctahydro-1,3,4- } \\
\text { metheno-2H-cyclobuta[cd]pentalen- } \\
\text { 2-one }\end{array}$ & chiral \\
\hline 83 & 4 & & $\begin{array}{l}r-2,3,4,6,8, c-10- \\
\left.\text { hexachloropentacyclo[5.3.0.0 } 0^{2,6} \cdot 0^{3,9} \cdot 0^{4,8}\right] \text { decan-5-one }\end{array}$ & $\begin{array}{l}r-1,1 \mathrm{a}, 3, c-5,5 \mathrm{~b}, 6- \\
\text { hexachlorooctahydro-1,3,4- } \\
\text { metheno-2H-cyclobuta[cd]pentalen- } \\
\text { 2-one }\end{array}$ & chiral \\
\hline 84 & 4 & & $\begin{array}{l}1, r-2,3,4,6, t-10- \\
\left.\text { hexachloropentacyclo[5.3.0.0 } 0^{2,6} \cdot 0^{3,9} \cdot 0^{4,8}\right] \text { decan-5-one }\end{array}$ & $\begin{array}{l}r-1,1 \mathrm{a}, 3,4, t-5,6- \\
\text { hexachlorooctahydro-1,3,4- } \\
\text { metheno-2H-cyclobuta[cd]pentalen- } \\
\text { 2-one }\end{array}$ & chiral \\
\hline 85 & 4 & & $\begin{array}{l}1, r-2,3,4,6, c-10- \\
\left.\text { hexachloropentacyclo[5.3.0.0 } 0^{2,6} \cdot 0^{3,9} \cdot 0^{4,8}\right] \text { decan-5-one }\end{array}$ & $\begin{array}{l}r-1,1 \mathrm{a}, 3,4, c-5,6- \\
\text { hexachlorooctahydro-1,3,4- } \\
\text { metheno-2H-cyclobuta[cd]pentalen- } \\
\text { 2-one }\end{array}$ & chiral \\
\hline 86 & 4 & & $\begin{array}{l}1, r-2,3,4,7, t-10- \\
\left.\text { hexachloropentacyclo[5.3.0.0 } 0^{2,6} \cdot 0^{3,9} \cdot 0^{4,8}\right] \text { decan-5-one }\end{array}$ & $\begin{array}{l}r-1,1 \mathrm{a}, 3 \mathrm{a}, 4, t-5,6- \\
\text { hexachlorooctahydro-1,3,4- } \\
\text { metheno-2H-cyclobuta[cd]pentalen- } \\
\text { 2-one }\end{array}$ & chiral \\
\hline 87 & 4 & & $\begin{array}{l}1, r-2,3,4,7, c-10- \\
\left.\text { hexachloropentacyclo[5.3.0.0 } 0^{2,6} \cdot 0^{3,9} \cdot 0^{4,8}\right] \text { decan-5-one }\end{array}$ & $\begin{array}{l}r-1,1 \mathrm{a}, 3 \mathrm{a}, 4, c-5,6- \\
\text { hexachlorooctahydro-1,3,4- } \\
\text { metheno-2H-cyclobuta[cd]pentalen- } \\
\text { 2-one }\end{array}$ & chiral \\
\hline
\end{tabular}


Gibbs Free Energy of Formation of Chlordecone and Potential Degradation Products: Implications for Remediation Strategies and Environmental Fate Table SI-1 Structures of all chlordecone potential dechlorinated congeners and their IUPAC and CAS names

\begin{tabular}{|c|c|c|c|c|c|}
\hline Entry & $\overline{\mathrm{nCl}}$ & Structure $^{a}$ & IUPAC - Name & CAS - Name & Chirality \\
\hline 88 & 4 & & $\begin{array}{l}1, r-2,4,6,8, c-10- \\
\left.\text { hexachloropentacyclo[5.3.0.0 } 0^{2,6} \cdot 0^{3,9} \cdot 0^{4,8}\right] \text { decan-5-one }\end{array}$ & $\begin{array}{l}r-1,1 \mathrm{a}, 3,3 \mathrm{a}, c-5,5 \mathrm{a}- \\
\text { hexachlorooctahydro-1,3,4- } \\
\text { metheno-2H-cyclobuta[cd]pentalen- } \\
\text { 2-one }^{\mathrm{b}}\end{array}$ & chiral \\
\hline 89 & 4 & & $\begin{array}{l}1, r-2,4,6,8, t-10- \\
\left.\text { hexachloropentacyclo[5.3.0.0 } 0^{2,6} \cdot 0^{3,9} \cdot 0^{4,8}\right] \text { decan-5-one }\end{array}$ & $\begin{array}{l}r-1,1 \mathrm{a}, 3,3 \mathrm{a}, t-5,5 \mathrm{a}- \\
\text { hexachlorooctahydro-1,3,4- } \\
\text { metheno-2H-cyclobuta[cd]pentalen- } \\
\text { 2-one }^{\mathrm{b}}\end{array}$ & chiral \\
\hline 90 & 4 & & $\begin{array}{l}1, r-2,3,4,8, t-10- \\
\left.\text { hexachloropentacyclo[5.3.0.0 } 0^{2,6} \cdot 0^{3,9} \cdot 0^{4,8}\right] \text { decan-5-one }\end{array}$ & $\begin{array}{l}r-1,1 \mathrm{a}, 3 \mathrm{a}, 4, c-5,5 \mathrm{~b}- \\
\text { hexachlorooctahydro-1,3,4- } \\
\text { metheno-2H-cyclobuta[cd]pentalen- } \\
\text { 2-one }{ }^{\mathbf{b}, \mathrm{c}}\end{array}$ & chiral \\
\hline 91 & 4 & & $\begin{array}{l}1, r-2,3,4,8, c-10- \\
\left.\text { hexachloropentacyclo[5.3.0.0 } 0^{2,6} \cdot 0^{3,9} \cdot 0^{4,8}\right] \text { decan-5-one }\end{array}$ & $\begin{array}{l}r-1,1 \mathrm{a}, 4, c-5,5 \mathrm{~b}, 6- \\
\text { hexachlorooctahydro-1,3,4- } \\
\text { metheno-2H-cyclobuta[cd]pentalen- } \\
\text { 2-one }\end{array}$ & chiral \\
\hline 92 & 4 & & $\begin{array}{l}1, r-2,3,4,9, t-10- \\
\left.\text { hexachloropentacyclo[5.3.0.0 } 0^{2,6} \cdot 0^{3,9} \cdot 0^{4,8}\right] \text { decan-5-one }\end{array}$ & $\begin{array}{l}r-1,1 \mathrm{a}, 4, t-5,5 \mathrm{a}, 6- \\
\text { hexachlorooctahydro-1,3,4- } \\
\text { metheno-2H-cyclobuta[cd]pentalen- } \\
\text { 2-one }\end{array}$ & chiral \\
\hline 93 & 4 & & $\begin{array}{l}1, r-2,3,4,9, c-10- \\
\left.\text { hexachloropentacyclo[5.3.0.0 } 0^{2,6} \cdot 0^{3,9} \cdot 0^{4,8}\right] \text { decan-5-one }\end{array}$ & $\begin{array}{l}r-1,1 \mathrm{a}, 4, c-5,5 \mathrm{a}, 6- \\
\text { hexachlorooctahydro-1,3,4- } \\
\text { metheno-2H-cyclobuta[cd]pentalen- } \\
\text { 2-one }\end{array}$ & chiral \\
\hline
\end{tabular}


Gibbs Free Energy of Formation of Chlordecone and Potential Degradation Products: Implications for Remediation Strategies and Environmental Fate Table SI-1 Structures of all chlordecone potential dechlorinated congeners and their IUPAC and CAS names

\begin{tabular}{|c|c|c|c|c|c|}
\hline Entry & $\overline{\mathrm{nCl}}$ & Structure $^{a}$ & IUPAC - Name & CAS - Name & Chirality \\
\hline 94 & 4 & $\mathbf{H}_{\mathbf{U}}$ & $\begin{array}{l}1, r-2,3,7,9, t-10- \\
\left.\text { hexachloropentacyclo[5.3.0.0 } 0^{2,6} \cdot 0^{3,9} \cdot 0^{4,8}\right] \text { decan-5-one }\end{array}$ & $\begin{array}{l}r-1,3 a, 4, c-5,5 a, 5 b- \\
\text { hexachlorooctahydro-1,3,4- } \\
\text { metheno-2H-cyclobuta[cd]pentalen- } \\
\text { 2-one }{ }^{c}\end{array}$ & chiral \\
\hline 95 & 4 & & $\begin{array}{l}1, r-2,3,7,9, c-10- \\
\left.\text { hexachloropentacyclo[5.3.0.0 } 0^{2,6} \cdot 0^{3,9} \cdot 0^{4,8}\right] \text { decan-5-one }\end{array}$ & $\begin{array}{l}r-1,3 a, 4, t-5,5 a, 5 b- \\
\text { hexachlorooctahydro-1,3,4- } \\
\text { metheno-2H-cyclobuta[cd]pentalen- } \\
\text { 2-one }{ }^{c}\end{array}$ & chiral \\
\hline 96 & 4 & & $\begin{array}{l}1, r-2,4,8,9, t-10- \\
\left.\text { hexachloropentacyclo[5.3.0.0 } 0^{2,6} \cdot 0^{3,9} \cdot 0^{4,8}\right] \text { decan-5-one }\end{array}$ & $\begin{array}{l}r-1,1 \mathrm{a}, 3 \mathrm{a}, 4, c-5,5 \mathrm{a}- \\
\text { hexachlorooctahydro-1,3,4- } \\
\text { metheno-2H-cyclobuta[cd]pentalen- } \\
\text { 2-one } \text { b,c }^{\text {b.c }}\end{array}$ & chiral \\
\hline 97 & 4 & & $\begin{array}{l}1, r-2,4,8,9, c-10- \\
\left.\text { hexachloropentacyclo[5.3.0.0 } 0^{2,6} \cdot 0^{3,9} \cdot 0^{4,8}\right] \text { decan-5-one }\end{array}$ & $\begin{array}{l}r-1,1 \mathrm{a}, 3,3 \mathrm{a}, 4, t-5,5 \mathrm{a}- \\
\text { hexachlorooctahydro-1,3,4- } \\
\text { metheno-2H-cyclobuta[cd]pentalen- } \\
\text { 2-one } \text { b,c }^{\text {b. }}\end{array}$ & chiral \\
\hline 98 & 4 & & $\begin{array}{l}1,3,4,6,8,10- \\
\left.\text { hexachloropentacyclo[5.3.0.0 } 0^{2,6} \cdot 0^{3,9} \cdot 0^{4,8}\right] \text { decan-5-one }\end{array}$ & $\begin{array}{l}\text { 1,1a,3,4,5,5b-hexachlorooctahydro- } \\
\text { 1,3,4-metheno- } 2 \mathrm{H}- \\
\text { cyclobuta[cd]pentalen-2-one }\end{array}$ & chiral \\
\hline 99 & 4 & & $\begin{array}{l}1, r-2,4,6,9, t-10- \\
\left.\text { hexachloropentacyclo[5.3.0.0 } 0^{2,6} \cdot 0^{3,9} \cdot 0^{4,8}\right] \text { decan-5-one }\end{array}$ & $\begin{array}{l}r-1,1 \mathrm{a}, 3,4, t-5,5 \mathrm{a}- \\
\text { hexachlorooctahydro-1,3,4- } \\
\text { metheno-2H-cyclobuta[cd]pentalen- } \\
\text { 2-one } \text { b }^{\text {b }}\end{array}$ & chiral \\
\hline 100 & 4 & & $\begin{array}{l}1, r-2,4,6,9, c-10- \\
\left.\text { hexachloropentacyclo[5.3.0.0 } 0^{2,6} \cdot 0^{3,9} \cdot 0^{4,8}\right] \text { decan-5-one }\end{array}$ & $\begin{array}{l}r-1,1 \mathrm{a}, 3,4, c-5,5 \mathrm{a}- \\
\text { hexachlorooctahydro-1,3,4- } \\
\text { metheno-2H-cyclobuta[cd]pentalen- } \\
\text { 2-one } \text { b }^{\text {b }}\end{array}$ & chiral \\
\hline
\end{tabular}


Gibbs Free Energy of Formation of Chlordecone and Potential Degradation Products: Implications for Remediation Strategies and Environmental Fate Table SI-1 Structures of all chlordecone potential dechlorinated congeners and their IUPAC and CAS names

\begin{tabular}{|c|c|c|c|c|c|}
\hline Entry & $\overline{\mathrm{nCl}}$ & Structure $^{a}$ & IUPAC - Name & CAS - Name & Chirality \\
\hline 101 & 4 & & $\begin{array}{l}1,2,4,7,9,10- \\
\left.\text { hexachloropentacyclo[5.3.0.0 } 0^{2,6} \cdot 0^{3,9} \cdot 0^{4,8}\right] \text { decan-5-one }\end{array}$ & $\begin{array}{l}\text { 1,3,4,5,5a,5b-hexachlorooctahydro- } \\
\text { 1,3,4-metheno- } \mathrm{H} \text { - } \\
\text { cyclobuta[cd]pentalen-2-one }^{\mathbf{b}}\end{array}$ & chiral \\
\hline 102 & 4 & & $\begin{array}{l}2,3,4,7,8,10- \\
\left.\text { hexachloropentacyclo[5.3.0.0 } 0^{2,6} \cdot 0^{3,9} \cdot 0^{4,8}\right] \text { decan-5-one }\end{array}$ & $\begin{array}{l}\text { 1,1a,3a,5,5b,6- } \\
\text { hexachlorooctahydro-1,3,4- } \\
\text { metheno-2H-cyclobuta[cd]pentalen- } \\
\text { 2-one }\end{array}$ & chiral \\
\hline 103 & 4 & & $\begin{array}{l}1, r-2,3,6,8, t-10- \\
\left.\text { hexachloropentacyclo[5.3.0.0 } 0^{2,6} \cdot 0^{3,9} \cdot 0^{4,8}\right] \text { decan-5-one }\end{array}$ & $\begin{array}{l}r-1,1 \mathrm{a}, 3 \mathrm{a}, t-5,5 \mathrm{a}, 6- \\
\text { hexachlorooctahydro-1,3,4- } \\
\text { metheno-2H-cyclobuta[cd]pentalen- } \\
\text { 2-one }^{\text {b }}\end{array}$ & chiral \\
\hline 104 & 4 & & $\begin{array}{l}1, r-2,3,6,8, c-10- \\
\left.\text { hexachloropentacyclo[5.3.0.0 } 0^{2,6} \cdot 0^{3,9} \cdot 0^{4,8}\right] \text { decan-5-one }\end{array}$ & $\begin{array}{l}r-1,1 \mathrm{a}, 3 \mathrm{a}, c-5,5 \mathrm{a}, 6- \\
\text { hexachlorooctahydro-1,3,4- } \\
\text { metheno-2H-cyclobuta[cd]pentalen- } \\
\text { 2-one }^{\text {b }}\end{array}$ & chiral \\
\hline 105 & 4 & & $\begin{array}{l}1,2,3,7,8,10- \\
\left.\text { hexachloropentacyclo[5.3.0.0 } 0^{2,6} \cdot 0^{3,9} \cdot 0^{4,8}\right] \text { decan-5-one }\end{array}$ & $\begin{array}{l}\text { 1,3a,4,5,5b,6-hexachlorooctahydro- } \\
\text { 1,3,4-metheno-2H- } \\
\text { cyclobuta[cd]pentalen-2-one }\end{array}$ & chiral \\
\hline 106 & 4 & & $\begin{array}{l}1,2,4,6,10,10- \\
\left.\text { hexachloropentacyclo[5.3.0.0 } 0^{2,6} \cdot 0^{3,9} \cdot 0^{4,8}\right] \text { decan-5-one }\end{array}$ & $\begin{array}{l}\text { 1,1a,3,5,5,5a-hexachlorooctahydro- } \\
\text { 1,3,4-metheno-2H- } \\
\text { cyclobuta[cd]pentalen-2-one }^{\mathbf{b}}\end{array}$ & chiral \\
\hline 107 & 4 & & $\begin{array}{l}1,2,6,9,10,10- \\
\left.\text { hexachloropentacyclo[5.3.0.0 } 0^{2,6} \cdot 0^{3,9} \cdot 0^{4,8}\right] \text { decan-5-one }\end{array}$ & $\begin{array}{l}\text { 1,1a,4,5,5,5a,5b- } \\
\text { hexachlorooctahydro-1,3,4- } \\
\text { metheno-2H-cyclobuta[cd]pentalen- } \\
\text { 2-one }^{\text {b }}\end{array}$ & chiral \\
\hline
\end{tabular}


Gibbs Free Energy of Formation of Chlordecone and Potential Degradation Products: Implications for Remediation Strategies and Environmental Fate Table SI-1 Structures of all chlordecone potential dechlorinated congeners and their IUPAC and CAS names

\begin{tabular}{|c|c|c|c|c|c|}
\hline Entry & $\overline{\mathrm{nCl}}$ & Structure $^{a}$ & IUPAC - Name & CAS - Name & Chirality \\
\hline 108 & 4 & & $\begin{array}{l}1,2,8,9,10,10- \\
\left.\text { hexachloropentacyclo[5.3.0.0 } 0^{2,6} \cdot 0^{3,9} \cdot 0^{4,8}\right] \text { decan-5-one }\end{array}$ & $\begin{array}{l}\text { 1,3a,4,5,5,5a-hexachlorooctahydro- } \\
\text { 1,3,4-metheno-2H- } \\
\text { cyclobuta[cd]pentalen-2-one }^{\text {b }}\end{array}$ & chiral \\
\hline 109 & 4 & & $\begin{array}{l}1,2,4,8,10,10- \\
\left.\text { hexachloropentacyclo[5.3.0.0 } 0^{2,6} \cdot 0^{3,9} \cdot 0^{4,8}\right] \text { decan-5-one }\end{array}$ & $\begin{array}{l}\text { 1,3,3a,5,5,5a-hexachlorooctahydro- } \\
\text { 1,3,4-metheno-2H- } \\
\text { cyclobuta[cd]pentalen-2-one }^{\text {b }}\end{array}$ & chiral \\
\hline 110 & 4 & & $\begin{array}{l}1,2,3,4,10,10- \\
\left.\text { hexachloropentacyclo[5.3.0.0 } 0^{2,6} \cdot 0^{3,9} \cdot 0^{4,8}\right] \text { decan-5-one }\end{array}$ & $\begin{array}{l}\text { 1,1a,4,5,5,6-hexachlorooctahydro- } \\
\text { 1,3,4-metheno-2H- } \\
\text { cyclobuta[cd]pentalen-2-one }\end{array}$ & chiral \\
\hline 111 & 5 & & $\begin{array}{l}2,3,4,7,8-p e n t a c h l o r o p e n t a c y c l o\left[5 \cdot 3 \cdot 0 \cdot 0^{2,6} \cdot 0^{3,9} \cdot 0^{4,8}\right] \\
\text { decan-5-one }\end{array}$ & $\begin{array}{l}\text { 1,1a,3a,5b,6-pentachlorooctahydro- } \\
\text { 1,3,4-metheno-2H- } \\
\text { cyclobuta[cd]pentalen-2-one }\end{array}$ & meso \\
\hline 112 & 5 & & $\begin{array}{l}\left.\text { 1,2,3,7,8-pentachloropentacyclo[5.3.0.0 } 0^{2,6} \cdot 0^{3,9} \cdot 0^{4,8}\right] \\
\text { decan-5-one }\end{array}$ & $\begin{array}{l}\text { 1,3a,4,5b,6-pentachlorooctahydro- } \\
\text { 1,3,4-metheno-2H- } \\
\text { cyclobuta[cd]pentalen-2-one }\end{array}$ & meso \\
\hline 113 & 5 & & $\begin{array}{l}\left.\text { 1,3,4,6,8-pentachloropentacyclo[5.3.0.0 } 0^{2,6} \cdot 0^{3,9} \cdot 0^{4,8}\right] \\
\text { decan-5-one }\end{array}$ & $\begin{array}{l}\text { 1,1a,3,4,5b-pentachlorooctahydro- } \\
\text { 1,3,4-metheno-2H- } \\
\text { cyclobuta[cd]pentalen-2-one }\end{array}$ & meso \\
\hline
\end{tabular}


Gibbs Free Energy of Formation of Chlordecone and Potential Degradation Products: Implications for Remediation Strategies and Environmental Fate Table SI-1 Structures of all chlordecone potential dechlorinated congeners and their IUPAC and CAS names

\begin{tabular}{|c|c|c|c|c|c|}
\hline Entry & $\overline{\mathrm{nCl}}$ & Structure $^{a}$ & IUPAC - Name & CAS - Name & Chirality \\
\hline 114 & 5 & H & $\begin{array}{l}\text { 1,2,4,7,9-pentachloropentacyclo }\left[5 \cdot 3 \cdot 0 \cdot 0^{2,6} \cdot 0^{3,9} \cdot 0^{4,8}\right] \\
\text { decan-5-one }\end{array}$ & $\begin{array}{l}\text { 1,3,4,5a,5b-pentachlorooctahydro- } \\
\text { 1,3,4-metheno-2H- } \\
\text { cyclobuta[cd]pentalen-2-one }^{\mathbf{b}}\end{array}$ & meso \\
\hline 115 & 5 & $\mathbf{H}$ & $\begin{array}{l}\left.\text { 1,2,6,7,9-pentachloropentacyclo[5.3.0.0 } 0^{2,6} \cdot 0^{3,9} \cdot 0^{4,8}\right] \\
\text { decan-5-one }\end{array}$ & $\begin{array}{l}\text { 1,1a,4,5a,5b-pentachlorooctahydro- } \\
\text { 1,3,4-metheno-2H- } \\
\text { cyclobuta[cd]pentalen-2-one }^{\mathbf{b}}\end{array}$ & meso \\
\hline 116 & 5 & & $\begin{array}{l}\left.\text { 1,2,4,6,7-pentachloropentacyclo[5.3.0.0 } 2,6 \cdot 0^{3,9} \cdot 0^{4,8}\right] \\
\text { decan-5-one }\end{array}$ & $\begin{array}{l}\text { 1,1a,3,5a,5b-pentachlorooctahydro- } \\
\text { 1,3,4-metheno-2H- } \\
\text { cyclobuta[cd]pentalen-2-one }^{\text {b }}\end{array}$ & meso \\
\hline 117 & 5 & & $\begin{array}{l}2,6,7,10,10-\text { pentachloropentacyclo }\left[5 \cdot 3 \cdot 0 \cdot 0^{2,6} \cdot 0^{3,9} \cdot 0^{4,8}\right] \\
\text { decan-5-one }\end{array}$ & $\begin{array}{l}\text { 1,1a,5,5,5b-pentachlorooctahydro- } \\
\text { 1,3,4-metheno-2H- } \\
\text { cyclobuta[cd]pentalen-2-one }^{\mathbf{b}}\end{array}$ & meso \\
\hline 118 & 5 & & $\begin{array}{l}2,4,7,10,10-\text { pentachloropentacyclo }\left[5 \cdot 3 \cdot 0 \cdot 0^{2,6} \cdot 0^{3,9} \cdot 0^{4,8}\right] \\
\text { decan-5-one }\end{array}$ & $\begin{array}{l}\text { 1,3,5,5,5b-pentachlorooctahydro- } \\
\text { 1,3,4-metheno-2H- } \\
\text { cyclobuta[cd]pentalen-2-one }^{\mathbf{b}}\end{array}$ & meso \\
\hline 119 & 5 & & $\begin{array}{l}\text { 1,2,7,10,10-pentachloropentacyclo }\left[5 \cdot 3 \cdot 0 \cdot 0^{2,6} \cdot 0^{3,9} \cdot 0^{4,8}\right] \\
\text { decan-5-one }\end{array}$ & $\begin{array}{l}\text { 1,5,5,5a,5b-pentachlorooctahydro- } \\
\text { 1,3,4-metheno-2H- } \\
\text { cyclobuta[cd]pentalen-2-one }^{\mathbf{b}}\end{array}$ & meso \\
\hline
\end{tabular}


Gibbs Free Energy of Formation of Chlordecone and Potential Degradation Products: Implications for Remediation Strategies and Environmental Fate Table SI-1 Structures of all chlordecone potential dechlorinated congeners and their IUPAC and CAS names

\begin{tabular}{|c|c|c|c|c|c|}
\hline Entry & $\overline{\mathrm{nCl}}$ & Structure $^{a}$ & IUPAC - Name & CAS - Name & Chirality \\
\hline 120 & 5 & $\mathrm{H}$ & $\begin{array}{l}1,3,8,10,10- \\
\left.\text { pentachloropentacyclo[5.3.0.0 } 0^{2,6} \cdot 0^{3,9} \cdot 0^{4,8}\right] \text { decan-5-one }\end{array}$ & $\begin{array}{l}\text { 1,4,5,5,5b-pentachlorooctahydro- } \\
\text { 1,3,4-metheno-2H- } \\
\text { cyclobuta[cd]pentalen-2-one }\end{array}$ & meso \\
\hline 121 & 5 & & $\begin{array}{l}1, r-2,3,9, c-10- \\
\text { pentachloropentacyclo }\left[5 \cdot 3 \cdot 0 \cdot 0^{2,6} \cdot 0^{3,9} \cdot 0^{4,8}\right] \text { decan-5-one }\end{array}$ & $\begin{array}{l}r \text {-1,4,c-5,5a,6-pentachlorooctahydro- } \\
\text { 1,3,4-metheno-2H- } \\
\text { cyclobuta[cd]pentalen-2-one }\end{array}$ & meso \\
\hline 122 & 5 & & $\begin{array}{l}1, r-2,3,9, t-10- \\
\text { pentachloropentacyclo }\left[5 \cdot 3 \cdot 0 \cdot 0^{2,6} \cdot 0^{3,9} \cdot 0^{4,8}\right] \text { decan-5-one }\end{array}$ & $\begin{array}{l}r \text {-1,4,t-5,5a,6-pentachlorooctahydro- } \\
\text { 1,3,4-metheno-2H- } \\
\text { cyclobuta[cd]pentalen-2-one }\end{array}$ & meso \\
\hline 123 & 5 & & $\begin{array}{l}1,4,9,10,10- \\
\text { pentachloropentacyclo }\left[5 \cdot 3 \cdot 0 \cdot 0^{2,6} \cdot 0^{3,9} \cdot 0^{4,8}\right] \text { decan-5-one }\end{array}$ & $\begin{array}{l}\text { 1a,4,5,5,5a-pentachlorooctahydro- } \\
\text { 1,3,4-metheno-2H- } \\
\text { cyclobuta[cd]pentalen-2-one }\end{array}$ & meso \\
\hline 124 & 5 & & $\begin{array}{l}r-2,3,4,6, t-10- \\
\text { pentachloropentacyclo }\left[5 \cdot 3 \cdot 0 \cdot 0^{2,6} \cdot 0^{3,9} \cdot 0^{4,8}\right] \text { decan-5-one }\end{array}$ & $\begin{array}{l}\text { r-1,1a,3,t-5,6-pentachlorooctahydro- } \\
\text { 1,3,4-metheno-2H- } \\
\text { cyclobuta[cd]pentalen-2-one }\end{array}$ & meso \\
\hline 125 & 5 & & $\begin{array}{l}r-2,3,4,6, c-10- \\
\text { pentachloropentacyclo }\left[5 \cdot 3 \cdot 0 \cdot 0^{2,6} \cdot 0^{3,9} \cdot 0^{4,8}\right] \text { decan-5-one }\end{array}$ & $\begin{array}{l}\text { r-1,1a,3,c-5,6-pentachlorooctahydro- } \\
\text { 1,3,4-metheno-2H- } \\
\text { cyclobuta[cd]pentalen-2-one }\end{array}$ & meso \\
\hline 126 & 5 & & $\begin{array}{l}1,4,6,9,10- \\
\text { pentachloropentacyclo }\left[5 \cdot 3 \cdot 0 \cdot 0^{2,6} \cdot 0^{3,9} \cdot 0^{4,8}\right] \text { decan-5-one }\end{array}$ & $\begin{array}{l}\text { 1a,3,4,5,5a-pentachlorooctahydro- } \\
\text { 1,3,4-metheno-2H- } \\
\text { cyclobuta[cd]pentalen-2-one }\end{array}$ & meso \\
\hline
\end{tabular}


Gibbs Free Energy of Formation of Chlordecone and Potential Degradation Products: Implications for Remediation Strategies and Environmental Fate Table SI-1 Structures of all chlordecone potential dechlorinated congeners and their IUPAC and CAS names

\begin{tabular}{|c|c|c|c|c|c|}
\hline Entry & $\overline{\mathrm{nCl}}$ & Structure $^{a}$ & IUPAC - Name & CAS - Name & Chirality \\
\hline 127 & 5 & $\mathbf{H}$ & $\begin{array}{l}2,3,7,8,10- \\
\left.\text { pentachloropentacyclo[5.3.0.0 } 0^{2,6} \cdot 0^{3,9} \cdot 0^{4,8}\right] \text { decan-5-one }\end{array}$ & $\begin{array}{l}\text { 1,3a,5,5b,6-pentachlorooctahydro- } \\
\text { 1,3,4-metheno-2H- } \\
\text { cyclobuta[cd]pentalen-2-one }\end{array}$ & meso \\
\hline 128 & 5 & & $\begin{array}{l}1,4,6,10,10- \\
\left.\text { pentachloropentacyclo[5.3.0.0 } 0^{2,6} \cdot 0^{3,9} \cdot 0^{4,8}\right] \text { decan-5-one }\end{array}$ & $\begin{array}{l}\text { 1a,3,4,5,5-pentachlorooctahydro- } \\
\text { 1,3,4-metheno-2H- } \\
\text { cyclobuta[cd]pentalen-2-one }\end{array}$ & meso \\
\hline 129 & 5 & & $\begin{array}{l}2,3,4,6,7- \\
\left.\text { pentachloropentacyclo[5.3.0.0 } 0^{2,6} \cdot 0^{3,9} \cdot 0^{4,8}\right] \text { decan-5-one }\end{array}$ & $\begin{array}{l}\text { 1,1a,3,3a,5b-pentachlorooctahydro- } \\
\text { 1,3,4-metheno- } 2 \mathrm{H}- \\
\text { cyclobuta[cd]pentalen-2-one }^{c}\end{array}$ & chiral \\
\hline 130 & 5 & & $\begin{array}{l}1,3,4,6,7- \\
\left.\text { pentachloropentacyclo[5.3.0.0 } 0^{2,6} \cdot 0^{3,9} \cdot 0^{4,8}\right] \text { decan-5-one }\end{array}$ & $\begin{array}{l}\text { 1,1a,3,3a,4-pentachlorooctahydro- } \\
\text { 1,3,4-metheno-2H- } \\
\text { cyclobuta[cd]pentalen-2-one }\end{array}$ & chiral \\
\hline 131 & 5 & & $\begin{array}{l}1,2,3,4,7- \\
\left.\text { pentachloropentacyclo[5.3.0.0 } 0^{2,6} \cdot 0^{3,9} \cdot 0^{4,8}\right] \text { decan-5-one }\end{array}$ & $\begin{array}{l}\text { 1,1a,3a,4,6-pentachlorooctahydro- } \\
\text { 1,3,4-metheno-2H- } \\
\text { cyclobuta[cd]pentalen-2-one }\end{array}$ & chiral \\
\hline 132 & 5 & & $\begin{array}{l}1,2,3,4,6- \\
\text { pentachloropentacyclo }\left[5 \cdot 3 \cdot 0 \cdot 0^{2,6} \cdot 0^{3,9} \cdot 0^{4,8}\right] \text { decan-5-one }\end{array}$ & $\begin{array}{l}\text { 1,1a,3,4,6-pentachlorooctahydro- } \\
\text { 1,3,4-metheno-2H- } \\
\text { cyclobuta[cd]pentalen-2-one }\end{array}$ & chiral \\
\hline
\end{tabular}


Gibbs Free Energy of Formation of Chlordecone and Potential Degradation Products: Implications for Remediation Strategies and Environmental Fate Table SI-1 Structures of all chlordecone potential dechlorinated congeners and their IUPAC and CAS names

\begin{tabular}{|c|c|c|c|c|c|}
\hline Entry & $\overline{\mathrm{nCl}}$ & Structure $^{a}$ & IUPAC - Name & CAS - Name & Chirality \\
\hline 133 & 5 & $\mathbf{H}$ & $\begin{array}{l}1,3,6,7,8- \\
\left.\text { pentachloropentacyclo[5.3.0.0 } 0^{2,6} \cdot 0^{3,9} \cdot 0^{4,8}\right] \text { decan-5-one }\end{array}$ & $\begin{array}{l}\text { 1,1a,3a,5a,6-pentachlorooctahydro- } \\
\text { 1,3,4-metheno- } 2 \mathrm{H}- \\
\text { cyclobuta[cd]pentalen-2-one }^{c}\end{array}$ & chiral \\
\hline 134 & 5 & & $\begin{array}{l}1,2,4,6,9- \\
\left.\text { pentachloropentacyclo[5.3.0.0 } 0^{2,6} \cdot 0^{3,9} \cdot 0^{4,8}\right] \text { decan-5-one }\end{array}$ & $\begin{array}{l}\text { 1,1a,3,4,5a-pentachlorooctahydro- } \\
\text { 1,3,4-metheno-2H- } \\
\text { cyclobuta[cd]pentalen-2-one }^{\text {b }}\end{array}$ & chiral \\
\hline 135 & 5 & & $\begin{array}{l}2,4,6,7,10- \\
\left.\text { pentachloropentacyclo[5.3.0.0 } 0^{2,6} \cdot 0^{3,9} \cdot 0^{4,8}\right] \text { decan-5-one }\end{array}$ & $\begin{array}{l}\text { 1,1a,3,5,5b-pentachlorooctahydro- } \\
\text { 1,3,4-metheno- } 2 \mathrm{H} \text { - } \\
\text { cyclobuta[cd]pentalen-2-one }^{\mathrm{b}}\end{array}$ & chiral \\
\hline 136 & 5 & & $\begin{array}{l}1, r-2,4,6, t-10- \\
\left.\text { pentachloropentacyclo[5.3.0.0 } 0^{2,6} \cdot 0^{3,9} \cdot 0^{4,8}\right] \text { decan-5-one }\end{array}$ & $\begin{array}{l}r-1,1 \mathrm{a}, 3, t-5,5 \mathrm{a}- \\
\text { pentachlorooctahydro-1,3,4- } \\
\text { metheno-2H-cyclobuta[cd]pentalen- } \\
\text { 2-one }^{b}\end{array}$ & chiral \\
\hline 137 & 5 & & $\begin{array}{l}1, r-2,4,6, c-10- \\
\left.\text { pentachloropentacyclo[5.3.0.0 } 0^{2,6} \cdot 0^{3,9} \cdot 0^{4,8}\right] \text { decan-5-one }\end{array}$ & $\begin{array}{l}r-1,1 \mathrm{a}, 3, c-5,5 \mathrm{a}- \\
\text { pentachlorooctahydro-1,3,4- } \\
\text { metheno-2H-cyclobuta[cd]pentalen- } \\
\text { 2-one }{ }^{b}\end{array}$ & chiral \\
\hline 138 & 5 & & $\begin{array}{l}1,2,4,7,10- \\
\left.\text { pentachloropentacyclo[5.3.0.0 } 0^{2,6} \cdot 0^{3,9} \cdot 0^{4,8}\right] \text { decan-5-one }\end{array}$ & $\begin{array}{l}\text { 1,3,5,5a,5b-pentachlorooctahydro- } \\
\text { 1,3,4-metheno-2H- } \\
\text { cyclobuta[cd]pentalen-2-one }^{\text {b }}\end{array}$ & chiral \\
\hline
\end{tabular}


Gibbs Free Energy of Formation of Chlordecone and Potential Degradation Products: Implications for Remediation Strategies and Environmental Fate Table SI-1 Structures of all chlordecone potential dechlorinated congeners and their IUPAC and CAS names

\begin{tabular}{|c|c|c|c|c|c|}
\hline Entry & $\overline{\mathrm{nCl}}$ & Structure $^{a}$ & IUPAC - Name & CAS - Name & Chirality \\
\hline 139 & 5 & $\overrightarrow{\mathbf{H}}$ & $\begin{array}{l}\mathrm{r}-2,3,4,8, c-10- \\
\text { pentachloropentacyclo }\left[5 \cdot 3 \cdot 0 \cdot 0^{2,6} \cdot 0^{3,9} \cdot 0^{4,8}\right] \text { decan-5-one }\end{array}$ & $\begin{array}{l}\mathrm{r}-1,1 \mathrm{a}, \mathrm{c}-5,5 \mathrm{~b}, 6- \\
\text { pentachlorooctahydro-1,3,4- } \\
\text { metheno-2H-cyclobuta[cd]pentalen- } \\
\text { 2-one }\end{array}$ & chiral \\
\hline 140 & 5 & & $\begin{array}{l}\mathrm{r}-2,3,4,8, t-10- \\
\left.\text { pentachloropentacyclo[5.3.0.0 } 0^{2,6} \cdot 0^{3,9} \cdot 0^{4,8}\right] \text { decan-5-one }\end{array}$ & $\begin{array}{l}\mathrm{r}-1,1 \mathrm{a}, t-5,5 \mathrm{~b}, 6- \\
\text { pentachlorooctahydro-1,3,4- } \\
\text { metheno-2H-cyclobuta[cd]pentalen- } \\
\text { 2-one }\end{array}$ & chiral \\
\hline 141 & 5 & & $\begin{array}{l}1, r-3,6,7, c-10- \\
\left.\text { pentachloropentacyclo[5.3.0.0 } 0^{2,6} \cdot 0^{3,9} \cdot 0^{4,8}\right] \text { decan-5-one }\end{array}$ & $\begin{array}{l}r-1,1 \mathrm{a}, 3 \mathrm{a}, c-5,5 \mathrm{a}- \\
\text { pentachlorooctahydro-1,3,4- } \\
\text { metheno-2H-cyclobuta[cd]pentalen- } \\
\text { 2-one }{ }^{c}\end{array}$ & chiral \\
\hline 142 & 5 & & $\begin{array}{l}1, r-3,6,7, t-10- \\
\left.\text { pentachloropentacyclo[5.3.0.0 } 0^{2,6} \cdot 0^{3,9} \cdot 0^{4,8}\right] \text { decan-5-one }\end{array}$ & $\begin{array}{l}r-1,1 \mathrm{a}, 3 \mathrm{a}, t-5,5 \mathrm{a}- \\
\text { pentachlorooctahydro-1,3,4- } \\
\text { metheno-2H-cyclobuta[cd]pentalen- } \\
\text { 2-one }{ }^{c}\end{array}$ & chiral \\
\hline 143 & 5 & & $\begin{array}{l}1, r-2,3,7, c-10- \\
\left.\text { pentachloropentacyclo[5.3.0.0 } 0^{2,6} \cdot 0^{3,9} \cdot 0^{4,8}\right] \text { decan-5-one }\end{array}$ & $\begin{array}{l}r \text {-1,3a,4,c-5,6-pentachlorooctahydro- } \\
\text { 1,3,4-metheno-2H- } \\
\text { cyclobuta[cd]pentalen-2-one }\end{array}$ & chiral \\
\hline 144 & 5 & & $\begin{array}{l}1, r-2,3,7, t-10- \\
\text { pentachloropentacyclo }\left[5 \cdot 3 \cdot 0 \cdot 0^{2,6} \cdot 0^{3,9} \cdot 0^{4,8}\right] \text { decan-5-one }\end{array}$ & $\begin{array}{l}r \text {-1,3a,4,t-5,6-pentachlorooctahydro- } \\
\text { 1,3,4-metheno-2H- } \\
\text { cyclobuta[cd]pentalen-2-one }\end{array}$ & chiral \\
\hline
\end{tabular}


Gibbs Free Energy of Formation of Chlordecone and Potential Degradation Products: Implications for Remediation Strategies and Environmental Fate Table SI-1 Structures of all chlordecone potential dechlorinated congeners and their IUPAC and CAS names

\begin{tabular}{|c|c|c|c|c|c|}
\hline Entry & $\overline{\mathrm{nCl}}$ & Structure $^{a}$ & IUPAC - Name & CAS - Name & Chirality \\
\hline 145 & 5 & & $\begin{array}{l}1, r-2,3,6, c-10- \\
\left.\text { pentachloropentacyclo[5.3.0.0 } 0^{2,6} \cdot 0^{3,9} \cdot 0^{4,8}\right] \text { decan-5-one }\end{array}$ & $\begin{array}{l}r-1,1 \mathrm{a}, c-5,5 \mathrm{a}, 6- \\
\text { pentachlorooctahydro-1,3,4- } \\
\text { metheno-2H-cyclobuta[cd]pentalen- } \\
\text { 2-one } \mathrm{b}^{\mathrm{b}}\end{array}$ & chiral \\
\hline 146 & 5 & & $\begin{array}{l}1, r-2,3,6, t-10- \\
\left.\text { pentachloropentacyclo[5.3.0.0 } 0^{2,6} \cdot 0^{3,9} \cdot 0^{4,8}\right] \text { decan-5-one }\end{array}$ & $\begin{array}{l}r-1,1 \mathrm{a}, t-5,5 \mathrm{a}, 6- \\
\text { pentachlorooctahydro-1,3,4- } \\
\text { metheno-2H-cyclobuta[cd]pentalen- } \\
\text { 2-one }{ }^{\mathrm{b}}\end{array}$ & chiral \\
\hline 147 & 5 & & $\begin{array}{l}1,2,6,7,10- \\
\left.\text { pentachloropentacyclo[5.3.0.0 } 0^{2,6} \cdot 0^{3,9} \cdot 0^{4,8}\right] \text { decan-5-one }\end{array}$ & $\begin{array}{l}\text { 1,1a,5,5a,5b-pentachlorooctahydro- } \\
\text { 1,3,4-metheno-2H- } \\
\text { cyclobuta[cd]pentalen-2-one }^{b}\end{array}$ & chiral \\
\hline 148 & 5 & & $\begin{array}{l}1,3,4,8,10 \text { - } \\
\left.\text { pentachloropentacyclo[5.3.0.0 } 0^{2,6} \cdot 0^{3,9} \cdot 0^{4,8}\right] \text { decan-5-one }\end{array}$ & $\begin{array}{l}\text { 1,1a,4,5,5b-pentachlorooctahydro- } \\
\text { 1,3,4-metheno-2H- } \\
\text { cyclobuta[cd]pentalen-2-one }\end{array}$ & chiral \\
\hline 149 & 5 & & $\begin{array}{l}1, r-2,6,9, t-10- \\
\left.\text { pentachloropentacyclo[5.3.0.0 } 0^{2,6} \cdot 0^{3,9} \cdot 0^{4,8}\right] \text { decan-5-one }\end{array}$ & $\begin{array}{l}r-1,1 \mathrm{a}, 4, t-5,5 \mathrm{a}- \\
\text { pentachlorooctahydro-1,3,4- } \\
\text { metheno-2H-cyclobuta[cd]pentalen- } \\
\text { 2-one }{ }^{\mathrm{b}}\end{array}$ & chiral \\
\hline 150 & 5 & & $\begin{array}{l}1, r-2,6,9, c-10- \\
\left.\text { pentachloropentacyclo[5.3.0.0 } 0^{2,6} \cdot 0^{3,9} \cdot 0^{4,8}\right] \text { decan-5-one }\end{array}$ & $\begin{array}{l}r-1,1 \mathrm{a}, 4, c-5,5 \mathrm{a}- \\
\text { pentachlorooctahydro-1,3,4- } \\
\text { metheno-2H-cyclobuta[cd]pentalen- } \\
\text { 2-one }{ }^{\mathrm{b}}\end{array}$ & chiral \\
\hline 151 & 5 & & $\begin{array}{l}1,2,7,9,10- \\
\left.\text { pentachloropentacyclo[5.3.0.0 } 0^{2,6} \cdot 0^{3,9} \cdot 0^{4,8}\right] \text { decan-5-one }\end{array}$ & $\begin{array}{l}\text { 1,4,5,5a,5b-pentachlorooctahydro- } \\
\text { 1,3,4-metheno- } 2 \mathrm{H} \text { - } \\
\text { cyclobuta[cd]pentalen-2-one }^{\mathrm{b}}\end{array}$ & chiral \\
\hline
\end{tabular}


Gibbs Free Energy of Formation of Chlordecone and Potential Degradation Products: Implications for Remediation Strategies and Environmental Fate Table SI-1 Structures of all chlordecone potential dechlorinated congeners and their IUPAC and CAS names

\begin{tabular}{|c|c|c|c|c|c|}
\hline Entry & $\overline{\mathrm{nCl}}$ & Structure $^{a}$ & IUPAC - Name & CAS - Name & Chirality \\
\hline 152 & 5 & & $\begin{array}{l}1,6,7,10,10- \\
\text { pentachloropentacyclo }\left[5 \cdot 3 \cdot 0 \cdot 0^{2,6} \cdot 0^{3,9} \cdot 0^{4,8}\right] \text { decan-5-one }\end{array}$ & $\begin{array}{l}\text { 1,1a,5,5,5a-pentachlorooctahydro- } \\
\text { 1,3,4-metheno-2H- } \\
\text { cyclobuta[cd]pentalen-2-one }^{c}\end{array}$ & chiral \\
\hline 153 & 5 & & $\begin{array}{l}2,3,6,10,10- \\
\left.\text { pentachloropentacyclo[5.3.0.0 } 0^{2,6} \cdot 0^{3,9} \cdot 0^{4,8}\right] \text { decan-5-one }\end{array}$ & $\begin{array}{l}\text { 1,1a,5,5,6-pentachlorooctahydro- } \\
\text { 1,3,4-metheno- } 2 \mathrm{H} \text { - } \\
\text { cyclobuta[cd]pentalen-2-one }^{\mathbf{b}}\end{array}$ & chiral \\
\hline 154 & 5 & & $\begin{array}{l}2,3,7,10,10- \\
\text { pentachloropentacyclo }\left[5 \cdot 3 \cdot 0 \cdot 0^{2,6} \cdot 0^{3,9} \cdot 0^{4,8}\right] \text { decan-5-one }\end{array}$ & $\begin{array}{l}\text { 1,3a,5,5,5b-pentachlorooctahydro- } \\
\text { 1,3,4-metheno-2H- } \\
\text { cyclobuta[cd]pentalen-2-one }^{c}\end{array}$ & chiral \\
\hline 155 & 5 & & $\begin{array}{l}2,4,8,10,10- \\
\text { pentachloropentacyclo }\left[5 \cdot 3 \cdot 0 \cdot 0^{2,6} \cdot 0^{3,9} \cdot 0^{4,8}\right] \text { decan-5-one }\end{array}$ & $\begin{array}{l}\text { 1,3,3a,5,5-pentachlorooctahydro- } \\
\text { 1,3,4-metheno- } 2 \mathrm{H} \text { - } \\
\text { cyclobuta[cd]pentalen-2-one }^{\mathbf{b}}\end{array}$ & chiral \\
\hline 156 & 5 & & $\begin{array}{l}1,2,3,10,10- \\
\text { pentachloropentacyclo }\left[5 \cdot 3 \cdot 0 \cdot 0^{2,6} \cdot 0^{3,9} \cdot 0^{4,8}\right] \text { decan-5-one }\end{array}$ & $\begin{array}{l}\text { 1,4,5,5,6-pentachlorooctahydro- } \\
\text { 1,3,4-metheno-2H- } \\
\text { cyclobuta[cd]pentalen-2-one }\end{array}$ & chiral \\
\hline 157 & 5 & & $\begin{array}{l}\text { 1,3,6,10,10- } \\
\left.\text { pentachloropentacyclo[5.3.0.0 } 0^{2,6} \cdot 0^{3,9} \cdot 0^{4,8}\right] \text { decan-5-one }\end{array}$ & $\begin{array}{l}\text { 1,3,4,5,5-pentachlorooctahydro- } \\
\text { 1,3,4-metheno-2H- } \\
\text { cyclobuta[cd]pentalen-2-one }\end{array}$ & chiral \\
\hline 158 & 5 & & $\begin{array}{l}1,2,8,10,10- \\
\left.\text { pentachloropentacyclo[5.3.0.0 } 0^{2,6} \cdot 0^{3,9} \cdot 0^{4,8}\right] \text { decan-5-one }\end{array}$ & $\begin{array}{l}\text { 1,3a,5,5,5a-pentachlorooctahydro- } \\
\text { 1,3,4-metheno-2H- } \\
\text { cyclobuta[cd]pentalen-2-one }^{\text {b }}\end{array}$ & chiral \\
\hline
\end{tabular}


Gibbs Free Energy of Formation of Chlordecone and Potential Degradation Products: Implications for Remediation Strategies and Environmental Fate Table SI-1 Structures of all chlordecone potential dechlorinated congeners and their IUPAC and CAS names

\begin{tabular}{|c|c|c|c|c|c|}
\hline Entry & $\overline{\mathrm{nCl}}$ & Structure $^{a}$ & IUPAC - Name & CAS - Name & Chirality \\
\hline 159 & 5 & & $\begin{array}{l}1,3,4,10,10- \\
\left.\text { pentachloropentacyclo[5.3.0.0 } 0^{2,6} \cdot 0^{3,9} \cdot 0^{4,8}\right] \text { decan-5-one }\end{array}$ & $\begin{array}{l}\text { 1,1a,4,5,5-pentachlorooctahydro- } \\
\text { 1,3,4-metheno-2H- } \\
\text { cyclobuta[cd]pentalen-2-one }\end{array}$ & chiral \\
\hline 160 & 5 & & $\begin{array}{l}1,2,9,10,10- \\
\text { pentachloropentacyclo }\left[5 \cdot 3 \cdot 0 \cdot 0^{2,6} \cdot 0^{3,9} \cdot 0^{4,8}\right] \text { decan-5-one }\end{array}$ & $\begin{array}{l}\text { 1,4,5,5,5a-pentachlorooctahydro- } \\
\text { 1,3,4-metheno-2H- } \\
\text { cyclobuta[cd]pentalen-2-one }^{\text {b }}\end{array}$ & chiral \\
\hline 161 & 5 & & $\begin{array}{l}r-2,3,6,8, t-10- \\
\text { pentachloropentacyclo[5.3.0.0. } \\
\left.2,6 \cdot 0^{3,9} \cdot 0^{4,8}\right] \text { decan-5-one }\end{array}$ & $\begin{array}{l}r-1,1 \mathrm{a}, 3 \mathrm{a}, t-5,6- \\
\text { pentachlorooctahydro-1,3,4- } \\
\text { metheno-2H-cyclobuta[cd]pentalen- } \\
\text { 2-one }{ }^{b}\end{array}$ & chiral \\
\hline 162 & 5 & & $\begin{array}{l}r-2,3,6,8, c-10- \\
\left.\text { pentachloropentacyclo[5.3.0.0 } 0^{2,6} \cdot 0^{3,9} \cdot 0^{4,8}\right] \text { decan-5-one }\end{array}$ & $\begin{array}{l}r-1,1 \mathrm{a}, 3 \mathrm{a}, c-5,6- \\
\text { pentachlorooctahydro-1,3,4- } \\
\text { metheno-2H-cyclobuta[cd]pentalen- } \\
\text { 2-one }{ }^{b}\end{array}$ & chiral \\
\hline 163 & 5 & & $\begin{array}{l}2,4,6,8,10 \text { - } \\
\left.\text { pentachloropentacyclo[5.3.0.0 } 0^{2,6} \cdot 0^{3,9} \cdot 0^{4,8}\right] \text { decan-5-one }\end{array}$ & $\begin{array}{l}\text { 1,1a,3,3a,5-pentachlorooctahydro- } \\
\text { 1,3,4-metheno-2H- } \\
\text { cyclobuta[cd]pentalen-2-one }^{b}\end{array}$ & chiral \\
\hline 164 & 5 & & $\begin{array}{l}1, r-2,3,4, t-10- \\
\left.\text { pentachloropentacyclo[5.3.0.0 } 0^{2,6} \cdot 0^{3,9} \cdot 0^{4,8}\right] \text { decan-5-one }\end{array}$ & $\begin{array}{l}r \text {-1,1a,4,t-5,6-pentachlorooctahydro- } \\
\text { 1,3,4-metheno-2H- } \\
\text { cyclobuta[cd]pentalen-2-one }\end{array}$ & chiral \\
\hline 165 & 5 & & $\begin{array}{l}1, r-2,3,4, c-10- \\
\text { pentachloropentacyclo }\left[5 \cdot 3 \cdot 0 \cdot 0^{2,6} \cdot 0^{3,9} \cdot 0^{4,8}\right] \text { decan-5-one }\end{array}$ & $\begin{array}{l}r \text {-1,1a,4,c-5,6-pentachlorooctahydro- } \\
\text { 1,3,4-metheno-2H- } \\
\text { cyclobuta[cd]pentalen-2-one }\end{array}$ & chiral \\
\hline
\end{tabular}


Gibbs Free Energy of Formation of Chlordecone and Potential Degradation Products: Implications for Remediation Strategies and Environmental Fate Table SI-1 Structures of all chlordecone potential dechlorinated congeners and their IUPAC and CAS names

\begin{tabular}{|c|c|c|c|c|c|}
\hline Entry & $\overline{\mathrm{nCl}}$ & Structure $^{a}$ & IUPAC - Name & CAS - Name & Chirality \\
\hline 166 & 5 & & $\begin{array}{l}1, r-2,4,8, t-10- \\
\text { pentachloropentacyclo }\left[5 \cdot 3 \cdot 0 \cdot 0^{2,6} \cdot 0^{3,9} \cdot 0^{4,8}\right] \text { decan-5-one }\end{array}$ & $\begin{array}{l}r-1,3,3 a, t-5,5 a- \\
\text { pentachlorooctahydro-1,3,4- } \\
\text { metheno-2H-cyclobuta[cd]pentalen- } \\
\text { 2-one }{ }^{b}\end{array}$ & chiral \\
\hline 167 & 5 & H & $\begin{array}{l}1, r-2,4,8, c-10- \\
\text { pentachloropentacyclo }\left[5 \cdot 3 \cdot 0 \cdot 0^{2,6} \cdot 0^{3,9} \cdot 0^{4,8}\right] \text { decan-5-one }\end{array}$ & $\begin{array}{l}r-1,3,3 a, c-5,5 a- \\
\text { pentachlorooctahydro-1,3,4- } \\
\text { metheno-2H-cyclobuta[cd]pentalen- } \\
\text { 2-one }{ }^{b}\end{array}$ & chiral \\
\hline 168 & 5 & & $\begin{array}{l}1, r-2,3,8, c-10- \\
\left.\text { pentachloropentacyclo[5.3.0.0 } 0^{2,6} \cdot 0^{3,9} \cdot 0^{4,8}\right] \text { decan-5-one }\end{array}$ & $\begin{array}{l}\text { r-1,4,c-5,5b,6-pentachlorooctahydro- } \\
\text { 1,3,4-metheno-2H- } \\
\text { cyclobuta[cd]pentalen-2-one }\end{array}$ & chiral \\
\hline 169 & 5 & & $\begin{array}{l}1, r-2,3,8, t-10- \\
\left.\text { pentachloropentacyclo[5.3.0.0 } 0^{2,6} \cdot 0^{3,9} \cdot 0^{4,8}\right] \text { decan-5-one }\end{array}$ & $\begin{array}{l}r-1,4, t-5,5 b, 6-p e n t a c h l o r o o c t a h y d r o- \\
\text { 1,3,4-metheno-2H- } \\
\text { cyclobuta[cd]pentalen-2-one }\end{array}$ & chiral \\
\hline 170 & 5 & & $\begin{array}{l}1, r-3,4,6, t-10- \\
\left.\text { pentachloropentacyclo[5.3.0.0 } 0^{2,6} \cdot 0^{3,9} \cdot 0^{4,8}\right] \text { decan-5-one }\end{array}$ & $\begin{array}{l}r-1,1 \mathrm{a}, 3,4, t-5-\text { pentachlorooctahydro- } \\
\text { 1,3,4-metheno-2H- } \\
\text { cyclobuta[cd]pentalen-2-one }\end{array}$ & chiral \\
\hline 171 & 5 & & $\begin{array}{l}1, r-3,4,6, c-10- \\
\left.\text { pentachloropentacyclo[5.3.0.0 } 0^{2,6} \cdot 0^{3,9} \cdot 0^{4,8}\right] \text { decan-5-one }\end{array}$ & $\begin{array}{l}r \text {-1,1a,3,4,c-5-pentachlorooctahydro- } \\
\text { 1,3,4-metheno-2H- } \\
\text { cyclobuta5[cd]pentalen-2-one }\end{array}$ & chiral \\
\hline 172 & 5 & & $\begin{array}{l}1, r-2,4,9, t-10- \\
\left.\text { pentachloropentacyclo[5.3.0.0 } 0^{2,6} \cdot 0^{3,9} \cdot 0^{4,8}\right] \text { decan-5-one }\end{array}$ & $\begin{array}{l}r-1,3,4, t-5,5 a-p e n t a c h l o r o o c t a h y d r o- \\
\text { 1,3,4-metheno-2H- } \\
\text { cyclobuta[cd]pentalen-2-one }^{b}\end{array}$ & chiral \\
\hline
\end{tabular}


Gibbs Free Energy of Formation of Chlordecone and Potential Degradation Products: Implications for Remediation Strategies and Environmental Fate Table SI-1 Structures of all chlordecone potential dechlorinated congeners and their IUPAC and CAS names

\begin{tabular}{|c|c|c|c|c|c|}
\hline Entry & $\overline{\mathrm{nCl}}$ & Structure $^{a}$ & IUPAC - Name & CAS - Name & Chirality \\
\hline 173 & 5 & & $\begin{array}{l}1, r-2,4,9, c-10- \\
\text { pentachloropentacyclo }\left[5 \cdot 3 \cdot 0 \cdot 0^{2,6} \cdot 0^{3,9} \cdot 0^{4,8}\right] \text { decan-5-one }\end{array}$ & $\begin{array}{l}r \text {-1,3,4,c-5,5a-pentachlorooctahydro- } \\
\text { 1,3,4-metheno-2H- } \\
\text { cyclobuta[cd]pentalen-2-one }^{b}\end{array}$ & chiral \\
\hline 174 & 5 & & $\begin{array}{l}1,3,6,8,10- \\
\text { pentachloropentacyclo }\left[5 \cdot 3 \cdot 0 \cdot 0^{2,6} \cdot 0^{3,9} \cdot 0^{4,8}\right] \text { decan-5-one }\end{array}$ & $\begin{array}{l}\text { 1,3,4,5,5b-pentachlorooctahydro- } \\
\text { 1,3,4-metheno-2H- } \\
\text { cyclobuta[cd]pentalen-2-one }\end{array}$ & chiral \\
\hline 175 & 5 & & $\begin{array}{l}1, r-2,6,8, c-10- \\
\text { pentachloropentacyclo }\left[5 \cdot 3 \cdot 0 \cdot 0^{2,6} \cdot 0^{3,9} \cdot 0^{4,8}\right] \text { decan-5-one }\end{array}$ & $\begin{array}{l}r-1,1 \mathrm{a}, 3 \mathrm{a}, c-5,5 \mathrm{a}- \\
\text { pentachlorooctahydro-1,3,4- } \\
\text { metheno-2H-cyclobuta[cd]pentalen- } \\
\text { 2-one }\end{array}$ & chiral \\
\hline 176 & 5 & & $\begin{array}{l}1, r-2,6,8, t-10- \\
\text { pentachloropentacyclo }\left[5 \cdot 3 \cdot 0 \cdot 0^{2,6} \cdot 0^{3,9} \cdot 0^{4,8}\right] \text { decan-5-one }\end{array}$ & $\begin{array}{l}r-1,1 \mathrm{a}, 3 \mathrm{a}, t-5,5 \mathrm{a}- \\
\text { pentachlorooctahydro-1,3,4- } \\
\text { metheno-2H-cyclobuta[cd]pentalen- } \\
\text { 2-one }\end{array}$ & chiral \\
\hline 177 & 5 & & $\begin{array}{l}1,2,3,6,7- \\
\text { pentachloropentacyclo }\left[5 \cdot 3 \cdot 0 \cdot 0^{2,6} \cdot 0^{3,9} \cdot 0^{4,8}\right] \text { decan-5-one }\end{array}$ & $\begin{array}{l}\text { 1,1a,5a,5b,6-pentachlorooctahydro- } \\
\text { 1,3,4-metheno-2H- } \\
\text { cyclobuta[cd]pentalen-2-one }^{b}\end{array}$ & chiral \\
\hline 178 & 5 & & $\begin{array}{l}1,2,3,8,9- \\
\left.\text { pentachloropentacyclo[5.3.0.0 } 0^{2,6} \cdot 0^{3,9} \cdot 0^{4,8}\right] \text { decan-5-one }\end{array}$ & $\begin{array}{l}\text { 1,4,5a,5b,6-pentachlorooctahydro- } \\
\text { 1,3,4-metheno-2H- } \\
\text { cyclobuta[cd]pentalen-2-one }\end{array}$ & chiral \\
\hline
\end{tabular}


Gibbs Free Energy of Formation of Chlordecone and Potential Degradation Products: Implications for Remediation Strategies and Environmental Fate Table SI-1 Structures of all chlordecone potential dechlorinated congeners and their IUPAC and CAS names

\begin{tabular}{|c|c|c|c|c|c|}
\hline Entry & $\overline{\mathrm{nCl}}$ & Structure $^{a}$ & IUPAC - Name & CAS - Name & Chirality \\
\hline 179 & 5 & $\mathbf{H}$ & $\begin{array}{l}1,2,4,8,9- \\
\left.\text { pentachloropentacyclo[5.3.0.0 } 0^{2,6} \cdot 0^{3,9} \cdot 0^{4,8}\right] \text { decan-5-one }\end{array}$ & $\begin{array}{l}\text { 1,3,3a,4,5a-pentachlorooctahydro- } \\
\text { 1,3,4-metheno- } 2 \mathrm{H} \text { - } \\
\text { cyclobuta[cd]pentalen-2-one }^{b}\end{array}$ & chiral \\
\hline 180 & 5 & & $\begin{array}{l}1,2,3,4,8- \\
\left.\text { pentachloropentacyclo[5.3.0.0 } 0^{2,6} \cdot 0^{3,9} \cdot 0^{4,8}\right] \text { decan-5-one }\end{array}$ & $\begin{array}{l}\text { 1,1a,4,5b,6-pentachlorooctahydro- } \\
\text { 1,3,4-metheno-2H- } \\
\text { cyclobuta[cd]pentalen-2-one }\end{array}$ & chiral \\
\hline 181 & 6 & & $\begin{array}{l}1,6,10,10- \\
\left.\text { tetrachloropentacyclo[5.3.0.0 } 0^{2,6} \cdot 0^{3,9} \cdot 0^{4,8}\right] \text { decan-5-one }\end{array}$ & $\begin{array}{l}1^{a}, 5,5,5^{-a}-t e t r a c h l o r o o c t a h y d r o-1,3,4- \\
\text { metheno-2H-cyclobuta[cd]pentalen- } \\
\text { 2-one }\end{array}$ & meso \\
\hline 182 & 6 & & $\begin{array}{l}2,7,10,10- \\
\text { tetrachloropentacyclo }\left[5 \cdot 3 \cdot 0 \cdot 0^{2,6} \cdot 0^{3,9} \cdot 0^{4,8}\right] \text { decan-5-one }\end{array}$ & $\begin{array}{l}\text { 1,5,5,5b-tetrachlorooctahydro-1,3,4- } \\
\text { metheno-2H-cyclobuta[cd]pentalen- } \\
\text { 2-one }\end{array}$ & meso \\
\hline 183 & 6 & & $\begin{array}{l}1,9,10,10- \\
\text { tetrachloropentacyclo }\left[5 \cdot 3 \cdot 0 \cdot 0^{2,6} \cdot 0^{3,9} \cdot 0^{4,8}\right] \text { decan-5-one }\end{array}$ & $\begin{array}{l}\text { 4,5,5,5a-tetrachlorooctahydro-1,3,4- } \\
\text { metheno-2H-cyclobuta[cd]pentalen- } \\
\text { 2-one }\end{array}$ & meso \\
\hline 184 & 6 & & $\begin{array}{l}2,3,10,10- \\
\text { tetrachloropentacyclo }\left[5 \cdot 3 \cdot 0 \cdot 0^{2,6} \cdot 0^{3,9} \cdot 0^{4,8}\right] \text { decan-5-one }\end{array}$ & $\begin{array}{l}\text { 1,5,5,6-tetrachlorooctahydro-1,3,4- } \\
\text { metheno-2H-cyclobuta[cd]pentalen- } \\
\text { 2-one }\end{array}$ & meso \\
\hline
\end{tabular}


Gibbs Free Energy of Formation of Chlordecone and Potential Degradation Products: Implications for Remediation Strategies and Environmental Fate Table SI-1 Structures of all chlordecone potential dechlorinated congeners and their IUPAC and CAS names

\begin{tabular}{|c|c|c|c|c|c|}
\hline Entry & $\overline{\mathrm{nCl}}$ & Structure $^{a}$ & IUPAC - Name & CAS - Name & Chirality \\
\hline 185 & 6 & & $\begin{array}{l}4,6,10,10 \text { - } \\
\left.\text { tetrachloropentacyclo[5.3.0.0 } 0^{2,6} \cdot 0^{3,9} \cdot 0^{4,8}\right] \text { decan-5-one }\end{array}$ & $\begin{array}{l}\text { 1a,3,5,5-tetrachlorooctahydro-1,3,4- } \\
\text { metheno-2H-cyclobuta[cd]pentalen- } \\
\text { 2-one }\end{array}$ & meso \\
\hline 186 & 6 & & $\begin{array}{l}1,4,10,10 \text { - } \\
\left.\text { tetrachloropentacyclo[5.3.0.0 } 0^{2,6} \cdot 0^{3,9} \cdot 0^{4,8}\right] \text { decan-5-one }\end{array}$ & $\begin{array}{l}\text { 1a,4,5,5-tetrachlorooctahydro-1,3,4- } \\
\text { metheno-2H-cyclobuta[cd]pentalen- } \\
\text { 2-one }\end{array}$ & meso \\
\hline 187 & 6 & & $\begin{array}{l}2,4,6,7- \\
\left.\text { tetrachloropentacyclo[5.3.0.0 } 0^{2,6} \cdot 0^{3,9} \cdot 0^{4,8}\right] \text { decan-5-one }\end{array}$ & $\begin{array}{l}\text { 1,1a,3,5b-tetrachlorooctahydro- } \\
\text { 1,3,4-metheno-2H- } \\
\text { cyclobuta[cd]pentalen-2-one }^{\text {b }}\end{array}$ & meso \\
\hline 188 & 6 & & $\begin{array}{l}1,4,6,9- \\
\left.\text { tetrachloropentacyclo[5.3.0.0 } 0^{2,6} \cdot 0^{3,9} \cdot 0^{4,8}\right] \text { decan-5-one }\end{array}$ & $\begin{array}{l}\text { 1a,3,4,5a-tetrachlorooctahydro- } \\
\text { 1,3,4-metheno- } 2 \mathrm{H} \text { - } \\
\text { cyclobuta[cd]pentalen-2-one }\end{array}$ & meso \\
\hline 189 & 6 & & $\begin{array}{l}1,3,6,8- \\
\left.\text { tetrachloropentacyclo[5.3.0.0 } 0^{2,6} \cdot 0^{3,9} \cdot 0^{4,8}\right] \text { decan-5-one }\end{array}$ & $\begin{array}{l}\text { 1,3,4,5b-tetrachlorooctahydro-1,3,4- } \\
\text { metheno-2H-cyclobuta[cd]pentalen- } \\
\text { 2-one }\end{array}$ & meso \\
\hline 190 & 6 & & $\begin{array}{l}2,3,4,6- \\
\text { tetrachloropentacyclo[5.3.0.0 } \\
\left.2,6 \cdot 0^{3,9} \cdot 0^{4,8}\right] \text { decan-5-one }\end{array}$ & $\begin{array}{l}\text { 1,1a,3,6-tetrachlorooctahydro-1,3,4- } \\
\text { metheno-2H-cyclobuta[cd]pentalen- } \\
\text { 2-one }\end{array}$ & meso \\
\hline 191 & 6 & & $\begin{array}{l}1,2,6,7- \\
\text { tetrachloropentacyclo[5.3.0.0 } \\
\left.2,6 \cdot 0^{3,9} \cdot 0^{4,8}\right] \text { decan-5-one }\end{array}$ & $\begin{array}{l}\text { 1,1a,5a,5b-tetrachlorooctahydro- } \\
\text { 1,3,4-metheno-2H- } \\
\text { cyclobuta[cd]pentalen-2-one }^{c}\end{array}$ & meso \\
\hline
\end{tabular}


Gibbs Free Energy of Formation of Chlordecone and Potential Degradation Products: Implications for Remediation Strategies and Environmental Fate Table SI-1 Structures of all chlordecone potential dechlorinated congeners and their IUPAC and CAS names

\begin{tabular}{|c|c|c|c|c|c|}
\hline Entry & $\overline{\mathrm{nCl}}$ & Structure $^{a}$ & IUPAC - Name & CAS - Name & Chirality \\
\hline 192 & 6 & & $\begin{array}{l}1,2,4,7- \\
\text { tetrachloropentacyclo[5.3.0.0 } \\
\left.2,6 \cdot 0^{3,9} \cdot 0^{4,8}\right] \text { decan-5-one }\end{array}$ & $\begin{array}{l}\text { 1,3,5a,5b-tetrachlorooctahydro- } \\
\text { 1,3,4-metheno-2H- } \\
\text { cyclobuta[cd]pentalen-2-one }^{\mathbf{b}}\end{array}$ & meso \\
\hline 193 & 6 & & $\begin{array}{l}1,3,4,8- \\
\text { tetrachloropentacyclo[5.3.0.0 } \\
\left.2,6 \cdot 0^{3,9} \cdot 0^{4,8}\right] \text { decan-5-one }\end{array}$ & $\begin{array}{l}\text { 1,1a,4,5b-tetrachlorooctahydro- } \\
\text { 1,3,4-metheno-2H- } \\
\text { cyclobuta[cd]pentalen-2-one }\end{array}$ & meso \\
\hline 194 & 6 & & $\begin{array}{l}1,2,7,9- \\
\text { tetrachloropentacyclo[5.3.0.0 } \\
\left.2,6 \cdot 0^{3,9} \cdot 0^{4,8}\right] \text { decan-5-one }\end{array}$ & $\begin{array}{l}\text { 1,4,5a,5b-tetrachlorooctahydro- } \\
\text { 1,3,4-metheno-2H- } \\
\text { cyclobuta[cd]pentalen-2-one }^{\text {b }}\end{array}$ & meso \\
\hline 195 & 6 & & $\begin{array}{l}1,2,3,9- \\
\text { tetrachloropentacyclo[5.3.0.0 } \\
\left.2,6 \cdot 0^{3,9} \cdot 0^{4,8}\right] \text { decan-5-one }\end{array}$ & $\begin{array}{l}\text { 1,4,5a,6-tetrachlorooctahydro-1,3,4- } \\
\text { metheno-2H-cyclobuta[cd]pentalen- } \\
\text { 2-one }\end{array}$ & meso \\
\hline 196 & 6 & & $\begin{array}{l}1,2,10,10 \text { - } \\
\left.\text { tetrachloropentacyclo[5.3.0.0 } 0^{2,6} \cdot 0^{3,9} \cdot 0^{4,8}\right] \text { decan-5-one }\end{array}$ & $\begin{array}{l}\text { 1,5,5,5a-tetrachlorooctahydro-1,3,4- } \\
\text { metheno-2H-cyclobuta[cd]pentalen- } \\
\text { 2-one }\end{array}$ & chiral \\
\hline 197 & 6 & & $\begin{array}{l}2,6,10,10 \text { - } \\
\left.\text { tetrachloropentacyclo[5.3.0.0 } 0^{2,6} \cdot 0^{3,9} \cdot 0^{4,8}\right] \text { decan-5-one }\end{array}$ & $\begin{array}{l}\text { 1,1a,5,5-tetrachlorooctahydro-1,3,4- } \\
\text { metheno-2H-cyclobuta[cd]pentalen- } \\
\text { 2-one }\end{array}$ & chiral \\
\hline 198 & 6 & & $\begin{array}{l}1,3,10,10 \text { - } \\
\left.\text { tetrachloropentacyclo[5.3.0.0 } 0^{2,6} \cdot 0^{3,9} \cdot 0^{4,8}\right] \text { decan-5-one }\end{array}$ & $\begin{array}{l}\text { 1,4,5,5-tetrachlorooctahydro-1,3,4- } \\
\text { metheno-2H-cyclobuta[cd]pentalen- } \\
\text { 2-one }\end{array}$ & chiral \\
\hline
\end{tabular}


Gibbs Free Energy of Formation of Chlordecone and Potential Degradation Products: Implications for Remediation Strategies and Environmental Fate Table SI-1 Structures of all chlordecone potential dechlorinated congeners and their IUPAC and CAS names

\begin{tabular}{|c|c|c|c|c|c|}
\hline Entry & $\overline{\mathrm{nCl}}$ & Structure $^{a}$ & IUPAC - Name & CAS - Name & Chirality \\
\hline 199 & 6 & C & $\begin{array}{l}2,4,10,10- \\
\left.\text { tetrachloropentacyclo[5.3.0.0 } 0^{2,6} \cdot 0^{3,9} \cdot 0^{4,8}\right] \text { decan-5-one }\end{array}$ & $\begin{array}{l}\text { 1,3,5,5-tetrachlorooctahydro-1,3,4- } \\
\text { metheno-2H-cyclobuta[cd]pentalen- } \\
\text { 2-one }\end{array}$ & chiral \\
\hline 200 & 6 & & $\begin{array}{l}2,8,10,10 \text { - } \\
\left.\text { tetrachloropentacyclo[5.3.0.0 } 0^{2,6} \cdot 0^{3,9} \cdot 0^{4,8}\right] \text { decan-5-one }\end{array}$ & $\begin{array}{l}\text { 1,3a,5,5-tetrachlorooctahydro-1,3,4- } \\
\text { metheno-2H-cyclobuta[cd]pentalen- } \\
\text { 2-one }\end{array}$ & chiral \\
\hline 201 & 6 & & $\begin{array}{l}1, r-2,6, t-10- \\
\left.\text { tetrachloropentacyclo[5.3.0.0 } 0^{2,6} \cdot 0^{3,9} \cdot 0^{4,8}\right] \text { decan-5-one }\end{array}$ & $\begin{array}{l}r \text {-1,1a,t-5,5a-tetrachlorooctahydro- } \\
\text { 1,3,4-metheno-2H- } \\
\text { cyclobuta[cd]pentalen-2-one }^{\text {b }}\end{array}$ & chiral \\
\hline 202 & 6 & & $\begin{array}{l}1, r-2,6, c-10- \\
\left.\text { tetrachloropentacyclo[5.3.0.0 } 0^{2,6} \cdot 0^{3,9} \cdot 0^{4,8}\right] \text { decan-5-one }\end{array}$ & $\begin{array}{l}r \text {-1,1a,c-5,5a-tetrachlorooctahydro- } \\
\text { 1,3,4-metheno-2H- } \\
\text { cyclobuta[cd]pentalen-2-one }^{\mathbf{b}}\end{array}$ & chiral \\
\hline 203 & 6 & & $\begin{array}{l}2,6,7,10- \\
\left.\text { tetrachloropentacyclo[5.3.0.0 } 0^{2,6} \cdot 0^{3,9} \cdot 0^{4,8}\right] \text { decan-5-one }\end{array}$ & $\begin{array}{l}\text { 1,1a,5,5b-tetrachlorooctahydro- } \\
\text { 1,3,4-metheno- } 2 \mathrm{H}- \\
\text { cyclobuta[cd]pentalen-2-one }^{\mathbf{b}}\end{array}$ & chiral \\
\hline 204 & 6 & & $\begin{array}{l}1,2,7,10- \\
\left.\text { tetrachloropentacyclo[5.3.0.0 } 0^{2,6} \cdot 0^{3,9} \cdot 0^{4,8}\right] \text { decan-5-one }\end{array}$ & $\begin{array}{l}\text { 1,5,5a,5b-tetrachlorooctahydro- } \\
\text { 1,3,4-metheno- } 2 \mathrm{H}- \\
\text { cyclobuta[cd]pentalen-2-one }^{\mathbf{b}}\end{array}$ & chiral \\
\hline 205 & 6 & & $\begin{array}{l}1, r-2,9, t-10- \\
\left.\text { tetrachloropentacyclo[5.3.0.0 } 0^{2,6} \cdot 0^{3,9} \cdot 0^{4,8}\right] \text { decan-5-one }\end{array}$ & $\begin{array}{l}r-1,4, t-5,5 a-t e t r a c h l o r o o c t a h y d r o- \\
\text { 1,3,4-metheno-2H- } \\
\text { cyclobuta[cd]pentalen-2-one }^{b}\end{array}$ & chiral \\
\hline
\end{tabular}


Gibbs Free Energy of Formation of Chlordecone and Potential Degradation Products: Implications for Remediation Strategies and Environmental Fate Table SI-1 Structures of all chlordecone potential dechlorinated congeners and their IUPAC and CAS names

\begin{tabular}{|c|c|c|c|c|c|}
\hline Entry & $\overline{\mathrm{nCl}}$ & Structure $^{a}$ & IUPAC - Name & CAS - Name & Chirality \\
\hline 206 & 6 & & $\begin{array}{l}1, r-2,9, c-10- \\
\left.\text { tetrachloropentacyclo[5.3.0.0 } 0^{2,6} \cdot 0^{3,9} \cdot 0^{4,8}\right] \text { decan-5-one }\end{array}$ & $\begin{array}{l}r \text {-1,4,c-5,5a-tetrachlorooctahydro- } \\
\text { 1,3,4-metheno-2H- } \\
\text { cyclobuta[cd]pentalen-2-one }^{b}\end{array}$ & chiral \\
\hline 207 & 6 & & $\begin{array}{l}1, r-2,3, t-10- \\
\text { tetrachloropentacyclo }\left[5 \cdot 3 \cdot 0 \cdot 0^{2,6} \cdot 0^{3,9} \cdot 0^{4,8}\right] \text { decan-5-one }\end{array}$ & $\begin{array}{l}r \text {-1,4,t-5,6-tetrachlorooctahydro- } \\
\text { 1,3,4-metheno-2H- } \\
\text { cyclobuta[cd]pentalen-2-one }\end{array}$ & chiral \\
\hline 208 & 6 & & $\begin{array}{l}1, r-2,3, c-10- \\
\text { tetrachloropentacyclo }\left[5 \cdot 3 \cdot 0 \cdot 0^{2,6} \cdot 0^{3,9} \cdot 0^{4,8}\right] \text { decan-5-one }\end{array}$ & $\begin{array}{l}r \text {-1,4,c-5,6-tetrachlorooctahydro- } \\
\text { 1,3,4-metheno-2H- } \\
\text { cyclobuta[cd]pentalen-2-one }\end{array}$ & chiral \\
\hline 209 & 6 & & $\begin{array}{l}1, r-2,4, t-10- \\
\left.\text { tetrachloropentacyclo[5.3.0.0 } 0^{2,6} \cdot 0^{3,9} \cdot 0^{4,8}\right] \text { decan-5-one }\end{array}$ & $\begin{array}{l}r-1,3, t-5,5 a-t e t r a c h l o r o o c t a h y d r o- \\
\text { 1,3,4-metheno-2H- } \\
\text { cyclobuta[cd]pentalen-2-one }^{\mathbf{b}}\end{array}$ & chiral \\
\hline 210 & 6 & & $\begin{array}{l}1, r-2,4, c-10- \\
\text { tetrachloropentacyclo }\left[5.3 \cdot 0 \cdot 0^{2,6} \cdot 0^{3,9} \cdot 0^{4,8}\right] \text { decan-5-one }\end{array}$ & $\begin{array}{l}r \text {-1,3,c-5,5a-tetrachlorooctahydro- } \\
\text { 1,3,4-metheno-2H- } \\
\text { cyclobuta[cd]pentalen-2-one }^{b}\end{array}$ & chiral \\
\hline 211 & 6 & & $\begin{array}{l}1, r-2,8, t-10- \\
\left.\text { tetrachloropentacyclo[5.3.0.0 } 0^{2,6} \cdot 0^{3,9} \cdot 0^{4,8}\right] \text { decan-5-one }\end{array}$ & $\begin{array}{l}r \text {-1,3a, } t-5,5 a-t e t r a c h l o r o o c t a h y d r o- \\
\text { 1,3,4-metheno-2H- } \\
\text { cyclobuta[cd]pentalen-2-one }^{\mathbf{b}}\end{array}$ & chiral \\
\hline 212 & 6 & & $\begin{array}{l}1, r-2,8, c-10- \\
\text { tetrachloropentacyclo }\left[5 \cdot 3 \cdot 0 \cdot 0^{2,6} \cdot 0^{3,9} \cdot 0^{4,8}\right] \text { decan-5-one }\end{array}$ & $\begin{array}{l}\text { r-1,3a, c-5,5a-tetrachlorooctahydro- } \\
\text { 1,3,4-metheno-2H- } \\
\text { cyclobuta[cd]pentalen-2-one }^{\text {b }}\end{array}$ & chiral \\
\hline
\end{tabular}


Gibbs Free Energy of Formation of Chlordecone and Potential Degradation Products: Implications for Remediation Strategies and Environmental Fate Table SI-1 Structures of all chlordecone potential dechlorinated congeners and their IUPAC and CAS names

\begin{tabular}{|c|c|c|c|c|c|}
\hline Entry & $\overline{\mathrm{nCl}}$ & Structure $^{a}$ & IUPAC - Name & CAS - Name & Chirality \\
\hline 213 & 6 & & $\begin{array}{l}1, r-3,4, t-10- \\
\left.\text { tetrachloropentacyclo[5.3.0.0 } 0^{2,6} \cdot 0^{3,9} \cdot 0^{4,8}\right] \text { decan-5-one }\end{array}$ & $\begin{array}{l}\text { r-1,1a,4,t-5-tetrachlorooctahydro- } \\
\text { 1,3,4-metheno-2H- } \\
\text { cyclobuta[cd]pentalen-2-one }\end{array}$ & chiral \\
\hline 214 & 6 & & $\begin{array}{l}1, r-3,4, c-10- \\
\left.\text { tetrachloropentacyclo[5.3.0.0 } 0^{2,6} \cdot 0^{3,9} \cdot 0^{4,8}\right] \text { decan-5-one }\end{array}$ & $\begin{array}{l}\text { r-1,1a,4,c-5-tetrachlorooctahydro- } \\
\text { 1,3,4-metheno-2H- } \\
\text { cyclobuta[cd]pentalen-2-one }\end{array}$ & chiral \\
\hline 215 & 6 & & $\begin{array}{l}r-2,3,4, t-10- \\
\left.\text { tetrachloropentacyclo[5.3.0.0 } 0^{2,6} \cdot 0^{3,9} \cdot 0^{4,8}\right] \text { decan-5-one }\end{array}$ & $\begin{array}{l}r \text {-1,1a,t-5,6-tetrachlorooctahydro- } \\
\text { 1,3,4-metheno- } 2 \mathrm{H} \text { - } \\
\text { cyclobuta[cd]pentalen-2-one }\end{array}$ & chiral \\
\hline 216 & 6 & & $\begin{array}{l}r-2,3,4, c-10- \\
\left.\text { tetrachloropentacyclo[5.3.0.0 } 0^{2,6} \cdot 0^{3,9} \cdot 0^{4,8}\right] \text { decan-5-one }\end{array}$ & $\begin{array}{l}\text { r-1,1a,c-5,6-tetrachlorooctahydro- } \\
\text { 1,3,4-metheno-2H- } \\
\text { cyclobuta[cd]pentalen-2-one }\end{array}$ & chiral \\
\hline 217 & 6 & & $\begin{array}{l}r-2,4,6, t-10- \\
\left.\text { tetrachloropentacyclo[5.3.0.0 } 0^{2,6} \cdot 0^{3,9} \cdot 0^{4,8}\right] \text { decan-5-one }\end{array}$ & $\begin{array}{l}\text { r-1,1a,3,t-5-tetrachlorooctahydro- } \\
\text { 1,3,4-metheno-2H- } \\
\text { cyclobuta[cd]pentalen-2-one }^{\mathbf{b}}\end{array}$ & chiral \\
\hline 218 & 6 & & $\begin{array}{l}r-2,4,6, c-10- \\
\text { tetrachloropentacyclo[5.3.0.0 } \\
\left.2,6 \cdot 0^{3,9} \cdot 0^{4,8}\right] \text { decan-5-one }\end{array}$ & $\begin{array}{l}\text { r-1,1a,3,c-5-tetrachlorooctahydro- } \\
\text { 1,3,4-metheno-2H- } \\
\text { cyclobuta[cd]pentalen-2-one }^{\text {b }}\end{array}$ & chiral \\
\hline 219 & 6 & & $\begin{array}{l}r-2,4,8, c-10- \\
\left.\text { tetrachloropentacyclo[5.3.0.0 } 0^{2,6} \cdot 0^{3,9} \cdot 0^{4,8}\right] \text { decan-5-one }\end{array}$ & $\begin{array}{l}r \text {-1,3,3a, } c-5 \text {-tetrachlorooctahydro- } \\
\text { 1,3,4-metheno-2H- } \\
\text { cyclobuta[cd]pentalen-2-one }^{\text {b }}\end{array}$ & chiral \\
\hline
\end{tabular}


Gibbs Free Energy of Formation of Chlordecone and Potential Degradation Products: Implications for Remediation Strategies and Environmental Fate Table SI-1 Structures of all chlordecone potential dechlorinated congeners and their IUPAC and CAS names

\begin{tabular}{|c|c|c|c|c|c|}
\hline Entry & $\overline{\mathrm{nCl}}$ & Structure $^{a}$ & IUPAC - Name & CAS - Name & Chirality \\
\hline 220 & 6 & & $\begin{array}{l}r-2,4,8, t-10- \\
\left.\text { tetrachloropentacyclo[5.3.0.0 } 0^{2,6} \cdot 0^{3,9} \cdot 0^{4,8}\right] \text { decan-5-one }\end{array}$ & $\begin{array}{l}r \text {-1,3,3a, } t-5 \text {-tetrachlorooctahydro- } \\
\text { 1,3,4-metheno-2H- } \\
\text { cyclobuta[cd]pentalen-2-one }^{\mathbf{b}}\end{array}$ & chiral \\
\hline 221 & 6 & & $\begin{array}{l}1,3,8,10 \text { - } \\
\left.\text { tetrachloropentacyclo[5.3.0.0 } 0^{2,6} \cdot 0^{3,9} \cdot 0^{4,8}\right] \text { decan-5-one }\end{array}$ & $\begin{array}{l}\text { 1,4,5,5b-tetrachlorooctahydro-1,3,4- } \\
\text { metheno-2H-cyclobuta[cd]pentalen- } \\
\text { 2-one }\end{array}$ & chiral \\
\hline 222 & 6 & & $\begin{array}{l}r-2,3,7, t-10- \\
\left.\text { tetrachloropentacyclo[5.3.0.0 } 0^{2,6} \cdot 0^{3,9} \cdot 0^{4,8}\right] \text { decan-5-one }\end{array}$ & $\begin{array}{l}r-1,3 \mathrm{a}, \mathrm{c}-5,5 \mathrm{~b} \text {-tetrachlorooctahydro- } \\
\text { 1,3,4-metheno-2H- } \\
\text { cyclobuta[cd]pentalen-2-one }{ }^{c}\end{array}$ & chiral \\
\hline 223 & 6 & & $\begin{array}{l}r-2,3,7, c-10- \\
\left.\text { tetrachloropentacyclo[5.3.0.0 } 0^{2,6} \cdot 0^{3,9} \cdot 0^{4,8}\right] \text { decan-5-one }\end{array}$ & $\begin{array}{l}r-1,3 \mathrm{a}, t-5,5 \mathrm{~b}-\text { tetrachlorooctahydro- } \\
\text { 1,3,4-metheno-2H- } \\
\text { cyclobuta[cd]pentalen-2-one }^{c}\end{array}$ & chiral \\
\hline 224 & 6 & & $\begin{array}{l}2,4,7,10- \\
\left.\text { tetrachloropentacyclo[5.3.0.0 } 0^{2,6} \cdot 0^{3,9} \cdot 0^{4,8}\right] \text { decan-5-one }\end{array}$ & $\begin{array}{l}\text { 1,3,5,5b-tetrachlorooctahydro-1,3,4- } \\
\text { metheno-2H-cyclobuta[cd]pentalen- } \\
\text { 2-one }\end{array}$ & chiral \\
\hline 225 & 6 & & $\begin{array}{l}1,4,9,10 \text { - } \\
\left.\text { tetrachloropentacyclo[5.3.0.0 } 0^{2,6} \cdot 0^{3,9} \cdot 0^{4,8}\right] \text { decan-5-one }\end{array}$ & $\begin{array}{l}\text { 1a,4,5,5a-tetrachlorooctahydro- } \\
\text { 1,3,4-metheno- } 2 \mathrm{H} \text { - } \\
\text { cyclobuta[cd]pentalen-2-one }\end{array}$ & chiral \\
\hline 226 & 6 & & $\begin{array}{l}1, r-3,6, t-10- \\
\left.\text { tetrachloropentacyclo[5.3.0.0 } 0^{2,6} \cdot 0^{3,9} \cdot 0^{4,8}\right] \text { decan-5-one }\end{array}$ & $\begin{array}{l}r-1,3,4, t-5 \text {-tetrachlorooctahydro- } \\
\text { 1,3,4-metheno-2H- } \\
\text { cyclobuta[cd]pentalen-2-one }\end{array}$ & chiral \\
\hline
\end{tabular}


Gibbs Free Energy of Formation of Chlordecone and Potential Degradation Products: Implications for Remediation Strategies and Environmental Fate Table SI-1 Structures of all chlordecone potential dechlorinated congeners and their IUPAC and CAS names

\begin{tabular}{|c|c|c|c|c|c|}
\hline Entry & $\overline{\mathrm{nCl}}$ & Structure $^{\mathrm{a}}$ & IUPAC - Name & CAS - Name & Chirality \\
\hline 227 & 6 & & $\begin{array}{l}1, r-3,6, c-10- \\
\left.\text { tetrachloropentacyclo[5.3.0.0 } 0^{2,6} \cdot 0^{3,9} \cdot 0^{4,8}\right] \text { decan-5-one }\end{array}$ & $\begin{array}{l}r-1,3,4, c-5 \text {-tetrachlorooctahydro- } \\
\text { 1,3,4-metheno-2H- } \\
\text { cyclobuta[cd]pentalen-2-one }\end{array}$ & chiral \\
\hline 228 & 6 & & $\begin{array}{l}1,4,6,10 \text { - } \\
\left.\text { tetrachloropentacyclo[5.3.0.0 } 0^{2,6} \cdot 0^{3,9} \cdot 0^{4,8}\right] \text { decan-5-one }\end{array}$ & $\begin{array}{l}\text { 1a,3,4,5-tetrachlorooctahydro-1,3,4- } \\
\text { metheno-2H-cyclobuta[cd]pentalen- } \\
\text { 2-one }\end{array}$ & chiral \\
\hline 229 & 6 & & $\begin{array}{l}1,2,7,8- \\
\left.\text { tetrachloropentacyclo[5.3.0.0 } 0^{2,6} \cdot 0^{3,9} \cdot 0^{4,8}\right] \text { decan-5-one }\end{array}$ & $\begin{array}{l}\text { 1,3a,5a,5b-tetrachlorooctahydro- } \\
\text { 1,3,4-metheno-2H- } \\
\text { cyclobuta[cd]pentalen-2-one }^{\text {b }}\end{array}$ & chiral \\
\hline 230 & 6 & & $\begin{array}{l}2,3,6,7- \\
\left.\text { tetrachloropentacyclo[5.3.0.0 } 0^{2,6} \cdot 0^{3,9} \cdot 0^{4,8}\right] \text { decan-5-one }\end{array}$ & $\begin{array}{l}\text { 1,1a,5b,6-tetrachlorooctahydro- } \\
\text { 1,3,4-metheno- } 2 \mathrm{H} \text { - } \\
\text { cyclobuta[cd]pentalen-2-one }^{\mathrm{b}}\end{array}$ & chiral \\
\hline 231 & 6 & & $\begin{array}{l}2,4,6,8- \\
\left.\text { tetrachloropentacyclo[5.3.0.0 } 0^{2,6} \cdot 0^{3,9} \cdot 0^{4,8}\right] \text { decan-5-one }\end{array}$ & $\begin{array}{l}1,1^{a}, 3,3^{a}-t e t r a c h l o r o o c t a h y d r o-1,3,4- \\
\text { metheno-2H-cyclobuta[cd]pentalen- } \\
\text { 2-one }\end{array}$ & chiral \\
\hline 232 & 6 & & $\begin{array}{l}1,2,4,8- \\
\left.\text { tetrachloropentacyclo[5.3.0.0 } 0^{2,6} \cdot 0^{3,9} \cdot 0^{4,8}\right] \text { decan-5-one }\end{array}$ & $\begin{array}{l}\text { 1,3,3 }{ }^{\mathrm{a}}, 5^{\mathrm{a}} \text {-tetrachlorooctahydro-1,3,4- } \\
\text { metheno-2H-cyclobuta[cd]pentalen- } \\
\text { 2-one }\end{array}$ & chiral \\
\hline 233 & 6 & & $\begin{array}{l}\text { 1,2,3,4- } \\
\left.\text { tetrachloropentacyclo[5.3.0.0 } 0^{2,6} \cdot 0^{3,9} \cdot 0^{4,8}\right] \text { decan-5-one }\end{array}$ & $\begin{array}{l}\text { 1,1a,4,6-tetrachlorooctahydro-1,3,4- } \\
\text { metheno-2H-cyclobuta[cd]pentalen- } \\
\text { 2-one }\end{array}$ & chiral \\
\hline
\end{tabular}


Gibbs Free Energy of Formation of Chlordecone and Potential Degradation Products: Implications for Remediation Strategies and Environmental Fate Table SI-1 Structures of all chlordecone potential dechlorinated congeners and their IUPAC and CAS names

\begin{tabular}{|c|c|c|c|c|c|}
\hline Entry & $\overline{\mathrm{nCl}}$ & Structure $^{a}$ & IUPAC - Name & CAS - Name & Chirality \\
\hline 234 & 7 & C & $\begin{array}{l}\left.1,10,10 \text {-trichloropentacyclo[5.3.0.0 } 0^{2,6} \cdot 0^{3,9} \cdot 0^{4,8}\right] \text { decan- } \\
\text { 5-one }\end{array}$ & $\begin{array}{l}\text { 4,5,5-trichlorooctahydro-1,3,4- } \\
\text { metheno-2H-cyclobuta[cd]pentalen- } \\
\text { 2-one }\end{array}$ & meso \\
\hline 235 & 7 & & $\begin{array}{l}\left.4,10,10 \text {-trichloropentacyclo[5.3.0.0 } 0^{2,6} \cdot 0^{3,9} \cdot 0^{4,8}\right] \text { decan- } \\
\text { 5-one }\end{array}$ & $\begin{array}{l}\text { 1a,5,5-trichlorooctahydro-1,3,4- } \\
\text { metheno-2H-cyclobuta[cd]pentalen- } \\
\text { 2-one }\end{array}$ & meso \\
\hline 236 & 7 & & $\begin{array}{l}1,9,10 \text {-trichloropentacyclo }\left[5 \cdot 3 \cdot 0 \cdot 0^{2,6} \cdot 0^{3,9} \cdot 0^{4,8}\right] \text { decan-5- } \\
\text { one }\end{array}$ & $\begin{array}{l}\text { 4,5,5a-trichlorooctahydro-1,3,4- } \\
\text { metheno-2H-cyclobuta[cd]pentalen- } \\
\text { 2-one }\end{array}$ & meso \\
\hline 237 & 7 & & $\begin{array}{l}r-2,3, c-10- \\
\left.\text { trichloropentacyclo[5.3.0.0 } 0^{2,6} \cdot 0^{3,9} \cdot 0^{4,8}\right] \text { decan-5-one }\end{array}$ & $\begin{array}{l}r-1, c-5,6 \text {-trichlorooctahydro-1,3,4- } \\
\text { metheno-2H-cyclobuta[cd]pentalen- } \\
\text { 2-one }\end{array}$ & meso \\
\hline 238 & 7 & & $\begin{array}{l}\left.4,6,10 \text {-trichloropentacyclo[5.3.0.0 } 0^{2,6} \cdot 0^{3,9} \cdot 0^{4,8}\right] \text { decan-5- } \\
\text { one }\end{array}$ & $\begin{array}{l}\text { 1a,3,5-trichlorooctahydro-1,3,4- } \\
\text { metheno-2H-cyclobuta[cd]pentalen- } \\
\text { 2-one }\end{array}$ & meso \\
\hline 239 & 7 & & $\begin{array}{l}1,4,6 \text {-trichloropentacyclo }\left[5 \cdot 3 \cdot 0 \cdot 0^{2,6} \cdot 0^{3,9} \cdot 0^{4,8}\right] \text { decan-5- } \\
\text { one }\end{array}$ & $\begin{array}{l}\text { 1a,3,4-trichlorooctahydro-1,3,4- } \\
\text { metheno-2H-cyclobuta[cd]pentalen- } \\
\text { 2-one }\end{array}$ & meso \\
\hline 240 & 7 & & $\begin{array}{l}2,3,10 \text {-trichloropentacyclo }\left[5 \cdot 3 \cdot 0 \cdot 0^{2,6} \cdot 0^{3,9} \cdot 0^{4,8}\right] \text { decan-5- } \\
\text { one }\end{array}$ & $\begin{array}{l}\text { 1,5,6-trichlorooctahydro-1,3,4- } \\
\text { metheno-2H-cyclobuta[cd]pentalen- } \\
\text { 2-one }\end{array}$ & meso \\
\hline
\end{tabular}


Gibbs Free Energy of Formation of Chlordecone and Potential Degradation Products: Implications for Remediation Strategies and Environmental Fate Table SI-1 Structures of all chlordecone potential dechlorinated congeners and their IUPAC and CAS names

\begin{tabular}{|c|c|c|c|c|c|}
\hline Entry & $\overline{\mathrm{nCl}}$ & Structure $^{a}$ & IUPAC - Name & CAS - Name & Chirality \\
\hline 241 & 7 & C & $\begin{array}{l}\left.\text { 1,4,9-trichloropentacyclo[5.3.0.0 } 0^{2,6} \cdot 0^{3,9} \cdot 0^{4,8}\right] \text { decan-5- } \\
\text { one }\end{array}$ & $\begin{array}{l}\text { 1a,4,5a-trichlorooctahydro-1,3,4- } \\
\text { metheno-2H-cyclobuta[cd]pentalen- } \\
\text { 2-one }\end{array}$ & meso \\
\hline 242 & 7 & & $\begin{array}{l}1,3,8 \text {-trichloropentacyclo }\left[5 \cdot 3 \cdot 0 \cdot 0^{2,6} \cdot 0^{3,9} \cdot 0^{4,8}\right] \text { decan-5- } \\
\text { one }\end{array}$ & $\begin{array}{l}\text { 1,4,5b-trichlorooctahydro-1,3,4- } \\
\text { metheno-2H-cyclobuta[cd]pentalen- } \\
\text { 2-one }\end{array}$ & meso \\
\hline 243 & 7 & & $\begin{array}{l}2,4,7 \text {-trichloropentacyclo }\left[5 \cdot 3 \cdot 0 \cdot 0^{2,6} \cdot 0^{3,9} \cdot 0^{4,8}\right] \text { decan-5- } \\
\text { one }\end{array}$ & $\begin{array}{l}\text { 1,3,5b-trichlorooctahydro-1,3,4- } \\
\text { metheno-2H-cyclobuta[cd]pentalen- } \\
\text { 2-one }\end{array}$ & meso \\
\hline 244 & 7 & & $\begin{array}{l}1,2,7 \text {-trichloropentacyclo }\left[5 \cdot 3 \cdot 0 \cdot 0^{2,6} \cdot 0^{3,9} \cdot 0^{4,8}\right] \text { decan-5- } \\
\text { one }\end{array}$ & $\begin{array}{l}\text { 1,5a,5b-trichlorooctahydro-1,3,4- } \\
\text { metheno-2H-cyclobuta[cd]pentalen- } \\
\text { 2-one }\end{array}$ & meso \\
\hline 245 & 7 & & $\begin{array}{l}2,6,7 \text {-trichloropentacyclo }\left[5 \cdot 3 \cdot 0 \cdot 0^{2,6} \cdot 0^{3,9} \cdot 0^{4,8}\right] \text { decan-5- } \\
\text { one }\end{array}$ & $\begin{array}{l}\text { 1,1a,5b-trichlorooctahydro-1,3,4- } \\
\text { metheno-2H-cyclobuta[cd]pentalen- } \\
\text { 2-one }\end{array}$ & meso \\
\hline 246 & 7 & & $\begin{array}{l}2,10,10 \text {-trichloropentacyclo }\left[5 \cdot 3 \cdot 0 \cdot 0^{2,6} \cdot 0^{3,9} \cdot 0^{4,8}\right] \text { decan- } \\
\text { 5-one }\end{array}$ & $\begin{array}{l}\text { 1,5,5-trichlorooctahydro-1,3,4- } \\
\text { metheno-2H-cyclobuta[cd]pentalen- } \\
\text { 2-one }\end{array}$ & chiral \\
\hline 247 & 7 & & $\begin{array}{l}1, r-3, c-10- \\
\text { trichloropentacyclo }\left[5 \cdot 3 \cdot 0 \cdot 0^{2,6} \cdot 0^{3,9} \cdot 0^{4,8}\right] \text { decan-5-one }\end{array}$ & $\begin{array}{l}r-1,4, c-5 \text {-trichlorooctahydro-1,3,4- } \\
\text { metheno-2H-cyclobuta[cd]pentalen- } \\
\text { 2-one }\end{array}$ & chiral \\
\hline
\end{tabular}


Gibbs Free Energy of Formation of Chlordecone and Potential Degradation Products: Implications for Remediation Strategies and Environmental Fate Table SI-1 Structures of all chlordecone potential dechlorinated congeners and their IUPAC and CAS names

\begin{tabular}{|c|c|c|c|c|c|}
\hline Entry & $\overline{\mathrm{nCl}}$ & Structure $^{a}$ & IUPAC - Name & CAS - Name & Chirality \\
\hline 248 & 7 & & $\begin{array}{l}\left.\text { 1,r-3,t-10-trichloropentacyclo[5.3.0.0 } 0^{2,6} \cdot 0^{3,9} \cdot 0^{4,8}\right] \text { decan- } \\
\text { 5-one }\end{array}$ & $\begin{array}{l}r \text {-1,4,t-5-trichlorooctahydro-1,3,4- } \\
\text { metheno-2H-cyclobuta[cd]pentalen- } \\
\text { 2-one }\end{array}$ & chiral \\
\hline 249 & 7 & & $\begin{array}{l}\left.1,4,10 \text {-trichloropentacyclo[5.3.0.0 } 0^{2,6} \cdot 0^{3,9} \cdot 0^{4,8}\right] \text { decan-5- } \\
\text { one }\end{array}$ & $\begin{array}{l}\text { 1a,4,5-trichlorooctahydro-1,3,4- } \\
\text { metheno-2H-cyclobuta[cd]pentalen- } \\
\text { 2-one }\end{array}$ & chiral \\
\hline 250 & 7 & & $\begin{array}{l}r-2,4, c-10- \\
\left.\text { trichloropentacyclo[5.3.0.0 } 0^{2,6} \cdot 0^{3,9} \cdot 0^{4,8}\right] \text { decan-5-one }\end{array}$ & $\begin{array}{l}r \text {-1,3,c-5-trichlorooctahydro-1,3,4- } \\
\text { metheno-2H-cyclobuta[cd]pentalen- } \\
\text { 2-one }\end{array}$ & chiral \\
\hline 251 & 7 & & $\begin{array}{l}r-2,4, t-10 \text {-trichloropentacyclo }\left[5 \cdot 3 \cdot 0 \cdot 0^{2,6} \cdot 0^{3,9} \cdot 0^{4,8}\right] \text { decan- } \\
\text { 5-one }\end{array}$ & $\begin{array}{l}r-1,3, t-5 \text {-trichlorooctahydro-1,3,4- } \\
\text { metheno-2H-cyclobuta[cd]pentalen- } \\
\text { 2-one }\end{array}$ & chiral \\
\hline 252 & 7 & & $\begin{array}{l}\left.2,8,10 \text {-trichloropentacyclo[5.3.0.0 } 0^{2,6} \cdot 0^{3,9} \cdot 0^{4,8}\right] \text { decan-5- } \\
\text { one }\end{array}$ & $\begin{array}{l}\text { 1,3a,5-trichlorooctahydro-1,3,4- } \\
\text { metheno-2H-cyclobuta[cd]pentalen- } \\
\text { 2-one }\end{array}$ & chiral \\
\hline 253 & 7 & & $\begin{array}{l}\text { 1,r-2,c-10- } \\
\left.\text { trichloropentacyclo[5.3.0.0 } 0^{2,6} \cdot 0^{3,9} \cdot 0^{4,8}\right] \text { decan-5-one }\end{array}$ & $\begin{array}{l}r \text {-1,c-5,5a-trichlorooctahydro-1,3,4- } \\
\text { metheno-2H-cyclobuta[cd]pentalen- } \\
\text { 2-one }\end{array}$ & chiral \\
\hline 254 & 7 & & $\begin{array}{l}\left.\text { 1,r-2,t-10-trichloropentacyclo[5.3.0.0 } 0^{2,6} \cdot 0^{3,9} \cdot 0^{4,8}\right] \text { decan- } \\
\text { 5-one }\end{array}$ & $\begin{array}{l}r-1, t-5,5 a-t r i c h l o r o o c t a h y d r o-1,3,4- \\
\text { metheno-2H-cyclobuta[cd]pentalen- } \\
\text { 2-one }\end{array}$ & chiral \\
\hline
\end{tabular}


Gibbs Free Energy of Formation of Chlordecone and Potential Degradation Products: Implications for Remediation Strategies and Environmental Fate Table SI-1 Structures of all chlordecone potential dechlorinated congeners and their IUPAC and CAS names

\begin{tabular}{|c|c|c|c|c|c|}
\hline Entry & $\overline{\mathrm{nCl}}$ & Structure $^{a}$ & IUPAC - Name & CAS - Name & Chirality \\
\hline 255 & 7 & & $\begin{array}{l}\left.1,6,10 \text {-trichloropentacyclo[5.3.0.0 } 0^{2,6} \cdot 0^{3,9} \cdot 0^{4,8}\right] \text { decan-5- } \\
\text { one }\end{array}$ & $\begin{array}{l}\text { 1a,5,5a-trichlorooctahydro-1,3,4- } \\
\text { metheno-2H-cyclobuta[cd]pentalen- } \\
\text { 2-one }\end{array}$ & chiral \\
\hline 256 & 7 & & $\begin{array}{l}\left.2,7,10 \text {-trichloropentacyclo[5.3.0.0 } 0^{2,6} \cdot 0^{3,9} \cdot 0^{4,8}\right] \text { decan-5- } \\
\text { one }\end{array}$ & $\begin{array}{l}\text { 1,5,5b-trichlorooctahydro-1,3,4- } \\
\text { metheno-2H-cyclobuta[cd]pentalen- } \\
\text { 2-one }\end{array}$ & chiral \\
\hline 257 & 7 & & $\begin{array}{l}r-2,6, c-10- \\
\left.\text { trichloropentacyclo[5.3.0.0 } 0^{2,6} \cdot 0^{3,9} \cdot 0^{4,8}\right] \text { decan-5-one }\end{array}$ & $\begin{array}{l}r \text {-1,1a,c-5-trichlorooctahydro-1,3,4- } \\
\text { metheno-2H-cyclobuta[cd]pentalen- } \\
\text { 2-one }\end{array}$ & chiral \\
\hline 258 & 7 & & $\begin{array}{l}r-2,6, t-10 \text {-trichloropentacyclo }\left[5 \cdot 3 \cdot 0 \cdot 0^{2,6} \cdot 0^{3,9} \cdot 0^{4,8}\right] \text { decan- } \\
\text { 5-one }\end{array}$ & $\begin{array}{l}r-1,1 \mathrm{a}, t-5 \text {-trichlorooctahydro-1,3,4- } \\
\text { metheno-2H-cyclobuta[cd]pentalen- } \\
\text { 2-one }\end{array}$ & chiral \\
\hline 259 & 7 & & $\begin{array}{l}\left.\text { 1,3,9-trichloropentacyclo[5.3.0.0 } 0^{2,6} \cdot 0^{3,9} \cdot 0^{4,8}\right] \text { decan-5- } \\
\text { one }\end{array}$ & $\begin{array}{l}\text { 1,4,5a-trichlorooctahydro-1,3,4- } \\
\text { metheno-2H-cyclobuta[cd]pentalen- } \\
\text { 2-one }\end{array}$ & chiral \\
\hline 260 & 7 & & $\begin{array}{l}\text { 1,3,7-trichloropentacyclo }\left[5 \cdot 3 \cdot 0 \cdot 0^{2,6} \cdot 0^{3,9} \cdot 0^{4,8}\right] \text { decan-5- } \\
\text { one }\end{array}$ & $\begin{array}{l}\text { 1,3a,4-trichlorooctahydro-1,3,4- } \\
\text { metheno-2H-cyclobuta[cd]pentalen- } \\
\text { 2-one }\end{array}$ & chiral \\
\hline 261 & 7 & & $\begin{array}{l}1,4,7 \text {-trichloropentacyclo }\left[5 \cdot 3 \cdot 0 \cdot 0^{2,6} \cdot 0^{3,9} \cdot 0^{4,8}\right] \text { decan-5- } \\
\text { one }\end{array}$ & $\begin{array}{l}\text { 1a,3a,4-trichlorooctahydro-1,3,4- } \\
\text { metheno-2H-cyclobuta[cd]pentalen- } \\
\text { 2-one }\end{array}$ & chiral \\
\hline
\end{tabular}


Gibbs Free Energy of Formation of Chlordecone and Potential Degradation Products: Implications for Remediation Strategies and Environmental Fate Table SI-1 Structures of all chlordecone potential dechlorinated congeners and their IUPAC and CAS names

\begin{tabular}{|c|c|c|c|c|c|}
\hline Entry & $\overline{\mathrm{nCl}}$ & Structure $^{a}$ & IUPAC - Name & CAS - Name & Chirality \\
\hline 262 & 7 & & $\begin{array}{l}\left.\text { 1,2,3-trichloropentacyclo[5.3.0.0 } 0^{2,6} \cdot 0^{3,9} \cdot 0^{4,8}\right] \text { decan-5- } \\
\text { one }\end{array}$ & $\begin{array}{l}\text { 1,4,6-trichlorooctahydro-1,3,4- } \\
\text { metheno-2H-cyclobuta[cd]pentalen- } \\
\text { 2-one }\end{array}$ & chiral \\
\hline 263 & 7 & & $\begin{array}{l}\left.\text { 1,3,6-trichloropentacyclo[5.3.0.0 } 0^{2,6} \cdot 0^{3,9} \cdot 0^{4,8}\right] \text { decan-5- } \\
\text { one }\end{array}$ & $\begin{array}{l}\text { 1,3,4-trichlorooctahydro-1,3,4- } \\
\text { metheno-2H-cyclobuta[cd]pentalen- } \\
\text { 2-one }\end{array}$ & chiral \\
\hline 264 & 7 & & $\begin{array}{l}\left.2,3,7 \text {-trichloropentacyclo[5.3.0.0 } 0^{2,6} \cdot 0^{3,9} \cdot 0^{4,8}\right] \text { decan-5- } \\
\text { one }\end{array}$ & $\begin{array}{l}\text { 1,3a,5b-trichlorooctahydro-1,3,4- } \\
\text { metheno-2H-cyclobuta[cd]pentalen- } \\
\text { 2-one }{ }^{c}\end{array}$ & chiral \\
\hline 265 & 7 & & $\begin{array}{l}\left.\text { 1,3,4-trichloropentacyclo[5.3.0.0 } 0^{2,6} \cdot 0^{3,9} \cdot 0^{4,8}\right] \text { decan-5- } \\
\text { one }\end{array}$ & $\begin{array}{l}\text { 1,1a,4-trichlorooctahydro-1,3,4- } \\
\text { metheno-2H-cyclobuta[cd]pentalen- } \\
\text { 2-one }\end{array}$ & chiral \\
\hline 266 & 7 & & $\begin{array}{l}2,4,8 \text {-trichloropentacyclo }\left[5 \cdot 3 \cdot 0 \cdot 0^{2,6} \cdot 0^{3,9} \cdot 0^{4,8}\right] \text { decan-5- } \\
\text { one }\end{array}$ & $\begin{array}{l}\text { 1,1a,3a-trichlorooctahydro-1,3,4- } \\
\text { metheno-2H-cyclobuta[cd]pentalen- } \\
\text { 2-one }\end{array}$ & chiral \\
\hline 267 & 7 & & $\begin{array}{l}2,4,6 \text {-trichloropentacyclo }\left[5 \cdot 3 \cdot 0 \cdot 0^{2,6} \cdot 0^{3,9} \cdot 0^{4,8}\right] \text { decan-5- } \\
\text { one }\end{array}$ & $\begin{array}{l}\text { 1,1a,3-trichlorooctahydro-1,3,4- } \\
\text { metheno-2H-cyclobuta[cd]pentalen- } \\
\text { 2-one }\end{array}$ & chiral \\
\hline 268 & 7 & & $\begin{array}{l}2,3,4 \text {-trichloropentacyclo }\left[5 \cdot 3 \cdot 0 \cdot 0^{2,6} \cdot 0^{3,9} \cdot 0^{4,8}\right] \text { decan-5- } \\
\text { one }\end{array}$ & $\begin{array}{l}\text { 1,1a,6-trichlorooctahydro-1,3,4- } \\
\text { metheno-2H-cyclobuta[cd]pentalen- } \\
\text { 2-one }\end{array}$ & chiral \\
\hline
\end{tabular}


Gibbs Free Energy of Formation of Chlordecone and Potential Degradation Products: Implications for Remediation Strategies and Environmental Fate Table SI-1 Structures of all chlordecone potential dechlorinated congeners and their IUPAC and CAS names

\begin{tabular}{|c|c|c|c|c|c|}
\hline Entry & $\overline{\mathrm{nCl}}$ & Structure $^{\mathrm{a}}$ & IUPAC - Name & CAS - Name & Chirality \\
\hline 269 & 7 & & $\begin{array}{l}\left.1,2,6 \text {-trichloropentacyclo[5.3.0.0 } 0^{2,6} \cdot 0^{3,9} \cdot 0^{4,8}\right] \text { decan-5- } \\
\text { one }\end{array}$ & $\begin{array}{l}\text { 1,1a,5a-trichlorooctahydro-1,3,4- } \\
\text { metheno-2H-cyclobuta[cd]pentalen- } \\
\text { 2-one }\end{array}$ & chiral \\
\hline 270 & 8 & & $\begin{array}{l}\left.10,10 \text {-bichloropentacyclo[5.3.0.0 } 0^{2,6} \cdot 0^{3,9} \cdot 0^{4,8}\right] \text { decan-5- } \\
\text { one }\end{array}$ & $\begin{array}{l}\text { 5,5-bichlorooctahydro-1,3,4- } \\
\text { metheno-2H-cyclobuta[cd]pentalen- } \\
\text { 2-one }\end{array}$ & meso \\
\hline 271 & 8 & & 1,6-bichloropentacyclo[5.3.0.0 $\left.0^{2,6} \cdot 0^{3,9} \cdot 0^{4,8}\right]$ decan-5-one & $\begin{array}{l}\text { 1a,5a-bichlorooctahydro-1,3,4- } \\
\text { metheno-2H-cyclobuta[cd]pentalen- } \\
\text { 2-one }{ }^{c}\end{array}$ & meso \\
\hline 272 & 8 & & 2,7-bichloropentacyclo[5.3.0.0 $\left.0^{2,6} \cdot 0^{3,9} \cdot 0^{4,8}\right]$ decan-5-one & $\begin{array}{l}\text { 1,5b-bichlorooctahydro-1,3,4- } \\
\text { metheno-2H-cyclobuta[cd]pentalen- } \\
\text { 2-one }\end{array}$ & meso \\
\hline 273 & 8 & & 1,9-bichloropentacyclo[5.3.0.0 $\left.0^{2,6} \cdot 0^{3,9} \cdot 0^{4,8}\right]$ decan-5-one & $\begin{array}{l}\text { 4,5a-bichlorooctahydro-1,3,4- } \\
\text { metheno-2H-cyclobuta[cd]pentalen- } \\
\text { 2-one }\end{array}$ & meso \\
\hline 274 & 8 & & 1,4-bichloropentacyclo[5.3.0.0 $\left.0^{2,6} \cdot 0^{3,9} \cdot 0^{4,8}\right]$ decan-5-one & $\begin{array}{l}\text { 1a,4-bichlorooctahydro-1,3,4- } \\
\text { metheno-2H-cyclobuta[cd]pentalen- } \\
\text { 2-one }\end{array}$ & meso \\
\hline 275 & 8 & & 2,3-bichloropentacyclo[5.3.0.0.0.6. $\left.\cdot 0^{3,9} \cdot 0^{4,8}\right]$ decan-5-one & $\begin{array}{l}\text { 1,6-bichlorooctahydro-1,3,4- } \\
\text { metheno-2H-cyclobuta[cd]pentalen- } \\
\text { 2-one }\end{array}$ & meso \\
\hline
\end{tabular}


Gibbs Free Energy of Formation of Chlordecone and Potential Degradation Products: Implications for Remediation Strategies and Environmental Fate Table SI-1 Structures of all chlordecone potential dechlorinated congeners and their IUPAC and CAS names

\begin{tabular}{|c|c|c|c|c|c|}
\hline Entry & $\overline{\mathrm{nCl}}$ & Structure $^{a}$ & IUPAC - Name & CAS - Name & Chirality \\
\hline 276 & 8 & & 4,6-bichloropentacyclo[5.3.0.0 $\left.0^{2,6} \cdot 0^{3,9} \cdot 0^{4,8}\right]$ decan-5-one & $\begin{array}{l}\text { 1a,3-bichlorooctahydro-1,3,4- } \\
\text { metheno-2H-cyclobuta[cd]pentalen- } \\
\text { 2-one }\end{array}$ & meso \\
\hline 277 & 8 & & $\begin{array}{l}\left.\text { 1,10-bichloropentacyclo[5.3.0.0 } 0^{2,6} \cdot 0^{3,9} \cdot 0^{4,8}\right] \text { decan-5- } \\
\text { one }\end{array}$ & $\begin{array}{l}\text { 4,5-bichlorooctahydro-1,3,4- } \\
\text { metheno-2H-cyclobuta[cd]pentalen- } \\
\text { 2-one }\end{array}$ & chiral \\
\hline 278 & 8 & & $\begin{array}{l}\left.r-2, c-10 \text {-bichloropentacyclo[5.3.0.0 } 0^{2,6} \cdot 0^{3,9} \cdot 0^{4,8}\right] \text { decan- } \\
\text { 5-one }\end{array}$ & $\begin{array}{l}r \text {-1,c-5-bichlorooctahydro-1,3,4- } \\
\text { metheno-2H-cyclobuta[cd]pentalen- } \\
\text { 2-one }\end{array}$ & chiral \\
\hline 279 & 8 & & $\begin{array}{l}r-2, t-10 \text {-bichloropentacyclo }\left[5 \cdot 3 \cdot 0 \cdot 0^{2,6} \cdot 0^{3,9} \cdot 0^{4,8}\right] \text { decan-5- } \\
\text { one }\end{array}$ & $\begin{array}{l}r \text {-1,t-5-bichlorooctahydro-1,3,4- } \\
\text { metheno-2H-cyclobuta[cd]pentalen- } \\
\text { 2-one }\end{array}$ & chiral \\
\hline 280 & 8 & & $\begin{array}{l}4,10 \text {-bichloropentacyclo }\left[5 \cdot 3 \cdot 0 \cdot 0^{2,6} \cdot 0^{3,9} \cdot 0^{4,8}\right] \text { decan-5- } \\
\text { one }\end{array}$ & $\begin{array}{l}\text { 1a,5-bichlorooctahydro-1,3,4- } \\
\text { metheno-2H-cyclobuta[cd]pentalen- } \\
\text { 2-one }\end{array}$ & chiral \\
\hline 281 & 8 & & 1,2-bichloropentacyclo[5.3.0.0 $\left.0^{2,6} \cdot 0^{3,9} \cdot 0^{4,8}\right]$ decan-5-one & $\begin{array}{l}\text { 1,5a-bichlorooctahydro-1,3,4- } \\
\text { metheno-2H-cyclobuta[cd]pentalen- } \\
\text { 2-one }\end{array}$ & chiral \\
\hline 282 & 8 & & 2,6-bichloropentacyclo[5.3.0.0 $\left.0^{2,6} \cdot 0^{3,9} \cdot 0^{4,8}\right]$ decan-5-one & $\begin{array}{l}\text { 1,1a-bichlorooctahydro-1,3,4- } \\
\text { metheno-2H-cyclobuta[cd]pentalen- } \\
\text { 2-one }\end{array}$ & chiral \\
\hline
\end{tabular}


Gibbs Free Energy of Formation of Chlordecone and Potential Degradation Products: Implications for Remediation Strategies and Environmental Fate Table SI-1 Structures of all chlordecone potential dechlorinated congeners and their IUPAC and CAS names

\begin{tabular}{|c|c|c|c|c|c|}
\hline Entry & $\overline{\mathrm{nCl}}$ & Structure $^{a}$ & IUPAC - Name & CAS - Name & Chirality \\
\hline 283 & 8 & & 1,3-bichloropentacyclo[5.3.0.0 $\left.0^{2,6} \cdot 0^{3,9} \cdot 0^{4,8}\right]$ decan-5-one & $\begin{array}{l}\text { 1,4-bichlorooctahydro-1,3,4- } \\
\text { metheno-2H-cyclobuta[cd]pentalen- } \\
\text { 2-one }\end{array}$ & chiral \\
\hline 284 & 8 & & 2,4-bichloropentacyclo[5.3.0.0 $\left.0^{2,6} \cdot 0^{3,9} \cdot 0^{4,8}\right]$ decan-5-one & $\begin{array}{l}\text { 1,3-bichlorooctahydro-1,3,4- } \\
\text { metheno-2H-cyclobuta[cd]pentalen- } \\
\text { 2-one }\end{array}$ & chiral \\
\hline 285 & 8 & & 2,8-bichloropentacyclo[5.3.0.0 $\left.0^{2,6} \cdot 0^{3,9} \cdot 0^{4,8}\right]$ decan-5-one & $\begin{array}{l}\text { 1,3a-bichlorooctahydro-1,3,4- } \\
\text { metheno-2H-cyclobuta[cd]pentalen- } \\
\text { 2-one }\end{array}$ & chiral \\
\hline 286 & 9 & & $\begin{array}{l}\left.10 \text {-monochloropentacyclo[5.3.0.0 } 0^{2,6} \cdot 0^{3,9} \cdot 0^{4,8}\right] \text { decan-5- } \\
\text { one }\end{array}$ & $\begin{array}{l}\text { 5-monochlorooctahydro-1,3,4- } \\
\text { metheno-2H-cyclobuta[cd]pentalen- } \\
\text { 2-one }\end{array}$ & meso \\
\hline 287 & 9 & & $\begin{array}{l}\text { 1-monochloropentacyclo }\left[5 \cdot 3 \cdot 0 \cdot 0^{2,6} \cdot 0^{3,9} \cdot 0^{4,8}\right] \text { decan-5- } \\
\text { one }\end{array}$ & $\begin{array}{l}\text { 4-monochlorooctahydro-1,3,4- } \\
\text { metheno-2H-cyclobuta[cd]pentalen- } \\
\text { 2-one }\end{array}$ & meso \\
\hline 288 & 9 & & $\begin{array}{l}\text { 4-monochloropentacyclo }\left[5 \cdot 3 \cdot 0 \cdot 0^{2,6} \cdot 0^{3,9} \cdot 0^{4,8}\right] \text { decan-5- } \\
\text { one }\end{array}$ & $\begin{array}{l}\text { 1a-monochlorooctahydro-1,3,4- } \\
\text { metheno-2H-cyclobuta[cd]pentalen- } \\
\text { 2-one }\end{array}$ & meso \\
\hline 289 & 9 & & $\begin{array}{l}\text { 2-monochloropentacyclo }\left[5 \cdot 3 \cdot 0 \cdot 0^{2,6} \cdot 0^{3,9} \cdot 0^{4,8}\right] \text { decan-5- } \\
\text { one }\end{array}$ & $\begin{array}{l}\text { 1-monochlorooctahydro-1,3,4- } \\
\text { metheno-2H-cyclobuta[cd]pentalen- } \\
\text { 2-one }\end{array}$ & chiral \\
\hline
\end{tabular}


Gibbs Free Energy of Formation of Chlordecone and Potential Degradation Products: Implications for Remediation Strategies and Environmental Fate Table SI-1 Structures of all chlordecone potential dechlorinated congeners and their IUPAC and CAS names

\begin{tabular}{|c|c|c|c|c|c|}
\hline Entry & $\overline{\mathrm{nCl}}$ & Structure $^{a}$ & IUPAC - Name & CAS - Name & Chirality \\
\hline 290 & 10 & & pentacyclo[5.3.0.0 $\left.0^{2,6} \cdot 0^{3,9} \cdot 0^{4,8}\right]$ decan-5-one & $\begin{array}{l}\text { octahydro-1,3,4-metheno-2H- } \\
\text { cyclobuta[cd]pentalen-2-one }\end{array}$ & meso \\
\hline
\end{tabular}

a - The structures represented in the Table corresponds to the IUPAC numerotation.

$\mathrm{b}$ - The CAS name corresponds to the enantiomer of the IUPAC structure represented in the same line

$\mathrm{c}$ - The CAS name corresponds to a $\mathrm{C}_{2}$ rotation $\left(180^{\circ}\right)$ around the carbonyl bound, of the IUPAC structure represented in the same line. 
Gibbs Free Energy of Formation of Chlordecone and Potential Degradation Products: Implications for Remediation Strategies and Environmental Fate

Table SI-2. Calculated $\Delta_{\mathrm{f}} G^{0}, \Delta_{\mathrm{f}} H^{0}$, and $S^{0}$ data for 62 chlordecone congeners plus corresponding Henry constants, strain energy (SE) and dipole $(\mu)$ values $^{a}$

\begin{tabular}{|c|c|c|c|c|c|c|c|c|c|c|}
\hline Compound & $\begin{array}{l}\text { Entry in } \\
\text { Table SI-1 }\end{array}$ & $\begin{array}{c}\Delta_{\mathrm{f}} H^{\circ}{ }_{\text {gas }} \\
(\mathrm{kJ} / \mathrm{mol})\end{array}$ & $\begin{array}{c}S^{\circ} \text { gas } \\
(\mathrm{J} / \mathrm{mol} . \mathrm{K})\end{array}$ & $\begin{array}{l}\Delta_{\mathrm{f}} G_{\text {gas }}^{\circ} \\
(\mathrm{kJ} / \mathrm{mol})\end{array}$ & $\begin{array}{l}\Delta_{\mathrm{f}} H_{\text {aqueous }}^{\circ} \\
(\mathrm{kJ} / \mathrm{mol})\end{array}$ & $\begin{array}{l}S^{\circ}{ }_{\text {aqueous }} \\
(\mathrm{J} / \mathrm{mol} . \mathrm{K})\end{array}$ & $\begin{array}{c}\Delta_{f} G_{\text {aqueous }}^{\circ} \\
(\mathrm{kJ} / \mathrm{mol})\end{array}$ & $\begin{array}{c}\text { SE } \\
(\mathrm{kJ} / \mathrm{mol})\end{array}$ & $\begin{array}{l}\mu \\
\mathrm{D}\end{array}$ & $\begin{array}{c}H \\
\text { (atm.L/mol) }\end{array}$ \\
\hline chlordecone & 1 & -225.9 & 764 & -73.5 & -244.6 & 764 & -92.0 & 483.7 & 1.78 & $5.76 \mathrm{E}-04$ \\
\hline chlordecol & NA & -332.8 & 771 & -143.5 & -378.0 & 682 & -162.3 & 433.4 & 2.24 & $5.10 \mathrm{E}-04$ \\
\hline 10-monohydrochlordecone & 2 & -199.2 & 736 & -52.3 & -226.0 & 737 & -79.2 & 478.7 & 2.44 & $1.95 \mathrm{E}-05$ \\
\hline 9-monohydrochlordecone & 3 & -201.8 & 735 & -54.6 & -227.5 & 735 & -80.1 & 455.7 & 2.37 & $3.42 \mathrm{E}-05$ \\
\hline 8-monohydrochlordecone* & 5 & -201.6 & 736 & -54.5 & -227.8 & 735 & -80.5 & 455.9 & 1.97 & $2.80 \mathrm{E}-05$ \\
\hline 6-monohydrochlordecone & 4 & -205.4 & 734 & -57.8 & -230.0 & 714 & -76.3 & 452.1 & 1.68 & $5.66 \mathrm{E}-04$ \\
\hline 10,10-dihydrochlordecone & 6 & -176.4 & 707 & -34.5 & -200.3 & 706 & -58.1 & 458.4 & 3.45 & $7.36 \mathrm{E}-05$ \\
\hline 9,10-dihydrochlordecone* & 13 & -174.7 & 707 & -32.9 & -207.4 & 706 & -65.2 & 451.0 & 3.15 & $2.20 \mathrm{E}-06$ \\
\hline cis-8,10-dihydrochlordecone* & 14 & -173.3 & 708 & -31.7 & -209.0 & 706 & -66.9 & 452.4 & 3.00 & $6.85 \mathrm{E}-07$ \\
\hline trans-8,10-dihydrochlordecone* & 15 & -174.8 & 708 & -33.2 & -207.9 & 707 & -66.1 & 451.0 & 2.28 & $1.73 \mathrm{E}-06$ \\
\hline 8,9-dihydrochlordecone* & 17 & -178.1 & 706 & -35.9 & -210.2 & 706 & -67.9 & 427.3 & 2.85 & $2.49 \mathrm{E}-06$ \\
\hline 7,9-dihydrochlordecone* & 19 & -177.1 & 710 & -36.0 & -211.1 & 692 & -64.6 & 428.3 & 2.39 & $9.76 \mathrm{E}-06$ \\
\hline 7,8-dihydrochlordecone & 11 & -171.3 & 705 & -28.8 & -205.7 & 705 & -63.1 & 434.1 & 2.71 & $1.00 \mathrm{E}-06$ \\
\hline 6,10-dihydrochlordecone* & 16 & -178.7 & 706 & -36.6 & -211.5 & 705 & -69.1 & 447.0 & 2.30 & $2.03 \mathrm{E}-06$ \\
\hline 6,9-dihydrochlordecone & 10 & -180.7 & 705 & -38.3 & -211.6 & 705 & -69.1 & 424.7 & 1.67 & 4.04E-06 \\
\hline 6,8-dihydrochlordecone* & 20 & -180.7 & 706 & -38.4 & -212.1 & 705 & -69.7 & 424.7 & 1.68 & 3.30E-06 \\
\hline 6,7-dihydrochlordecone* & 18 & -181.4 & 704 & -38.7 & -213.2 & 704 & -70.4 & 424.0 & 2.30 & $2.81 \mathrm{E}-06$ \\
\hline 4,9-dihydrochlordecone & 7 & -177.6 & 705 & -35.1 & -210.9 & 704 & -68.2 & 427.8 & 2.76 & $1.60 \mathrm{E}-06$ \\
\hline 4,6-dihydrochlordecone & 12 & -182.6 & 704 & -39.7 & -211.9 & 704 & -69.0 & 422.9 & 0.72 & $7.39 \mathrm{E}-06$ \\
\hline 3,8-dihydrochlordecone & 8 & -173.2 & 707 & -31.3 & -193.4 & 707 & -51.4 & 432.3 & 1.86 & $3.02 \mathrm{E}-04$ \\
\hline 3,7-dihydrochlordecone* & 21 & -177.9 & 708 & -36.2 & -210.7 & 707 & -68.8 & 427.6 & 1.69 & $1.95 \mathrm{E}-06$ \\
\hline 1,9-dihydrochlordecone & 9 & -175.5 & 706 & -33.4 & -206.7 & 705 & -64.3 & 429.9 & 2.61 & $3.88 \mathrm{E}-06$ \\
\hline 8,10,10-trihydrochlordecone* & 34 & -150.0 & 678 & -13.3 & -180.7 & 677 & -43.7 & 432.7 & 3.65 & 4.74E-06 \\
\hline 3,7,10-trihydrochlordecone* & 40 & -149.6 & 679 & -13.2 & -190.2 & 678 & -53.5 & 424.1 & 2.47 & $8.75 \mathrm{E}-08$ \\
\hline 3,7,10,10-tetrahydrochlordecone* & 77 & -123.5 & 647 & 8.8 & -159.9 & 646 & -27.4 & 407.1 & 3.50 & 4.57E-07 \\
\hline $\mathrm{r}-3,7,8, \mathrm{t}-10$-tetrahydrochlordecone* & 100 & -111.6 & 647 & 20.8 & -158.7 & 646 & -26.2 & 410.0 & 3.43 & 5.99E-09 \\
\hline $3,7,8,10,10$-pentahydrochlordecone* & 134 & -84.8 & 614 & 43.5 & -125.9 & 614 & 2.5 & 393.8 & 3.89 & $6.60 \mathrm{E}-08$ \\
\hline $2,3,7,8,10,10$-hexahydrochlordecone & 188 & -45.1 & 582 & 79.1 & -89.2 & 582 & 34.9 & 381.3 & 3.57 & $1.82 \mathrm{E}-08$ \\
\hline $3,4,7,8,9,10,10$-heptahydrochlordecone* & 269 & -23.9 & 546 & 97.0 & -52.4 & 546 & 68.5 & 277.8 & 6.09 & $1.04 \mathrm{E}-05$ \\
\hline $\mathrm{r}-3,4,6,7,8,9, \mathrm{c}-10$-heptahydrochlordecone* & 254 & -31.5 & 548 & 88.8 & -56.4 & 549 & 63.7 & 333.8 & 3.07 & 4.07E-05 \\
\hline $2,6,7,8,9,10,10$-heptahydrochlordecone* & 265 & -30.2 & 548 & 90.2 & -54.6 & 548 & 65.8 & 271.5 & 4.23 & $5.40 \mathrm{E}-05$ \\
\hline$r-2,4,6,7,8,9, c-10$-heptahydrochlordecone* & 248 & -33.0 & 550 & 86.9 & -56.7 & 550 & 63.1 & 332.4 & 1.78 & $6.65 \mathrm{E}-05$ \\
\hline 2,3,7,8,9,10,10-heptahydrochlordecone & 239 & -14.0 & 548 & 106.4 & -56.4 & 548 & 64.0 & 360.4 & 4.96 & 3.75E-08 \\
\hline $1,6,7,8,9,10,10$-heptahydrochlordecone* & 268 & -26.7 & 546 & 94.3 & -52.9 & 545 & 68.4 & 274.9 & 6.09 & $2.91 \mathrm{E}-05$ \\
\hline $1,3,7,8,9,10,10$-heptahydrochlordecone* & 267 & -23.7 & 548 & 96.8 & -48.3 & 548 & 72.1 & 278.0 & 5.85 & 4.74E-05 \\
\hline $1,3,6,7,9,10,10$-heptahydrochlordecone* & 266 & -36.5 & 548 & 83.8 & -60.6 & 549 & 59.7 & 265.2 & 5.00 & $6.02 \mathrm{E}-05$ \\
\hline 1,3,4,8,9,10,10-heptahydrochlordecone & 245 & -30.4 & 547 & 90.4 & -54.8 & 547 & 66.0 & 271.3 & 5.78 & $5.22 \mathrm{E}-05$ \\
\hline $1,3,4,6,8,9,10$-Heptahydrochlordecone* & 256 & -32.3 & 551 & 87.4 & -57.1 & 551 & 62.4 & 333.0 & 3.26 & 4.25E-05 \\
\hline 1,3,4,6,7,9,10-heptahydrochlordecone* & 252 & -37.1 & 551 & 82.5 & -63.0 & 551 & 56.5 & 328.2 & 2.62 & $2.77 \mathrm{E}-05$ \\
\hline 1,3,4,6,7,8,9-heptahydrochlordecone* & 246 & -27.6 & 547 & 93.1 & -51.2 & 548 & 69.2 & 337.8 & 2.37 & $6.55 \mathrm{E}-05$ \\
\hline $1, r-3,4,7,8,9, c-10$-heptahydrochlordecone* & 258 & -27.7 & 549 & 92.3 & -53.5 & 550 & 66.5 & 337.6 & 3.97 & 2.97E-05 \\
\hline $3,4,6,7,8,9,10,10$-octahydrochlordecone* & 281 & 3.5 & 514 & 120.4 & -36.0 & 515 & 80.6 & 325.8 & 4.72 & $1.08 \mathrm{E}-07$ \\
\hline $2,4,6,7,8,9,10,10$-octahydrochlordecone & 283 & 1.5 & 515 & 117.9 & -37.5 & 516 & 78.7 & 323.8 & 3.87 & $1.35 \mathrm{E}-07$ \\
\hline $2,3,6,7,8,9,10,10$-octahydrochlordecone & 274 & 13.7 & 514 & 130.4 & -9.5 & 515 & 107.1 & 336.0 & 3.88 & $8.31 \mathrm{E}-05$ \\
\hline $2,3,4,7,8,9,10,10$-octahydrochlordecone & 271 & 19.3 & 514 & 136.2 & -23.4 & 514 & 93.4 & 341.6 & 5.58 & $3.17 \mathrm{E}-08$ \\
\hline $2,3,4,6,7,8,10,10$-octahydrochlordecone & 273 & 7.3 & 515 & 123.9 & -31.2 & 515 & 85.2 & 329.6 & 2.06 & $1.67 \mathrm{E}-07$ \\
\hline $2,3,4,6,7,8,9,10$-octahydrochlordecone* & 277 & 12.3 & 515 & 128.8 & -31.8 & 516 & 84.4 & 325.6 & 2.82 & $1.70 \mathrm{E}-08$ \\
\hline 1,4,6,7,8,9,10,10-octahydrochlordecone & 275 & 5.7 & 513 & 122.7 & -34.4 & 514 & 82.3 & 328.0 & 5.51 & $8.51 \mathrm{E}-08$ \\
\hline $1,3,6,7,8,9,10,10$-octahydrochlordecone* & 284 & 8.1 & 515 & 124.7 & -30.8 & 515 & 85.6 & 330.4 & 5.40 & $1.42 \mathrm{E}-07$ \\
\hline $1,3,4,6,8,9,10,10$-octahydrochlordecone & 272 & 0.2 & 515 & 116.6 & -37.1 & 516 & 79.1 & 322.5 & 4.54 & 2.69E-07 \\
\hline $1, r-3,4,6,7,8,9, t-10$-octahydrochlordecone* & 278 & 8.9 & 516 & 125.1 & -37.9 & 517 & 78.1 & 322.2 & 4.28 & $5.80 \mathrm{E}-09$ \\
\hline $1, r-3,4,6,7,8,9, c-10$-octahydrochlordecone* & 279 & 4.8 & 517 & 120.8 & -18.1 & 517 & 97.7 & 318.0 & 2.44 & $9.00 \mathrm{E}-05$ \\
\hline 1,3,4,7,8,9,10,10-octahydrochlordecone* & 282 & 9.6 & 514 & 126.5 & -29.7 & 514 & 87.0 & 331.9 & 5.91 & $1.21 \mathrm{E}-07$ \\
\hline $1,3,4,6,7,9,10,10$-octahydrochlordecone* & 285 & -4.8 & 515 & 111.6 & -26.6 & 516 & 89.6 & 317.6 & 4.04 & $1.40 \mathrm{E}-04$ \\
\hline $1,2,3,7,8,9,10,10$-octahydrochlordecone & 276 & 20.4 & 514 & 137.1 & -17.7 & 515 & 98.9 & 342.7 & 5.70 & $2.04 \mathrm{E}-07$ \\
\hline $1,2,3,6,7,8,9,10$-octahydrochlordecone* & 280 & 16.5 & 516 & 132.8 & -27.9 & 517 & 88.2 & 329.8 & 3.96 & $1.53 \mathrm{E}-08$ \\
\hline $1,2,3,4,6,7,8,9$-octahydrochlordecone & 270 & 15.7 & 513 & 132.7 & -24.0 & 514 & 92.7 & 308.7 & 1.01 & $1.00 \mathrm{E}-07$ \\
\hline $2,3,4,6,7,8,9,10,10$-nonahydrochlordecone & 287 & 49.0 & 480 & 162.1 & 12.6 & 481 & 125.4 & 319.3 & 3.82 & $3.71 \mathrm{E}-07$ \\
\hline $1,3,4,6,7,8,9,10,10$-nonahydrochlordecone* & 289 & 43.3 & 481 & 156.2 & 6.8 & 482 & 119.4 & 313.5 & 4.79 & $3.59 \mathrm{E}-07$ \\
\hline 1,2,3,6,7,8,9,10,10-nonahydrochlordecone & 288 & 55.9 & 483 & 168.3 & 19.6 & 483 & 131.8 & 289.8 & 5.42 & 4.05E-07 \\
\hline $1,2,3,4,6,7,8,9,10$-nonahydrochlordecone & 286 & 52.6 & 482 & 165.3 & 11.9 & 483 & 124.2 & 313.8 & 2.70 & $6.44 \mathrm{E}-08$ \\
\hline decahydrochlordecone & 290 & 94.2 & 446 & 203.8 & 62.5 & 446 & 171.9 & 312.3 & 4.18 & $2.59 \mathrm{E}-06$ \\
\hline
\end{tabular}

${ }^{a}$ Compounds with an asterisk have an enantiomer. 
Gibbs Free Energy of Formation of Chlordecone and Potential Degradation Products: Implications for Remediation Strategies and Environmental Fate

Table SI-3. Calculated $\Delta G^{\circ 1}$ values for reductive dechlorination of monohydrochlordecone to dihydrochlordecone ${ }^{a}$

\begin{tabular}{|c|c|c|c|c|}
\hline Reactant & $\begin{array}{l}\text { Entry in } \\
\text { Tab SI-1 }\end{array}$ & Dechlorination product & $\begin{array}{l}\text { Entry in } \\
\text { Tab SI-1 }\end{array}$ & $\begin{array}{c}\Delta G^{\circ 1} \\
\mathrm{~kJ} / \mathrm{mol}\end{array}$ \\
\hline \multirow[t]{5}{*}{ 10-monohydrochlordecone } & 2 & 6,10-dihydrochlordecone* & 16 & -161.1 \\
\hline & & cis-8,10-dihydrochlordecone* & 14 & -158.9 \\
\hline & & trans-8,10-dihydrochlodecone* & 15 & -158.1 \\
\hline & & 9,10-dihydrochlordecone* & 13 & -157.2 \\
\hline & & 10,10-dihydrochlordecone & 6 & -150.1 \\
\hline \multirow[t]{6}{*}{ 9-monohydrochlordecone } & 3 & 6,9-dihydrochlordecone & 10 & -160.2 \\
\hline & & 4,9-dihydrochlordecone & 7 & -159.3 \\
\hline & & 8,9-dihydrochlordecone* & 17 & -159.0 \\
\hline & & 9,10-dihydrochlordecone* & 13 & -156.3 \\
\hline & & 7,9-dihydrochlordecone* & 19 & -155.7 \\
\hline & & 1,9-dihydrochlordecone & 9 & -155.4 \\
\hline \multirow[t]{9}{*}{ 8-monohydrochlordecone* } & 5 & 6,7-dihydrochlordecone* & 18 & -161.1 \\
\hline & & 6,8-dihydrochlordecone* & 20 & -160.4 \\
\hline & & 3,7-dihydrochlordecone* & 21 & -159.5 \\
\hline & & 8,9-dihydrochlordecone* & 17 & -158.6 \\
\hline & & cis-8,10-dihydrochlordecone* & 14 & -157.6 \\
\hline & & trans-8,10-dihydrochlodecone* & 15 & -156.8 \\
\hline & & 7,9-dihydrochlordecone* & 19 & -155.3 \\
\hline & & 7,8-dihydrochlordecone & 11 & -153.7 \\
\hline & & 3,8-dihydrochlordecone & 8 & -142.1 \\
\hline \multirow[t]{6}{*}{ 6-monohydrochlordecone } & 4 & 6,7-dihydrochlordecone* & 18 & -165.3 \\
\hline & & 6,8-dihydrochlordecone* & 20 & -164.6 \\
\hline & & 6,10-dihydrochlordecone* & 16 & -164.0 \\
\hline & & 6,9-dihydrochlordecone & 10 & -164.0 \\
\hline & & 4,6-dihydrochlordecone & 12 & -163.9 \\
\hline & & 4,9-dihydrochlordecone & 7 & -163.1 \\
\hline
\end{tabular}

${ }^{a}$ Calculated according to the relationship: hydrochlordecone $+\mathrm{H}_{2} \rightarrow$ dihydrochlordecone $+\mathrm{H}^{+}+\mathrm{Cl}^{-} ; \Delta_{f} G^{o} \mathrm{H}^{+}$at $\mathrm{pH} 7=-39.9 \mathrm{~kJ} / \mathrm{mol} ; \Delta_{f} G^{o} \mathrm{Cl}^{-}=-131.3 \mathrm{~kJ} / \mathrm{mol}{ }^{69}$ The most favorable reactions are indicated in red.

* Compounds with an asterisk have an enantiomer that can be produced from the monohydrochlodecone indicated. The dihydro chiral compounds obtained from 8monohydrochlordecone will be in racemic mixture if the starting compound is racemic, otherwise, only one of the enantiomers will be generated. 
Gibbs Free Energy of Formation of Chlordecone and Potential Degradation Products: Implications for Remediation Strategies and Environmental Fate

Table SI-4. Change in Gibbs free energy $\left(\Delta G^{01}\right.$ in $\left.\mathrm{kJ} / \mathrm{mol}\right)$ for mineralization of chlordecone with various electron acceptors ${ }^{a}$

\begin{tabular}{lll} 
reactants & products & $\Delta G^{\text {o, }}$ \\
\hline $\mathrm{C}_{10} \mathrm{Cl}_{10} \mathrm{O}+5 \mathrm{H}_{2} \mathrm{O}+7 \mathrm{O}_{2}$ & $\rightarrow 10 \mathrm{CO}_{2}+10 \mathrm{H}^{+}+10 \mathrm{Cl}^{-}$ & -4377 \\
$\mathrm{C}_{10} \mathrm{Cl}_{10} \mathrm{O}+19 \mathrm{H}_{2} \mathrm{O}+28 \mathrm{Fe}^{3+}$ & $\rightarrow 10 \mathrm{CO}_{2}+38 \mathrm{H}^{+}+10 \mathrm{Cl}^{-}+28 \mathrm{Fe}^{2+}$ & -4252 \\
$\mathrm{C}_{10} \mathrm{Cl}_{10} \mathrm{O}+5 \mathrm{H}_{2} \mathrm{O}+14 \mathrm{NO}_{3}^{-}$ & $\rightarrow 10 \mathrm{CO}_{2}+10 \mathrm{H}^{+}+10 \mathrm{Cl}^{-}+14 \mathrm{NO}_{2}^{-}$ & -3339 \\
$5 \mathrm{C}_{10} \mathrm{Cl}_{10} \mathrm{O}+11 \mathrm{H}_{2} \mathrm{O}+28 \mathrm{NO}_{3}^{-}$ & $\rightarrow 50 \mathrm{CO}_{2}+22 \mathrm{H}^{+}+50 \mathrm{Cl}^{-}+14 \mathrm{~N}_{2}$ & -4195 \\
$2 \mathrm{C}_{10} \mathrm{Cl}_{10} \mathrm{O}+7 \mathrm{SO}_{4}^{2-}+10 \mathrm{H}_{2} \mathrm{O}$ & $\rightarrow 20 \mathrm{CO}_{2}+13 \mathrm{H}^{+}+20 \mathrm{Cl}^{-}+7 \mathrm{HS}^{-}$ & -1589 \\
$2 \mathrm{C}_{10} \mathrm{Cl}_{10} \mathrm{O}+24 \mathrm{H}_{2} \mathrm{O}$ & $\rightarrow 13 \mathrm{CO}_{2}+20 \mathrm{H}^{+}+20 \mathrm{Cl}^{-}+7 \mathrm{CH}_{4}$ & -1514 \\
\hline
\end{tabular}

${ }^{a}$ Gibbs free energy of formation data for inorganic compounds were taken from Stumm and Morgan (1996).

\section{Literature Cited}

Stumm, W.; Morgan, J. J. Aquatic Chemistry, $3^{\text {rd }}$ edition. Wiley-Interscience, New York, 1996. 
Gibbs Free Energy of Formation of Chlordecone and Potential Degradation Products: Implications for Remediation Strategies and Environmental Fate

Figure SI-1. Correlation between calculated $\Delta_{\mathrm{f}} G_{\text {gas }}^{\mathrm{o}}$ and strain energy for various classes of chlordecone congeners
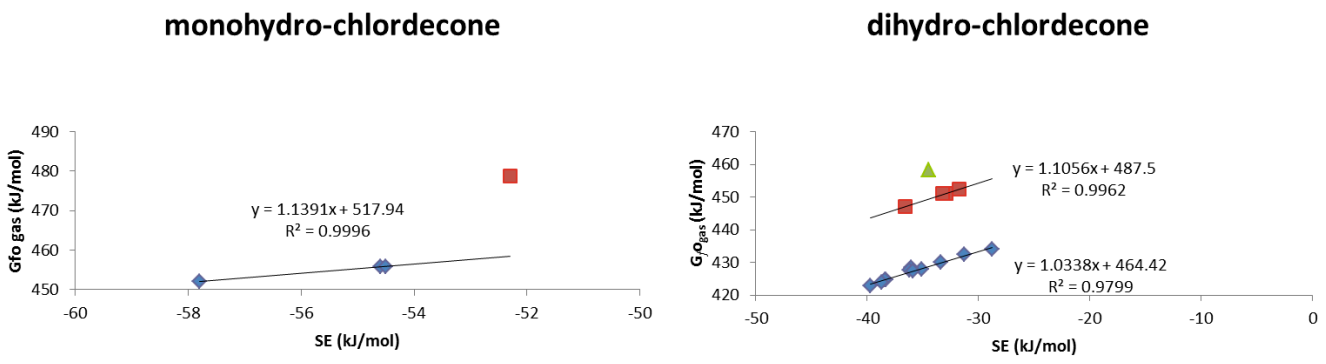

heptahydro-chlordecone

octahydro-chlordecone
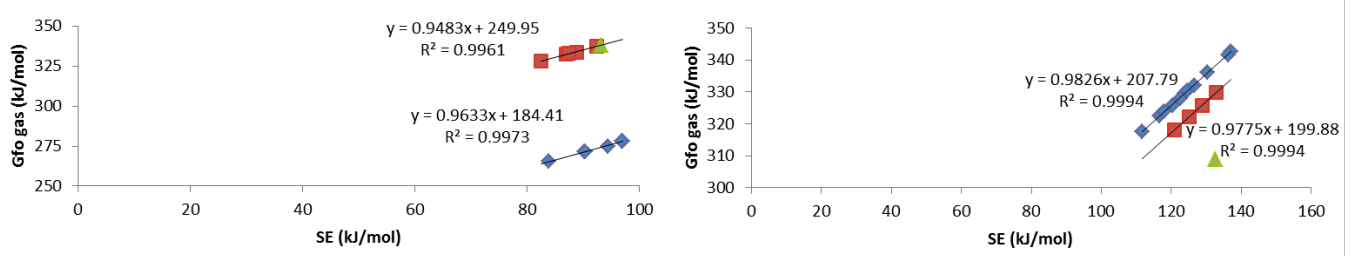

green triangles red squares blue diamonds congeners containing chlorosubstituents at both 10-positions congeners containing a chlorosubstituent at one of the 10-positions congeners without chlorosubstituents at the 10-positions 
Gibbs Free Energy of Formation of Chlordecone and Potential Degradation Products: Implications for Remediation Strategies and Environmental Fate

Figure SI-2. Correlation between calculated $\Delta_{\mathrm{f}} G_{\text {aq }}^{\mathrm{o}}$ and $\Delta_{\mathrm{f}} G^{\mathrm{o}}$ gas for the 62 chlordecone congeners listed in Table SI-2

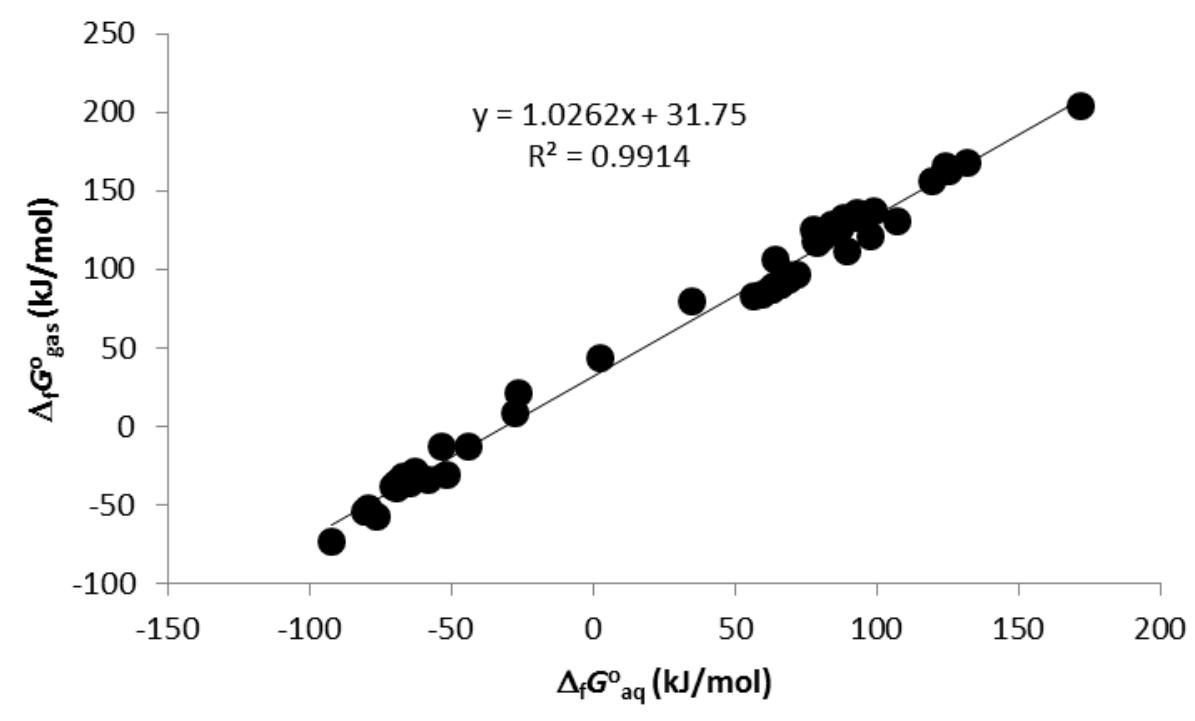


Gibbs Free Energy of Formation of Chlordecone and Potential Degradation Products: Implications for Remediation Strategies and Environmental Fate

Figure SI-3. Structure of 'chlordecone lactone' plus its corresponding standard $\Delta_{\mathrm{f}} G^{\mathrm{o}}$ and $\Delta_{\mathrm{f}} H^{\mathrm{o}}$ values in $\mathrm{kJ} / \mathrm{mol}\left(25^{\circ} \mathrm{C}\right.$ and $\left.1 \mathrm{~atm}\right)$

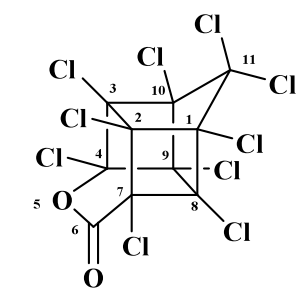

5-oxa-homochlordec-6-one (chlordecone lactone)

\begin{tabular}{lcccc} 
compound & $\boldsymbol{\Delta}_{\mathrm{f}} \boldsymbol{G}_{\text {gas }}^{\mathbf{0}}$ & $\boldsymbol{\Delta}_{\mathbf{f}} \boldsymbol{H}_{\text {gas }}^{\mathbf{0}}$ & $\boldsymbol{\Delta}_{\mathbf{f}} \boldsymbol{G}_{\mathbf{a q}}^{\mathbf{0}}$ & $\boldsymbol{\Delta}_{\mathbf{f}} \boldsymbol{H}_{\text {aq }}^{\mathbf{0}}$ \\
\hline 5-oxa-homochlordec-6-one & -222.1 & -400.0 & -239.6 & -417.8
\end{tabular}


Gibbs Free Energy of Formation of Chlordecone and Potential Degradation Products: Implications for Remediation Strategies and Environmental Fate

Figure SI-4. Estimated solubility of chlordecone congeners versus their degree of chlorination

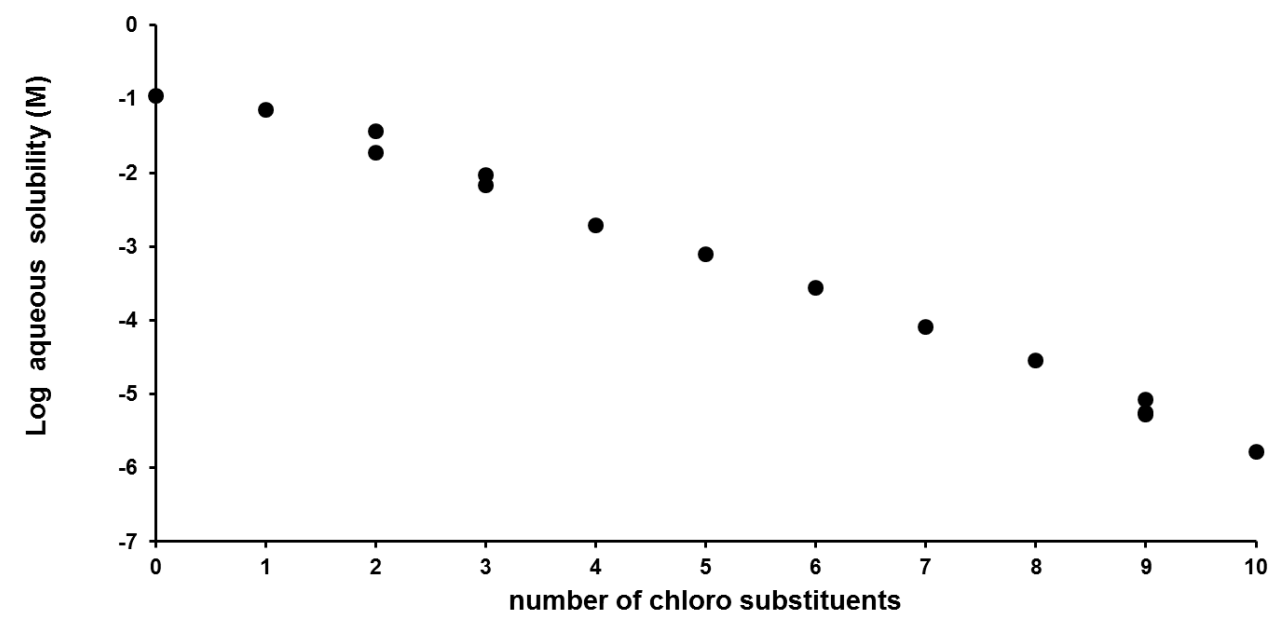

* aqueous solubility calculated with EPA's SPARC online calculator (Arp, H. P. H. et al. Environ Sci Technol 2010, 44 (12), 4400-4401; Hilal, S. H. et al. QSAR Comb Sci 2004, 23 (9), 709-720; http://sparc.chem.uga.edu/sparc/). 